\title{
Recent Progress in Photonic Processing of Metal-Oxide Transistors
}

\author{
Emre Yarali, Christina Koutsiaki, Hendrik Faber, Kornelius Tetzner, Emre Yengel, \\ Panos Patsalas, Nikolaos Kalfagiannis, * Demosthenes C. Koutsogeorgis, ** \\ and Thomas D. Anthopoulos*
}

Over the past few decades, significant progress has been made in the field of photonic processing of electronic materials using a variety of light sources. Several of these technologies have now been exploited in conjunction with emerging electronic materials as alternatives to conventional hightemperature thermal annealing, offering rapid manufacturing times and compatibility with temperature-sensitive substrate materials among other potential advantages. Herein, recent advances in photonic processing paradigms of metal-oxide thin-film transistors (TFTs) are presented with particular emphasis on the use of various light source technologies for the photochemical and thermochemical conversion of precursor materials or postdeposition treatment of metal oxides and their application in thin-film electronics. The pros and cons of the different technologies are discussed in light of recent developments and prospective research in the field of modern large-area electronics is highlighted.

\section{Introduction}

Metal oxides have emerged as promising material candidates in various electronic and optoelectronic applications. ${ }^{[1-6]}$ In comparison with amorphous silicon (a-Si) and organic semiconductors, metal oxides offer unique advantages such as

E. Yarali, Dr. H. Faber, Dr. E. Yengel, Prof. T. D. Anthopoulos King Abdullah University of Science and Technology (KAUST)

KAUST Solar Center (KSC)

Thuwal 23955, Saudi Arabia

E-mail: thomas.anthopoulos@kaust.edu.sa

C. Koutsiaki, Dr. N. Kalfagiannis, Prof. D. C. Koutsogeorgis

School of Science and Technology

Nottingham Trent University

NG11 8NS, Nottingham, UK

E-mail: nikolaos.kalfagiannis@ntu.ac.uk;

demosthenes.koutsogeorgis@ntu.ac.uk

Dr. K. Tetzner

Ferdinand-Braun-Institut

Leibniz-Institut für Höchstfrequenztechnik

Gustav-Kirchhoff-Straße 4, 12489 Berlin, Germany

Prof. P. Patsalas

Department of Physics

Aristotle University of Thessaloniki

GR-54124 Thessaloniki, Greece

The ORCID identification number(s) for the author(s) of this article can be found under https://doi.org/10.1002/adfm.201906022.

DOI: 10.1002/adfm.201906022 high mobility $(\mu)$ (even in amorphous phase), wide bandgap (transparent in the visible range), and the ability to be controllably doped. Importantly, they can be grown into thin films and various nanostructures with different scalable deposition techniques, including vacuum-based methods such as physical vapor deposition $(\mathrm{PVD})^{[7,8]}$ and chemical vapor deposition $(\mathrm{CVD})^{[9]}$ as well as solution-based processes such as spray ${ }^{[10]}$ and spin coating. ${ }^{[11]}$ Moreover, the resulting layers can be easily patterned using standard fabrication procedures and as such can be integrated into state-of-the-art processes for (opto)electronic applications. The above-mentioned capabilities have led to a plethora of applications such as switching backplanes for displays, transparent and flexible electronics, integrated circuits (ICs), photovoltaics (PVs), organic light-emitting diodes (OLEDs), capacitors, batteries, photocatalytic devices, electrochromics and memory devices, to name but a few. ${ }^{[8,12-14]}$ Because of their ability to be doped, their electronic properties can be tuned from dielectrics to semiconductors and conductors. This characteristic versatility has recently been exploited to stretch the range of their applications to new technological sectors, such as plasmonics in the near infrared and midinfrared spectral ranges..$^{[12,15]}$

One of the driving applications of metal oxides is in thinfilm transistors (TFTs) for large area electronics such as current driven optical displays and ICs. Following the early demonstrations, ${ }^{[16]}$ most effort focused on the fabrication and processing of metal oxides TFTs paying particular attention to the device performance and applications. ${ }^{[1,5,6,17]}$ Especially when processed over large areas, as in the case for display applications, the complexity to precisely control the device reliability and reproducibility becomes a challenging aspect of any TFT technology. To that respect, solution-based techniques progressed rapidly due to their lower cost and higher throughput compared to vacuum-based techniques. In both cases, the metal-oxide deposition has so far been limited to high processing temperatures $\left(>250{ }^{\circ} \mathrm{C}\right)$ (Figure 1a) which renders the technology incompatible with inexpensive, temperature-sensitive substrates such as polymers, the material class of choice for various high throughput manufacturing techniques such as roll-to-roll (R2R) and sheet-to-sheet (S2S) 
(a)

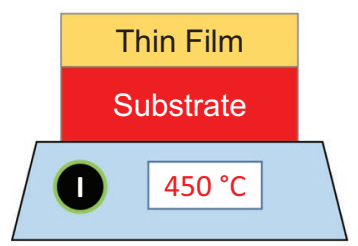

(c)

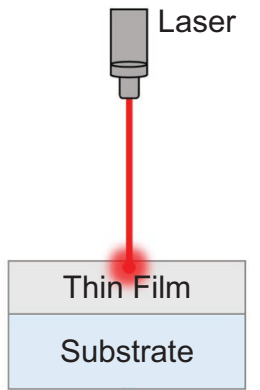

(b)

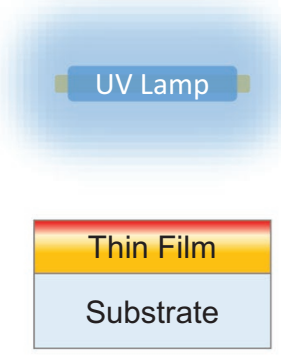

(d)

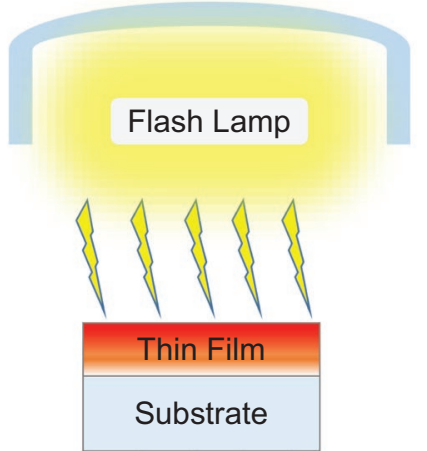

Figure 1. Schematic drawings representing: a) conventional thermal annealing (TA), b) photochemical reaction induced by continuous exposure to deep UV light, c) laser annealing, and d) flash lamp annealing.

printing. ${ }^{[2,4,18-20]} \mathrm{R} 2 \mathrm{R}$ and $\mathrm{S} 2 \mathrm{~S}$ printing are very sensitive to the thermal properties of the polymer substrates, such as polyethylene terephthalate (PET), polyethylene naphthalate (PEN), biaxially oriented polypropylene (BOPP), polycarbonate (PC), and polyphenylene sulfide (PPS), which exhibit melting points of $260,270,171,288$, and $280{ }^{\circ} \mathrm{C}$ and glass transition temperatures $\left(T_{\mathrm{g}}\right)$ of $81,120,<0,147$, and $95{ }^{\circ} \mathrm{C}$, respectively. Thus, thermal annealing at reduced temperatures has been proposed, focusing on TFTs' fabrication on polymer substrates. However, prolonged processing times (tens of minutes to hours) are typically required, which is not compatible with R2R printing, and the TFTs performance and stability were often found to be inadequate for practical applications. ${ }^{[2,4,18-20]}$

For the aforementioned reasons, the reduction of the processing temperature and time for the deposition of high-quality metal-oxide layers became the focal point of tremendous research effort in industry and academia. Consequently, new processing paradigms such as postdeposition treatments, ${ }^{[21]}$ combustion synthesis, ${ }^{[22]}$ aqueous synthesis routes, ${ }^{[23]}$ and microwave annealing, ${ }^{[24]}$ among others, have been proposed and demonstrated. However, to date, no commonly accepted standard method to (post)process metal-oxide materials on flexible substrates has been identified. Despite the significant progress, the required processing temperatures still remain significantly higher than those often encountered during photonic processing. Additionally, the processing times associated with thermal annealing are significantly longer and range from minutes to hours. To this end, photonic processing presents a viable solution to increase the speed of fabrication and overall production throughput. In terms of processing scalability of metal oxides, other alternative (post)processing techniques, such as

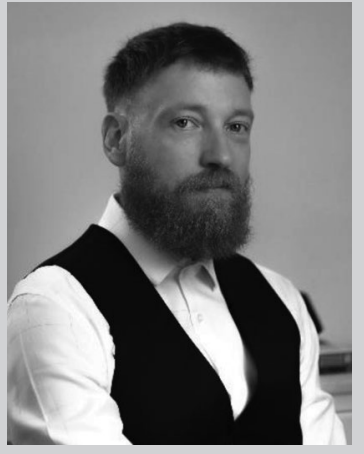

Nikolaos Kalfagiannis is a senior independent research fellow in the Physics Department of the School of Science and Technology at Nottingham Trent University (UK) and a visiting faculty of the Computational Materials Science Lab at the University of loannina (Greece). He completed his Ph.D. in nanotechnology (2009) at the Aristotle University of Thessaloniki (Greece). In 2013, he was awarded a Marie Curie Fellowship to explore the selfassembly of noble metal layers into nanoparticles using laser processing. His research focuses in the experimental and computational investigations of light-matter interactions with emphasis in laser annealing of materials and plasmonic effects.

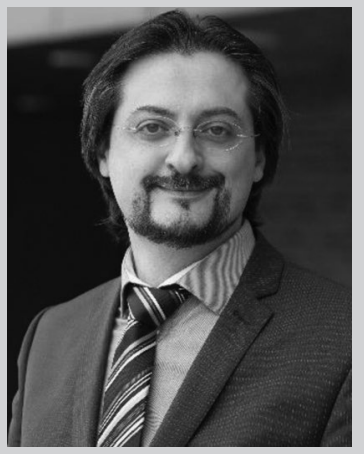

Demosthenes C. Koutsogeorgis is an associate professor of photonic technologies in the Physics Department of the School of Science and Technology at Nottingham Trent University, UK. He received his B.Sc. in physics from the University of loannina (Greece) in 1997 and his Ph.D. from the Nottingham Trent University in 2004. He has over 20 years of experience in R\&D projects, with academia and industry, on laser processing of thin-film materials for use in applications like light-emitting devices, plasmonics, and flexible electronics.

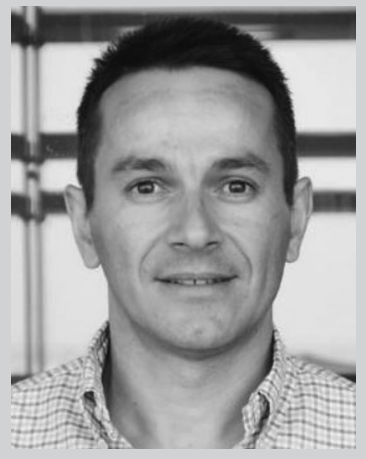

Thomas D. Anthopoulos is a professor of material science and engineering at King Abdullah University of Science and Technology (KAUST) in Saudi Arabia. He received his B.Eng. and D.Phil. degrees from Staffordshire University in the UK followed by postdoctoral appointments at the University of St. Andrews (UK) and Philips Research Laboratories (The Netherlands) before joining Imperial College London (UK) from 2006 to 2017. His research interests are diverse and cover the development and application of innovative processing paradigms and the physics, chemistry, and application of functional materials. 
microwave annealing, appear difficult to incorporate in R2R or S2S industrial settings. As the complexity of electronic circuits and systems increases, local heating and the ability to directly pattern the materials also become challenging, making the use of conventional annealing techniques even less attractive. Here, photonic processes offer potential solutions via direct and precise interaction between photons and the metal-oxide layers under processing.

Thermal annealing and direct conversion methods that rely on photonic processes have been suggested (Figure 1b-d). ${ }^{[18,25,26]}$ For those, the energy of light contained within either a narrow or broad spectrum is utilized for the fabrication of metal-oxide materials, especially on temperature sensitive substrates such as polymers. The direct interaction of light with precursor films allows for the metal-oxide synthesis/formation (particularly in the case of photochemically induced precursor reaction) and stoichiometric modification of the converted layer without the need of high thermal budget (Figure 1a). To this end, light-matter interactions offer precise energy delivery close to the surface and control over the physicochemical processes, leading to temperature gradients throughout the entire structure, which allows the substrate material to remain intact and at significantly lower temperatures.

In the case of solution-processed metal-oxide precursor layers, photochemical reactions with the deep ultraviolet (DUV) part of the electromagnetic spectrum can either offer photoinduced chemical conversion to the stable metal-oxide phase or be used as an auxiliary tool in combination with thermal annealing at relatively low temperatures (Figure 1b). ${ }^{[18]}$ Introduction of UV absorbing compounds into the precursor formulation accelerates its decomposition through the formation of radical groups that react with impurities and form the final metal-oxide framework. The continuous UV illumination can be realized using relatively simple and inexpensive setups, which compensates for the longer processing times required compared to photonic processing techniques, such as laser annealing (LA). Additionally, UV exposure through a mask enables direct patterning of the metal-oxide layers. ${ }^{[27]}$

Amongst the demonstrated photonic processing methodologies, LA has been proven to be simple and versatile, providing freedom of design, fast processing while offering compatibility with large scale manufacturing and inexpensive flexible substrates (Figure 1c). ${ }^{[28]}$ In LA, the monochromatic intense light beam is utilized in order to induce localized heating (in space as low as a few micrometers, and in time of the order of a few $\mathrm{ns}$ ) enabling control over the composition and properties of the metal oxides. A variety of process parameters can be used to tailor the laser-oxide interactions and they are discussed in detail in the upcoming sections.

In addition to LA and DUV techniques, flash lamp annealing (FLA) represents yet another widely used photonic processing method (Figure 1d). FLA relies on the use of a flash lamp (e.g., xenon) that emits photons over a broad spectrum from the UV to the near infrared (NIR) range, i.e., 200-1100 nm. During flashing, photons with different wavelengths interact with the absorptive layer, which is capable to deliver thermal energy within $\mu$ s to $\mathrm{ms}$ time range. As a result, high temperatures can be reached momentarily without subjecting the substrate to high thermal load due to the mismatch between the thermal properties of the substrate and the absorber layer. ${ }^{[29]}$ The unique combination of short processing time and unmatched scalability makes FLA a promising new technique for processing of future's large-area electronics based on metal oxides. The primary aim of this review article is to present the working principles of each photonic processing technique, provide a critical analysis of their advantages as well disadvantages but also summarize recent developments in the field of metal-oxide transistors.

\section{Photochemical Conversion of Metal-Oxide Precursors via Deep UV Illumination}

This section covers the application of continuous UV light illumination with the intent to create or improve the properties of functional materials for application in metal-oxide electronics. We will focus on the preparation of semiconducting layers based on solution-processed precursors, where the chemical conversion is either induced or complemented by the UV irradiation. Other uses include the postdeposition UV treatment of nanoparticles or vacuum deposited films aiming toward improving their physical properties and ultimately device performance. In either case, a reduction in the process temperature, as compared to conventional thermal annealing, is often observed making the UV technique viable for lowtemperature fabrication even on inexpensive and temperature sensitive substrates such as polymers.

Next, we introduce the types of UV lamps that are used, discuss the broad physical requirements for precursor formulations followed by the material classes that can be grown including, but not limited to, dielectrics, semiconductors, and conductors. Particular emphasis is placed on the implications of process parameter variations such as illumination time and process atmosphere. The potential for direct photopatterning through UV illumination of the metal-oxide materials via appropriate masking and its application in device and circuit fabrication will be discussed. Finally, a summary of the state-ofthe-art of UV-treated TFTs will be presented.

\subsection{Types of UV Light Sources}

There is a plethora of light sources that can be utilized to produce UV light and include; gas discharge lamps based on mercury, ${ }^{[23,30,31]}$ metal halides, ${ }^{[32,33]}$ deuterium $^{[34]}$ as well as various excited dimers (excimers). ${ }^{[35]}$ Other solid-state light sources such as inorganic light-emitting diodes (LEDs) are also becoming commercially available, but they typically emit at longer wavelengths than those required to initiate the photochemical reactions that form the targeted metal-oxygen-metal $(\mathrm{M}-\mathrm{O}-\mathrm{M})$ bonds from a precursor state. So far, there are no studies where semiconducting metal-oxide devices, such as TFTs, were fabricated with the aid of a UV LED.

The most frequently used type of UV lamps is that of mercury-based lamps operated at low or high pressure, with the former being the most common one. Low-pressure mercury lamps feature strong and clearly defined emission peaks 
Table 1. Emission wavelengths of selected mercury and excimer lamps. Adapted with permission. ${ }^{[35]}$ Copyright 2001, Elsevier.

\begin{tabular}{lccc}
\hline Mercury lamps & Wavelength [nm] & Excimer lamps & Wavelength $[\mathrm{nm}]$ \\
\hline Low pressure & 185 and 254 & $\mathrm{NeF}^{*}$ & 108 \\
& & $\mathrm{Ar}_{2}{ }^{*}$ & 126 \\
Medium pressure & \multirow{2}{*}{$200-600$} & $\mathrm{Xe}_{2}{ }^{*}$ & 172 \\
& & $\mathrm{KrCl}^{* *}$ & 222 \\
High pressure & \multirow{3}{*}{$300-600$} & $\mathrm{KrF}^{*}$ & 248 \\
& & $\mathrm{Br}_{2}{ }^{*}$ & 289 \\
& & $\mathrm{XeF}$ & 351 \\
\hline
\end{tabular}

at $253.7 \mathrm{~nm}$ (90\% of emission power) and $184.9 \mathrm{~nm} \mathrm{(10 \% ).}$ The energies at these wavelengths (472 and $647 \mathrm{~kJ} \mathrm{~mol}^{-1}$ ) are adequate to break the bonds of common chemical groups such $\mathrm{C}-\mathrm{O}, \mathrm{C}-\mathrm{C}$ and $\mathrm{C}-\mathrm{H}$ which are found in many soluble metaloxide precursors. ${ }^{[34,36]}$ Additionally, the shorter wavelength photons promote the formation of ozone $\left(\mathrm{O}_{3}\right)$ when the DUV treatment is carried out in an air environment, which results in further chemical reactions. ${ }^{[36-39]}$

Another type of UV lamp uses excimers of noble gases or noble gases/halide mixtures that are created by a dielectric barrier discharge. Depending on the gas molecules, the radiative excimer decomposition results in narrow emission bands of high intensity at a particular wavelength, i.e., $\lambda=126 \mathrm{~nm}$ for $\mathrm{Ar}_{2}{ }^{*}$ or $\lambda=172 \mathrm{~nm}$ for $\mathrm{Xe}_{2}{ }^{*}$. As can be seen in Table 1, the peak emission of such lamps can be tuned between $\approx 108$ and $351 \mathrm{~nm} \cdot{ }^{[35]}$ This variability allows one to select a wavelength that is suitable for a given photochemical process or (precursor) material.

The emission spectrum of the lamp used is an important parameter for the efficient photoconversion of a given metal-oxide precursor. Apart from the most commonly used low pressure mercury lamps, high-pressure mercury lamps with an emission peak $\left(\lambda_{\max }\right)$ at $365 \mathrm{~nm},{ }^{[40]}$ deuterium lamps $\left(\lambda_{\max }=250 \mathrm{~nm}\right)^{[41]}$ and metal halide lamps $\left(\lambda_{\max }=370 \mathrm{~nm}\right)^{[32]}$ are also often employed for photochemical conversion. Besides, excimer sources give a wide range of emission spectra that depends on the choice of the radiative excimer. ${ }^{[35]}$ Along with the spectrum of the lamp, the efficiency of the photoinduced conversion depends on various additional parameters, one of which is the emitted power density. The levels of irradiance (optical power per unit area) reported in the literature are rather wide and spans from $0.8 \mathrm{~mW} \mathrm{~cm}{ }^{-2}$ (lower end) ${ }^{[42]}$ up to $1 \mathrm{~W} \mathrm{~cm}^{-2}$ (higher end). ${ }^{[32]}$ Many studies, however, used DUV lamps with irradiance values smaller than $60 \mathrm{~mW} \mathrm{~cm}^{-2}[34,39,43-49]$ On the other hand, the output optical power density can be significantly affected by the geometry of the setup and particularly by the distance between the UV source and the sample. ${ }^{[3]}$ Similarly, the duration of the illumination process is also critical and is often found to vary between a few minutes up to $12 \mathrm{~h},{ }^{[48-52]}$ although times between $15-30 \mathrm{~min}$ are the most frequently used. ${ }^{[41,47,51,53,54]}$ Finally, the oxygen content and relative humidity $(\mathrm{RH})$ in ambient air are also critical parameters when photonic processing is performed in air. For this reason, DUV treatment of metal oxides has been studied in different ambient conditions, including air, vacuum and $\mathrm{N}_{2}$ with varying humidity levels. ${ }^{[55]}$

\subsection{Photochemical Conversion Process, Materials, and Precursors}

The main purpose of exposing a metal-oxide precursor to DUV light is to either initiate, perform, or aid its conversion to the stoichiometric stable metal-oxide phase. First and foremost, in order for such a photochemical conversion process to be efficient, the precursor itself needs to absorb at least a part of the emitted spectrum. This can be either due to the inherent absorption properties of the precursor material and solvent combination or induced through the addition of suitable photoactive components. For example, the use of different metal salts incorporating the same metal but different anions exhibit different light absorption characteristics. As shown in Figure 2a, the solution of indium chloride in 2-methoxyethanol (2ME) shows a considerably weaker absorption compared to the nitrate counterpart, making indium nitrate a more suitable candidate for a photochemical activated conversion process. On the other hand, UV-absorbing compounds such as betadiketones benzoylacetone (BzAc) or acetylacetone (AcAc), are commonly used photosensitizers known to aid photochemical conversion. $^{[43,56-59]}$

The addition of photosensitizer components is known to form chelate bonds with metal ions, which is the origin for the increased absorption (Figure 2b). Upon UV irradiation, the metal-chelate bonds in the precursor film are gradually decomposing, giving rise to hydrolysis and condensation reactions that eventually leads to the formation of an $\mathrm{M}-\mathrm{O}-\mathrm{M}$ framework. In their seminal paper in 2012, Kim et al. described for the first time the photochemical conversion of nitrate-based sol-gel precursors via DUV irradiation to form semiconducting metal oxides (e.g., indium gallium zinc oxide (IGZO), indium zinc oxide (IZO), and $\mathrm{In}_{2} \mathrm{O}_{3}$ ) that were subsequently incorporated into metal-oxide TFTs. ${ }^{[18]}$ They described the conversion as a two-step process, where an initially rapid photochemical condensation was followed by more gradual densification accompanied by structural rearrangements and removal of remaining impurities (Figure $2 \mathrm{c}$ ). It was found that during the process, the substrate was unintentionally heated up to $150^{\circ} \mathrm{C}$. This increased temperature was vital for the successful metaloxide formation as volatile organic residues could be removed more effectively. When substrates were artificially cooled down during DUV illumination, or when films were only thermally annealed at $150{ }^{\circ} \mathrm{C}$ without illumination, no functioning devices were obtained. Thus, the combination of moderate thermal annealing and DUV treatment proved to be necessary for the synthesis of dense, high-quality metal oxides. Films converted this way were shown to exhibit comparable properties to reference oxide layers synthesized via thermal annealing at $350{ }^{\circ} \mathrm{C}$.

In a separate study, the same group investigated the photoconversion of various dielectric materials, including $\mathrm{AlO}_{x}$, $\mathrm{ZrO}_{x}$, and $\mathrm{HfO}_{x} \cdot{ }^{[51]}$ In this study, Park et al. proposed that DUV light induced photolysis of metal ligands (e.g., nitrate, acetylacetonate, isopropoxide) creates radicals which in turn help to remove residual organic impurities as well as accelerate the layer densification process. The latter was monitored by the reduction in film thickness of as-spun films as thermal annealing progressed with or without simultaneous illumination. The thermally assisted photoactivation led to faster and 

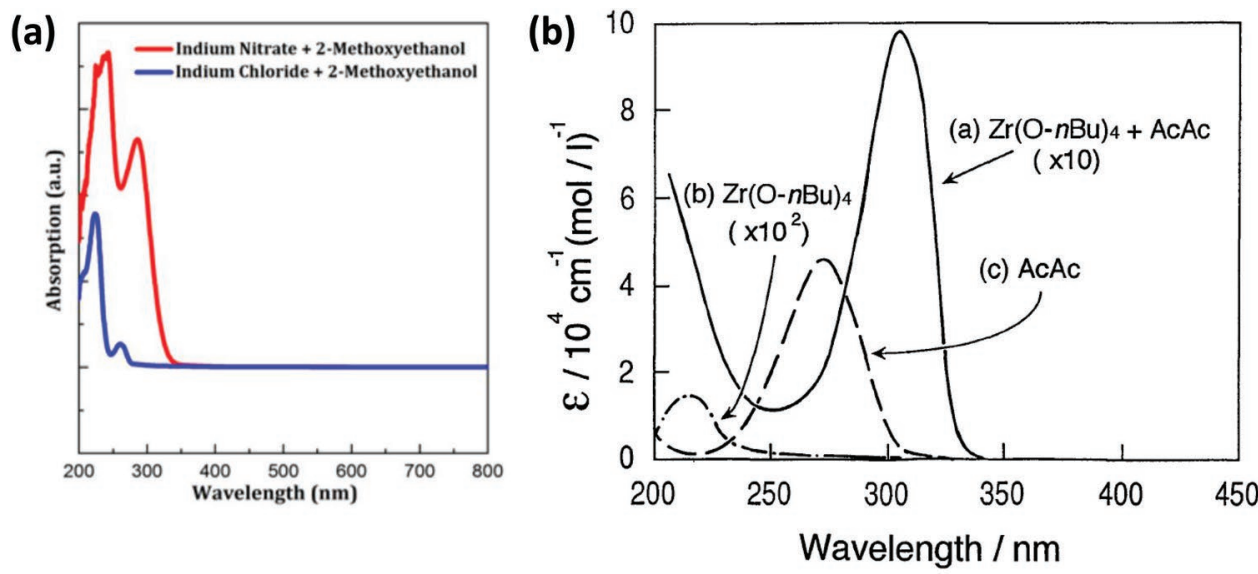

(c)

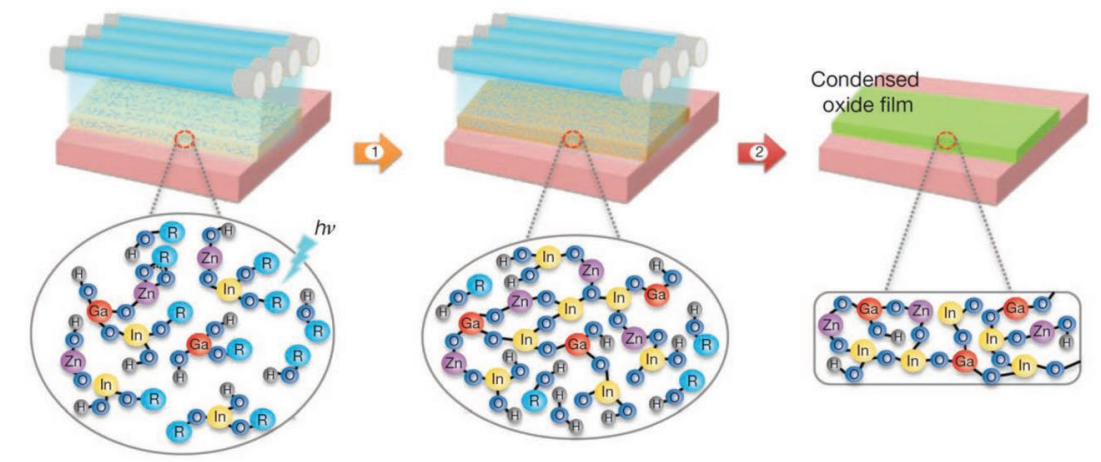

Figure 2. a) UV light absorption spectra of indium nitrate versus indium chloride. Reproduced with permission. ${ }^{[48]}$ Copyright 2016, American Chemical Society. b) Increased UV light absorption through the addition of acetylacetone to Zr-n-butoxide precursor. Reproduced with permission. ${ }^{\left[{ }^{7]}\right]}$ Copyright 1994, The Japan Society of Applied Physics. c) Schematic depiction of DUV photochemical conversion process. Reproduced with permission. ${ }^{[18]}$ Copyright 2012, Springer Nature.

improved layer densification. Oxide layers processed via simultaneous DUV illumination and thermal annealing at $150{ }^{\circ} \mathrm{C}$ produced dense films that were comparable in quality to those prepared by thermal annealing at $350{ }^{\circ} \mathrm{C}$.

Worth noting is that the aforementioned studies were carried out in a nitrogen atmosphere since in air, UV light is absorbed by oxygen which in turn creates ozone. As such, the amount of UV light involved in the photochemical conversion decreased drastically resulting in devices with poor operating characteristics. ${ }^{[18]}$ However, choosing air as the process atmosphere for a DUV treatment can have beneficial effect as well. Umeda et al. studied the effect of DUV irradiation alone without ozone ( $\mathrm{N}_{2}$ environment), ozone without DUV (using a separate ozonizer) and the combination of both (DUV in ambient air) before thermal annealing (TA). ${ }^{[36]}$ They concluded that high energy photons are responsible for the decomposition of relevant organic ligands (e.g., $\mathrm{C}=\mathrm{O}$ and $\mathrm{C}-\mathrm{H}$ bonds) but that the presence of $\mathrm{O}_{3}$ is critical in removing the decomposed elements. An application of $\mathrm{DUV} / \mathrm{O}_{3}$ treatment prior to thermal annealing of IGZO precursor films led to an increased amount of $\mathrm{M}-\mathrm{O}$ bonds in the final material and a significantly improved performance of the resulting TFTs when compared to devices prepared via conventional thermal annealing.

With the aim of improving film quality and device performance further, Kim et al. combined DUV with appropriate precursor combustion chemistry. ${ }^{[22]}$ In this approach, part of the energy required for the precursor conversion is generated insitu from the interaction between an added fuel and the oxidizer component. Oxidizers are typically nitrate groups, whereas efficient fuel components are acetylacetone or urea. As a result, the annealing temperature of these engineered precursor formulations can be drastically reduced as compared to conventional precursors. DUV irradiation was found to be an efficient way to initiate the combustion reaction and was used extensively for the synthesis of a variety of metal oxides. ${ }^{[27,41,60-62]}$ To this end, Rim et al. found that device performance could be increased significantly by adding $\mathrm{NH}_{4} \mathrm{OH}$ in the precursor, which acted both as a chelating agent as well as another oxidizer. ${ }^{[27]} \mathrm{A}$ possible downside of combustion synthesis, pointed out by Daunis et al., comes into play when higher film thicknesses are desired, e.g., for dielectric layers. ${ }^{[61]}$ The rapid heat evolution and the corresponding release of gaseous reaction by-products in thick precursor films can lead to increased levels of porosity. Therefore, the authors opted to use combustion synthesis only to process thin $\mathrm{In}_{2} \mathrm{O}_{3}$ semiconductor layers but not for the growth of thicker $\mathrm{AlO}_{x}$ dielectrics. While the precursors in most of the aforementioned studies were based on organic solvents (e.g., 2ME), Heo et al. obtained slightly improved device performance for IGZO TFTs when deionized water was used as the solvent instead of $2 \mathrm{ME}{ }^{[63]}$ The reduced concentration of organic residues found in the formed IGZO film was seen as the cause for the improvement. 
Overall, for the fabrication of metal-oxide electronic devices such as thin-film transistors, there is a wide range of precursors to choose from. The most common choices include metal compounds based on nitrates, ${ }^{[2,27,34,40,41,43,47,48,51,55,61-66]}$ acetylacetonates, ${ }^{[23,30,33,37,65-69]}$ alkoxides, ${ }^{[36,44,51]}$ or acetates ${ }^{[18,38,43,63,66]}$ and to a lesser extent chlorides, ${ }^{[43,62]}$ acrylates, ${ }^{[53]}$ and hydroxides. ${ }^{[23,49]}$ The kinds of materials that were successfully processed with the help of DUV irradiation include all those that are required to form metal-oxide TFTs, i.e., semiconductors, dielectrics, and conductors. Among them, the most commonly employed n-type semiconductors are IGZO, ${ }^{18,27,36,38,47,63,66,70,71]}$ IZO, ${ }^{[18,34,44,48,55,65,68,72]}$ zinc tin oxide (ZTO), ${ }^{[72]}$ as well as the binary compounds $\mathrm{ZnO},[23,49,53] \quad \operatorname{In}_{2} \mathrm{O}_{3},{ }^{[18,34,37,40,41,48,51,54,61,64]}$ and $\mathrm{GaO}_{x} \cdot{ }^{\left[{ }^{64]}\right]}$ For p-type oxides, there are far fewer studies available, with $\mathrm{Cu}_{2} \mathrm{O}$ being one of the exceptions. ${ }^{[73]}$

Regarding dielectric materials, the most prominent examples are found to be $\mathrm{Al}_{2} \mathrm{O}_{3}{ }^{[23,27,32,41,51,61,62]}$ and $\mathrm{ZrO}_{x}[23,30,33,37,51,67,69]$ and their derivatives $\left(\mathrm{ZrAlO}_{x}^{[66]}\right.$ or $\left.\mathrm{YAlO}_{x}{ }^{[74]}\right)$ as well as $\mathrm{HfO}_{x}{ }^{[51,62]}$ and $\mathrm{SiO}_{2} \cdot{ }^{[75]}$ On the other hand, the pool of conductive materials mostly contains indiumdoped tin oxide (ITO), ${ }^{[27,43]}$ antimony doped $\mathrm{SnO}_{2}{ }^{[58]}$ or nanoparticle layers based on Ag. ${ }^{[76]}$ Irrespective of the type of the metal oxide, however, one of the main motivations for using photonic processing is the reduction of the thermal budget, which is crucial for the fabrication of TFTs and circuits on temperature sensitive substrate materials such as plastics including, polyimide (PI), ${ }^{[27,47,51,63,66]} \mathrm{PEN}^{[23,67]}$ polyarylate, ${ }^{[18]}$ and PET, ${ }^{[33]}$ as well as more specialized systems such as shape memory polymers. ${ }^{[61]}$

\subsection{Process Variations and the Impact on Material and Device Operation}

DUV treatment can be applied at different stages during device processing. For example, DUV irradiation has been utilized in combination with solution-processing either before thermal annealing (TA), simultaneously with TA, or on its own. In the case of vacuum deposited metal-oxide films, the DUV treatment often takes the form of a postdeposition step with ${ }^{[39]}$ or without $^{[77,78]}$ simultaneous TA. Furthermore, the application of DUV light can be carried out across a wide range of processing times depending on the application and the type of UV lamp employed. It has been shown that exposure for only $30 \mathrm{~s}$ is sufficient when used as a mean for patterning the metal-oxide precursor, ${ }^{[42]}$ but increases to tens of minutes, or even several hours, when a complete chemical conversion is required. ${ }^{[23,52]}$ An overview of the electrical properties of selected DUV treated metal-oxide TFTs can be found in Table 2.

\subsubsection{Application of DUV before Thermal Annealing}

Performing a DUV light treatment step on a metal-oxide precursor layer prior to thermal annealing can serve two purposes. First, irradiation can initiate photochemical conversion which in turn renders exposed parts of the films insoluble to a subsequent development step while retaining the solubility of unexposed areas. Second, DUV irradiation prior to annealing has been shown to play the role of an auxiliary step that helps

Table 2. Selected publications on DUV treated metal-oxide TFTs and their electrical properties. The following abbreviations are used: SC, semiconductor; $G D$, gate dielectric; $G$, gate; S/D, source/drain; RT, room temperature; NG, not given; uh, unintentional heating.

\begin{tabular}{|c|c|c|c|c|c|c|c|c|c|c|c|}
\hline $\begin{array}{l}\text { DUV treated layer/ } \\
\text { duration/ambient }\end{array}$ & Semiconductor & Dielectric & Process & Substrate & $\begin{array}{c}\text { Power } \\
\text { density } \\
{\left[\mathrm{mW} \mathrm{cm}{ }^{-2}\right]}\end{array}$ & $\begin{array}{c}\text { Carrier } \\
\text { mobility } \\
{\left[\mathrm{cm}^{2} \mathrm{~V}^{-1} \mathrm{~s}^{-1}\right]}\end{array}$ & $I_{\mathrm{ON}} / I_{\mathrm{OFF}}$ & $\begin{array}{l}\text { Sub-threshold } \\
\text { slope (SS) } \\
{\left[\mathrm{V} \mathrm{dec}^{-1}\right]}\end{array}$ & $\begin{array}{c}\text { Max. process } \\
\text { temperature } \\
{\left[{ }^{\circ} \mathrm{C}\right]} \\
\end{array}$ & Ref. & Year \\
\hline GD/90 min/air & $\begin{array}{c}\text { Organic SC } \\
\text { (PBTTT-C 14) }\end{array}$ & ZrOx (6-7 nm) & Spin coating & $\mathrm{Si}$ & 270 & 0.016 & $10^{4}$ & 0.15 & RT & [30] & 2011 \\
\hline $\mathrm{SC} / 120 \mathrm{~min} / \mathrm{N}_{2}$ & IGZO & $\operatorname{ALD~AlO}_{x}(35 \mathrm{~nm})$ & Spin coating & PAR & 28 & 3.7 & $\approx 10^{8}$ & 0.096 & 150 (uh) & [18] & 2012 \\
\hline $\begin{array}{l}\text { SC for patterning } \\
\text { (solubility contrast)/ } \\
10 \mathrm{~min} / \text { air }\end{array}$ & IGZO & $\mathrm{SiO}_{2}(100 \mathrm{~nm})$ & Spin coating & $\mathrm{Si} / \mathrm{SiO}_{2}$ & NG & 15.5 & $\approx 10^{8}$ & NG & $350 \mathrm{TA}$ & [27] & 2014 \\
\hline $\begin{array}{l}\mathrm{SC}, \mathrm{GD}, \mathrm{G}, \mathrm{S}-\mathrm{D} \text { for } \\
\text { patterning (solubility } \\
\text { contrast) } / 10 \mathrm{~min} / \text { air }\end{array}$ & IGZO & $\mathrm{AlO}_{x}(150 \mathrm{~nm})$ & Spin coating & $\mathrm{PI}$ & NG & 84 & $>10^{5}$ & NG & $350 \mathrm{TA}$ & [27] & 2014 \\
\hline $\mathrm{SC}$ and $\mathrm{GD} / 30 \mathrm{~min} / \mathrm{N}_{2}$ & $\ln _{2} \mathrm{O}_{3}$ & $\mathrm{AlO}_{x}(20 \mathrm{~nm})$ & Spin coating & $\mathrm{PI}$ & $25-28$ & 8.02 & $\approx 10^{8}$ & 0.203 & $<150$ & {$[51]$} & 2015 \\
\hline $\mathrm{SC}$ and $\mathrm{GD} / 120 \mathrm{~min} / \mathrm{N}_{2}$ & IGZO & $\mathrm{AlO}_{x}: \operatorname{Zr}(35 \mathrm{~nm})$ & Spin coating & $\mathrm{PI}$ & ng & 8.58 & $10^{8}-10^{9}$ & 0.153 & 150 (uh) & {$[66]$} & 2015 \\
\hline $\begin{array}{l}\text { SC, SUT at } 150^{\circ} \mathrm{C} / \\
60 \mathrm{~min} / \text { air }\end{array}$ & IGZO & $\mathrm{SiO}_{2}(120 \mathrm{~nm})$ & Sputtering & $\mathrm{Si} / \mathrm{SiO}_{2}$ & 60 & 15.81 & $10^{8}$ & 0.57 & 150 & [39] & 2016 \\
\hline $\begin{array}{l}\mathrm{GD}, \mathrm{SUT} \text { at } 150^{\circ} \mathrm{C} / \\
30 \mathrm{~min} / \mathrm{N}_{2}\end{array}$ & ICZO (sputtered) & HfOx (26 nm) & Spin coating & $\mathrm{Si}$ & 75 & 43.9 & $10^{6}$ & 0.066 & 150 & {$[62]$} & 2017 \\
\hline $\mathrm{SC} / 120 \mathrm{~min} / \mathrm{N}_{2}$ & $\ln _{2} \mathrm{O}_{3} / \mathrm{GaOx}$ & $\mathrm{SiO}_{2}(200 \mathrm{~nm})$ & Spin coating & $\mathrm{Si} / \mathrm{SiO}_{2}$ & NG & 11.2 & $\approx 10^{8}$ & 0.61 & $\begin{array}{c}\text { No intentional } \\
T A\end{array}$ & {$[64]$} & 2017 \\
\hline $\begin{array}{l}\text { Substrate for patterning } \\
\text { (wetting contrast), } \\
3 \mathrm{~min}\end{array}$ & IGZO & $\mathrm{SiO} 2$ & Bar coating & $\mathrm{Si} / \mathrm{SiO}_{2}$ & 10 & 12.78 & $\approx 10^{8}$ & 0.58 & 400 & [71] & 2017 \\
\hline$G D / 20 \mathrm{~min} / \mathrm{air}$ & $\ln _{2} \mathrm{O}_{3}$ & AlOx $(15-200 \mathrm{~nm})$ & Ink-jet & $\mathrm{Si}$ & 1000 & 12 & $>10^{7}$ & 0.15 & 250 & [32] & 2018 \\
\hline
\end{tabular}



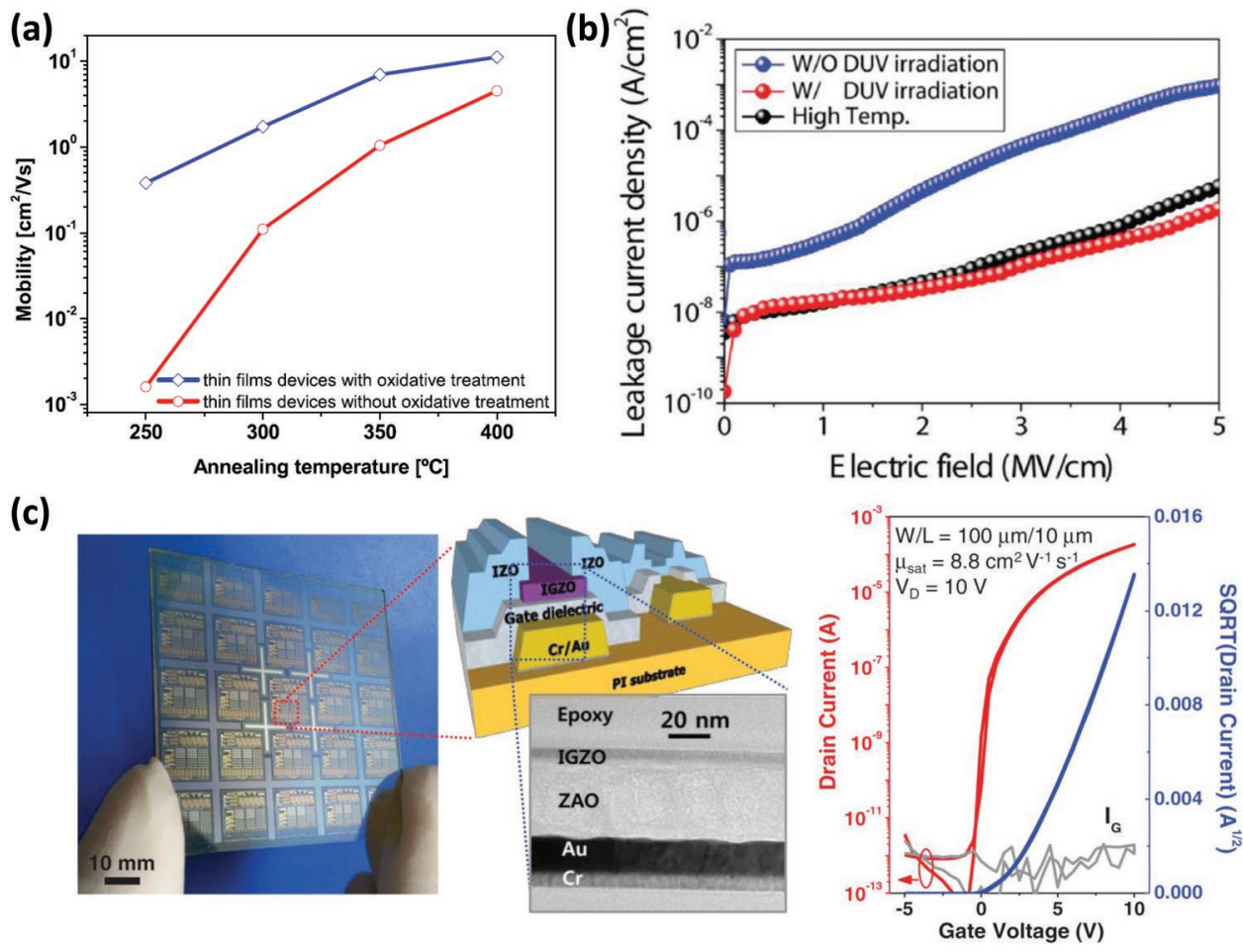

Figure 3. a) Electron mobility of solution processed IZO devices annealed at different temperatures with and without auxiliary DUV treatment in air prior to the final thermal annealing. Reproduced with permission. ${ }^{[68]}$ Copyright 2014, IEEE. b) Comparison of leakage current densities of sol-gel aluminum oxide layers. The films were either annealed at $350^{\circ} \mathrm{C}$ (High Temp.), kept at $150^{\circ} \mathrm{C}$ (w/o DUV irradiation) or processed with a simultaneous DUV exposure and TA at $150^{\circ} \mathrm{C}$ (W/DUV irradiation). Reproduced with permission. ${ }^{[51]}$ Copyright 2015, Wiley-VCH. c) An array of IGZO TFTs with zirconium aluminum oxide (ZAO) dielectric on PI substrate. Both IGZO and ZAO were processed via DUV irradiation with unintentional heating to $150^{\circ} \mathrm{C}$. Reproduced with permission. ${ }^{[66]}$ Copyright 2015, Wiley-VCH.

to improve the degree of precursor conversion while lowering the maximum TA temperature required. The latter approach has been successfully applied for the fabrication of $\operatorname{IGZO}^{[36,38,47]}$ and IZO ${ }^{[68]}$ TFTs, as well as ink-jet printed $\mathrm{AlO}_{x}$ dielectrics. ${ }^{[32]}$

A common finding of the aforementioned studies is the reduced amount of residual carbon impurities in the oxide films and an overall improvement of the device operation as compared to layers/devices prepared without the DUV step. Consequently, for a given thermal annealing temperature, the measured carrier mobility of TFTs with prior DUV treatment was found to be significantly improved as compared to devices prepared by TA alone. ${ }^{[36,68]}$ An example of this effect is shown in Figure 3a for IZO based TFTs where the carrier mobility is an order of magnitude higher for DUV treated devices. Furthermore, the bias stress stability of IGZO TFTs was found to improve with threshold voltage shifts $\left(\Delta V_{\mathrm{TH}}\right)$ of 7.5 and $1.8 \mathrm{~V}$ for transistors (measured negative bias stress for $60000 \mathrm{~s}$ ) without and with auxiliary DUV treatment, respectively. ${ }^{[38]}$ In the case of ink-jet printed $\mathrm{AlO}_{x}$ layers, Scheideler et al. reported a reduced frequency dispersion of the dielectric constant as well as an increased breakdown electric field $\left(5.1 \mathrm{MV} \mathrm{cm}^{-1}\right)$ when auxiliary DUV treatment was performed prior to TA. ${ }^{[32]}$ The same team also showed that DUV treatment allowed deposition of thicker $\mathrm{AlO}_{x}$ layer (up to $200 \mathrm{~nm}$ ) within a single deposition step, whereas $\mathrm{AlO}_{x}$ films that have not undergone DUV treatment showed the appearance of cracks when the layer thicknesses exceeded $100 \mathrm{~nm}$. By combining the $\mathrm{AlO}_{x}$ dielectric with ink-jet printed $\operatorname{In}_{2} \mathrm{O}_{3}$ films, the team demonstrated $\operatorname{In}_{2} \mathrm{O}_{3}$ TFTs with high electron mobility of $12 \mathrm{~cm}^{2} \mathrm{~V}^{-1} \mathrm{~s}^{-1}$ at a maximum process temperature of $250^{\circ} \mathrm{C}$.

All the aforementioned studies were carried out in ambient air, and thus, ozone production was concurrent during DUV illumination. As already discussed, $\mathrm{O}_{3}$ is an effective agent that promotes the removal of residual organic impurities. To this end, Ogura et al. reported TFTs with improved performance when carrying out the DUV treatment in humid air (90\% RH) which was attributed to the formation of additional reactive hydroxyl species in the process. ${ }^{[4]}$

\subsubsection{DUV Treatment Combined with Thermal Annealing}

The studies discussed in the following section focus on the simultaneous use of DUV and thermal annealing. ${ }^{[34,41,44,49,62,74]}$ While the annealing temperature is typically $<200{ }^{\circ} \mathrm{C}$, there are some exceptions where higher temperatures were reached. ${ }^{[42,55]}$ Park et al. investigated the formation of dielectric films such as $\mathrm{AlO}_{x}, \mathrm{ZrO}_{x}, \mathrm{HfO}_{x}$, via DUV exposure for $30 \mathrm{~min}$ in $\mathrm{N}_{2} \cdot{ }^{[1]} \mathrm{Com}$ pared to conventional TA performed at temperatures between 50 and $150{ }^{\circ} \mathrm{C}$, they found that the formed layers were significantly thinner when films subjected simultaneously to DUV light and high temperature TA. Similarly, the leakage current density through the oxide dielectric was found to reduce to levels only achieved via high-temperature annealing (Figure 3b). 
TFTs using solution-processed $\mathrm{AlO}_{x}$ dielectric and $\mathrm{In}_{2} \mathrm{O}_{3}$ as the channel semiconductor (same DUV treatment) could be processed on PI and showed electron mobility of $8.02 \mathrm{~cm}^{2} \mathrm{~V}^{-1} \mathrm{~s}^{-1}$ while multi-stage ring oscillators made with the TFTs operated at an oscillation frequency over $650 \mathrm{kHz}$ even when subjected to mechanical stress (bending radius $<1 \mathrm{~mm}$ ).

Carlos et al. ${ }^{[41]}$ focused on dielectric materials using the aforementioned combinatorial approach. Films of $\mathrm{AlO}_{x}$ were produced using 15-30 min exposure to UV light $\left(\lambda_{\text {peak }}=160 \mathrm{~nm}\right)$ in nitrogen at temperatures $<200{ }^{\circ} \mathrm{C}$. Combining sputtered IGZO or solution processed $\mathrm{In}_{2} \mathrm{O}_{3}$, low-voltage TFTs with electron mobility values of 6.3 and $5.57 \mathrm{~cm}^{2} \mathrm{~V}^{-1} \mathrm{~s}^{-1}$, respectively, were obtained. The device performance was improved further by growing multilayer dielectrics of $\mathrm{AlO}_{x}$ and $\mathrm{HfO}_{x}$, through a combination of DUV and TA at $150{ }^{\circ} \mathrm{C} .{ }^{[62]}$ The resulting multilayers exhibited improved characteristics yielding low operating voltage TFTs with a maximum electron mobility of $43.9 \mathrm{~cm}^{2} \mathrm{~V}^{-1} \mathrm{~s}^{-1}$. A similar approach was successfully employed to create ink-jet printed yttrium aluminum oxide dielectrics by Bolat et al. ${ }^{[74]}$

A dramatic reduction in the processing time was obtained using zinc hydroxide-based precursor formulations in aqueous ammonia. ${ }^{[23]}$ This chemical route is known for its low decomposition temperature $\left(<200{ }^{\circ} \mathrm{C}\right)$, but requires longer annealing time typically on the order of $1 \mathrm{~h}$. Hwang et al. demonstrated ZnO TFTs with mobility of $\approx 0.6 \mathrm{~cm}^{2} \mathrm{~V}^{-1} \mathrm{~s}^{-1}$ for either via TA for $1 \mathrm{~h}$ at $150^{\circ} \mathrm{C}$, or via simultaneous DUV treatment and TA $\left(150{ }^{\circ} \mathrm{C}\right)$ for only 3 min..$^{49]}$ Synthesis of semiconducting IZO films from nitrates or alkoxides precursors has also been produced through a combination of UV exposure and TA, albeit at slightly elevated temperatures $\left(200-300^{\circ} \mathrm{C}\right)$ and longer process times $(4 \mathrm{~h}) \cdot{ }^{[34,42,44,55,79]}$

\subsubsection{Photochemical Conversion via DUV Light}

In this section, we summarize relevant work where no intentional TA steps were involved during the synthesis and growth of the metal oxide. We note, however, that due to lamp design and often long duration of the DUV illumination step, the sample may undergo unintentional heating. The latter was shown in many occasions to be vital for the successful synthesis of different oxides, ${ }^{[18,48,66]}$ although it is not always measured/ reported hence making a direct comparison of the experimental procedures in different laboratories challenging.

In 2011 Park et al. ${ }^{[30]}$ reported the synthesis and use of solution-processed $\mathrm{ZrO}_{x}$ via DUV illumination. The precursor formulation of zirconium acetylacetonate in $\mathrm{N}, \mathrm{N}$-dimethylformamide was subjected to DUV illumination in air for $90 \mathrm{~min}$ without intentional heating. The formed dielectric layers were subsequently used in low-voltage transistors based on a solution-processed organic semiconductor as the channel material. In 2013, the same group extended the work and studied the formation of $\mathrm{ZrO}_{x}$ while demonstrating the compatibility of the conversion process with temperature sensitive polymer PET substrates. ${ }^{[33]}$ Following, $\mathrm{Xu}$ et al. fabricated relatively thick (22 nm) $\mathrm{ZrO}_{x}$ films with a somewhat unexpectedly high breakdown electric field (18 $\mathrm{MV} \mathrm{cm}^{-1}$ ) and combined them with solution-processed $\mathrm{ZnO}$ to realize TFTs at temperatures below $150{ }^{\circ} \mathrm{C} .{ }^{[69]} \mathrm{A}$ precursor formulation composed of $\mathrm{ZnO}$ hydrate in ammonium hydroxide was used by Lin et al. in combination with DUV to grow ultrathin (4-6 nm) ZnO layers atop a solution processed bilayer $\mathrm{AlO}_{x} / \mathrm{ZrO}_{x}$ dielectric. ${ }^{[23]}$ Despite the low processing temperature $\left(<180^{\circ} \mathrm{C}\right)$, the resulting TFTs exhibited mobility values of 11 and $4-5 \mathrm{~cm}^{2} \mathrm{~V}^{-1} \mathrm{~s}^{-1}$ when fabricated on glass and PEN substrates, respectively. Going a step further, the same team combined DUV and low temperature TA for the growth of bilayer dielectrics (e.g., $\mathrm{AlO}_{x} / \mathrm{ZrO}_{x}$ ) for the formation of elaborate quasi-superlattice metal-oxide channels (i.e., various multilayer stacks composed of $\operatorname{In}_{2} \mathrm{O}_{3}, \mathrm{Ga}_{2} \mathrm{O}_{3}$, and $\mathrm{ZnO}){ }^{[67]}$ Optimized multilayer TFTs processed on both $\mathrm{SiO}_{2}$ and PEN substrates exhibited maximum electron mobility of 37 and $11 \mathrm{~cm}^{2} \mathrm{~V}^{-1} \mathrm{~s}^{-1}$, respectively. Dong et al. fabricated TFTs using $\mathrm{In}_{2} \mathrm{O}_{3}$ deposited on top of $\mathrm{ZrO}_{x}$ where both layers were processed via DUV in air for 80-90 min. Although no intentional TA was applied, the authors reported a sample temperature increase to about $50{ }^{\circ} \mathrm{C}$ during the treatment. Resulting devices showed good performance with electron mobility of $1.65 \mathrm{~cm}^{2} \mathrm{~V}^{-1} \mathrm{~s}^{-1}$ and channel current on/off ratio up to $10^{5} .{ }^{[37]}$

The DUV treatment without intentional heating was also investigated for various other metal-oxide semiconductors. ${ }^{[18,48,63,64,66]}$ As introduced by Kim et al. in 2012, ${ }^{[18]}$ various oxide films were fabricated by DUV irradiation in $\mathrm{N}_{2}$. Transistors incorporating an atomic layer deposited (ALD) $\mathrm{Al}_{2} \mathrm{O}_{3}$ dielectric and various photoconverted metal oxides as the channel materials showed high electron mobility values of $8.76,4.43$, and $11.29 \mathrm{~cm}^{2} \mathrm{~V}^{-1} \mathrm{~s}^{-1}$ for IGZO, IZO, and $\ln _{2} \mathrm{O}_{3}$, respectively. The unintentional heating of the substrates during the DUV treatment was measured and reached up to $150{ }^{\circ} \mathrm{C}$. This added thermal energy turned out to be an essential ingredient that leads to high performing TFTs. IGZO transistors and seven-stage ring oscillators were also prepared on polyarylate substrates, yielding electron mobility of $3.77 \mathrm{~cm}^{2} \mathrm{~V}^{-1} \mathrm{~s}^{-1}$, and propagation stage delay of $<210$ ns. Moreover, the same team used the DUV technique to produce IGZO as well as a zirconium aluminum oxide (ZAO) dielectric on thin polyimide substrates. ${ }^{[66]}$ TFTs, based on this combination, exhibited high mobility of $8.58 \mathrm{~cm}^{2} \mathrm{~V}^{-1} \mathrm{~s}^{-1}$ (Figure 3c). In a comparison between $2 \mathrm{ME}$ and water as solvents for DUV treated IGZO precursors, as well as between zinc nitrate and zinc acetate as the $\mathrm{Zn}$ source, it was found that due to a combination of broadened UV absorption and reduced carbon impurities after the photonic treatment, the combination of a water-based solution and nitrate precursor led to optimal TFT performance. ${ }^{[63]}$

In a series of experiments performed by John et al. ${ }^{[48]} \mathrm{In}_{2} \mathrm{O}_{3}$ and IZO transistors were produced via DUV irradiation of nitrate precursors in 2-ME. Despite the ambient air environment and the relatively low irradiance $\left.(16 \mathrm{~mW} \mathrm{~cm})^{-2}\right)$, functional TFTs were demonstrated only after $15 \mathrm{~s}$ of illumination, and high mobility of $>30 \mathrm{~cm}^{2} \mathrm{~V}^{-1} \mathrm{~s}^{-1}$ was reached after $15 \mathrm{~min}$ irradiation time. UV induced structural relaxation $\left(\mathrm{In}_{2} \mathrm{O}_{3}\right.$ films started to become crystalline) together with the formation of shallow donors and passivation of subgap states were proposed as the root cause for the good performance. Finally, Lee et al. ${ }^{[64]}$ demonstrated TFTs based on a bilayer channel architecture composed of a $\mathrm{GaO}_{x}$ layer deposited atop an $\mathrm{In}_{2} \mathrm{O}_{3}$ layer. Each layer was processed from nitrate precursors and subjected to 120 min DUV treatment in $\mathrm{N}_{2}$. This led to increased mobility performance of $11.2 \mathrm{~cm}^{2} \mathrm{~V}^{-1} \mathrm{~s}^{-1}$ for the $\mathrm{In}_{2} \mathrm{O}_{3} / \mathrm{GaO}_{x}$ bilayer 


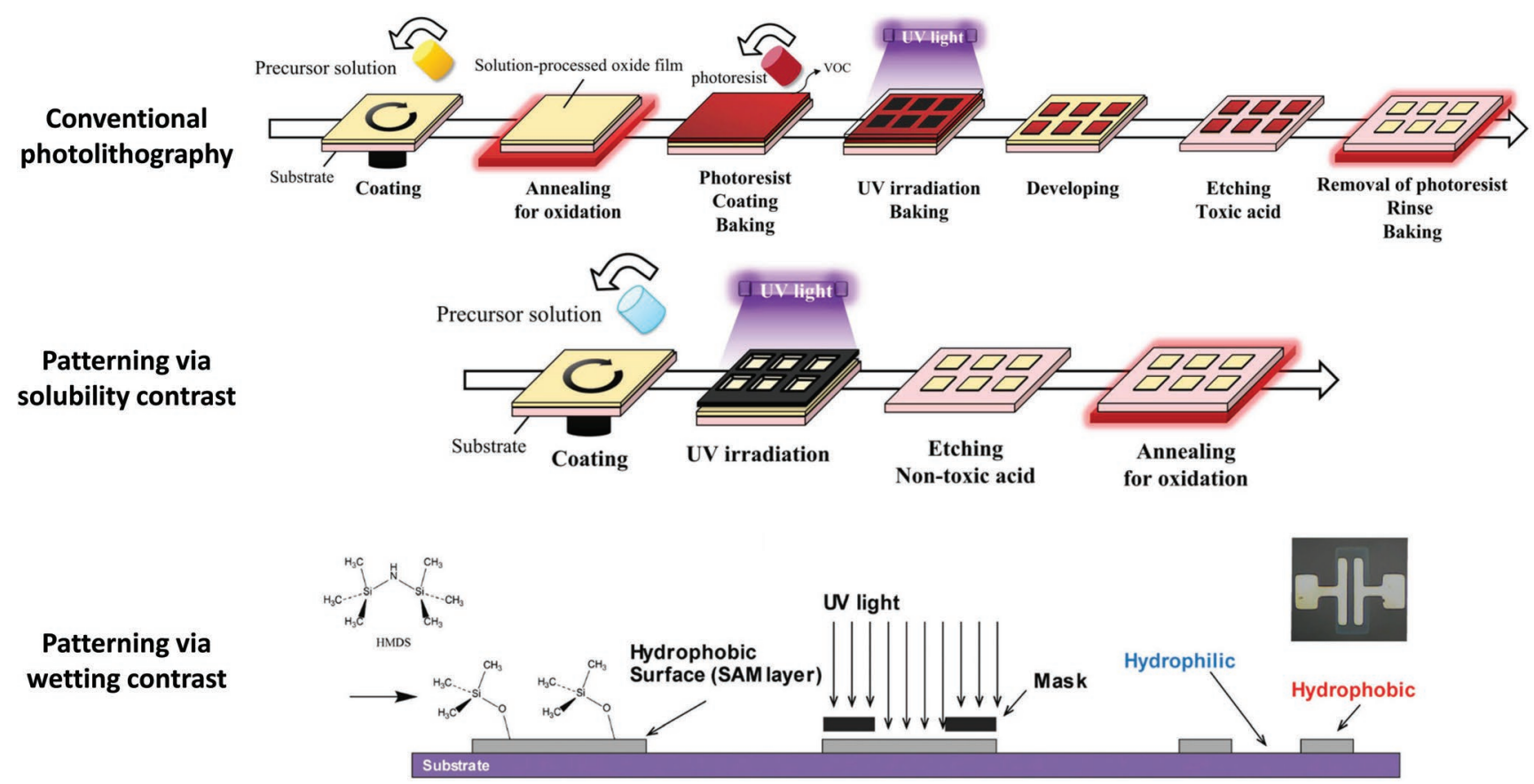

Figure 4. Schematic depiction of the processing steps used to pattern the metal-oxide films using either conventional photolithography (top), direct photonic patterning via solubility contrast (middle) (Adapted under the terms of the Creative Commons Attribution 4.0 International License. ${ }^{[70]}$ Copyright 2018, The Author(s). Published by Springer Nature) or via DUV induced wetting contrast (bottom). Reproduced with permission. ${ }^{\text {[1] }}$ Copyright 2017, Royal Society of Chemistry.

devices compared to $3 \mathrm{~cm}^{2} \mathrm{~V}^{-1} \mathrm{~s}^{-1}$ measured for the control device based on a single layer of $\operatorname{In}_{2} \mathrm{O}_{3}$ as the channel.

\subsubsection{Application of DUV as a Postdeposition Process Step}

The DUV technique may also be employed as a postdeposition step for vacuum deposited films, ${ }^{[3,77,78,80]}$ and solutionprocessed nanoparticles (NPs) ${ }^{[52,81]} \mathrm{Wu}$ et al. have shown that DUV treatment smoothens the surface of sputtered $\mathrm{ZnO}$ films and improves the adhesion of subsequently processed films which is critical for TFT fabrication. ${ }^{[78]}$ Furthermore, IGZO layers subjected to DUV irradiation exhibits permanent $n$-type doping characteristics due to the formation of surface defects, which under certain circumstances, can be exploited for improving the performance of the resulting TFTs. ${ }^{[80]}$ In another study, simultaneous ultraviolet and TA were applied to activate the sputtered IGZO films by facilitating the reorganization of metal-oxide bonds and reducing the density of defects. IGZO TFTs prepared by this method on polyimide substrates exhibit increased electron mobility and current on-off ratio as well as a positive $\Delta V_{\mathrm{TH}} \cdot{ }^{[39]} \mathrm{DUV}$ irradiation was also applied to $\mathrm{ZnO}$ NP-based TFTs as a mean for removing the insulating capping agents ${ }^{[52]}$ or combined with vacuum annealing to remove the unwanted and highly insulating organic ligands. ${ }^{[81]}$

\subsection{Use of DUV Light for Metal-Oxide Patterning}

The fabrication of electronic devices such as TFTs relies on the ability to deposit multiple types of materials (metals, dielectrics, semiconductors, etc.) sequentially with a high spatial resolution. Therefore, methods that combine facile and scalable patterning capabilities are potentially extremely valuable for the emerging field of large-area electronics. To date, the industry standard is conventional photolithography via lift-off or etching (Figure 4). Although widely used, conventional photolithography is complex and requires a clean environment where each step can be repeated reliably and consecutively. Therefore, any process steps that can be omitted could potentially simplify processing and hence the economics of manufacturing.

To date, two different approaches that rely on the use of DUV to pattern solution-processed metal-oxide films have been reported (Figure 4):

i) Direct light patterning through solubility contrast: ${ }^{27,40,42,43,53,54,61,65,70,72]}$ Here, exposure of the precursor layer to DUV irradiation is altering the solubility of the film in the exposed areas due to partial photoconversion of the precursor to an $\mathrm{M}-\mathrm{O}-\mathrm{M}$ network. Following a few minutes of exposure through a shadow mask, the unexposed areas of the precursor film remain soluble and can be dissolved/etched using common solvents or dilute etchant solutions.

ii) Patterning through surface wetting contrast: ${ }^{[46,71,76]}$ Substrate exposure to DUV increases the surface energy as compared to nonexposed areas. This process can be mediated via the use of different surfactants (e.g., self-assembling monolayers) and substrate materials. Consequently, the solution processed precursor only wets the pre-exposed areas without the need for further etching processes.

Both approaches are comparatively less demanding than standard photolithography as they offer fewer process steps 
and more environmentally friendly etching protocols while alleviating possible contaminations from residual photoresist. In either case, following the patterning process, a thermal annealing step is carried out to convert the precursor to the targeted stoichiometric metal oxide.

In the following, we are discussing various aspects of direct photopatterning via solubility contrast. First, we follow the progress from the patterning of single films to fully photopatterned TFTs, including the source/drain, gate and dielectric materials. Then we are discussing photoactive precursors that do not rely on the common photosensitizer BzAc and AcAc. This is followed by the review of specific process conditions.

The suitability of using DUV induced solubility contrast was initially explored in 1994 by Tohge et al. ${ }^{[56]}$ and Shinmou et al. ${ }^{[57]}$ who used alkoxide precursors photosensitized with benzoylacetone or acetylacetonate to create fine patterns of $\mathrm{ZrO}_{x}$ and $\mathrm{TiO}_{2}$ with a resolution up to approximately $10 \mu \mathrm{m}$. The patterning of other metal oxides was soon after explored and expanded to transparent conductive oxides, such as ITO ${ }^{[59]}$ or antimony doped $\mathrm{SnO}_{2} \cdot{ }^{[58]}$ Fully functional TFTs incorporating directly photopatterned IGZO and $\mathrm{In}_{2} \mathrm{O}_{3}$ channel materials were demonstrated by Rim et al. in 2013 (Figure 5a,b). ${ }^{[40]}$ To achieve this, they used a combination of a photosensitizer and combustion synthesis by adding AcAc and nitric acid into the precursor solution. Flexible $\mathrm{In}_{2} \mathrm{O}_{3}$ TFTs on polyimide were also successfully demonstrated with electron mobility of $2.24 \mathrm{~cm}^{2} \mathrm{~V}^{-1} \mathrm{~s}^{-1}$. The same group also combined photopatterned conductive ITO as source/drain electrodes with photopatterned ZTO as the semiconductor, at a resolution of $5 \mu \mathrm{m}$ (Figure $5 \mathrm{c}, \mathrm{d}$ ). ${ }^{[43]}$
Going one step further, the same team combined IGZO as the channel material with $\mathrm{AlO}_{x}$ and ITO as the dielectric and conductive electrodes, to demonstrate photopatterned all-oxide TFTs on flexible polyimide substrates..$^{[27]}$

Although BzAc and AcAc are the most widely used additives for photopatterning of metal oxides, recent research has extended to other materials. For example, Jeong et al. used zinc diacrylate as a photo cross-linkable precursor. ${ }^{[53]}$ Following DUV exposure and thermal annealing at $450{ }^{\circ} \mathrm{C}$, IZO layers were deposited onto $\mathrm{SiO}_{2}$ dielectric to produce TFTs with electron mobility of $0.8 \mathrm{~cm}^{2} \mathrm{~V}^{-1} \mathrm{~s}^{-1}$. Changing the gate dielectric to an ion-gel system yielded TFTs with maximum mobility of $7.1 \mathrm{~cm}^{2} \mathrm{~V}^{-1} \mathrm{~s}^{-1}$. Using novel methoxyiminopropionat ligands (metal oximates) of indium, zinc and tin, Sanctis et al. realized photopatterned IZO and ZTO transistors at $350{ }^{\circ} \mathrm{C}$, with $\mu=7.8$ and $3.6 \mathrm{~cm}^{2} \mathrm{~V}^{-1} \mathrm{~s}^{-1}$, respectively, and current on/off ratios higher than $10^{7}{ }^{\left[{ }^{-12]}\right.}$ In a separate study Kim et al., used DUV to pattern IZO films using nitrate-based precursors with added AcAc as a photosensitizer and compared it to that of pristine indium acetylacetonate and zinc acetylacetonate hydrate precursors. ${ }^{[65]}$ They found that TFTs made from pristine precursors showed improved electron mobility as compared to IZO TFTs made with the use of photosensitizer (0.32 vs $0.03 \mathrm{~cm}^{2} \mathrm{~V}^{-1} \mathrm{~s}^{-1}$ ) accompanied by improved current on/off ratios and subthreshold swing. The performance improvements were attributed to the reduced concentration of residual impurities within the oxide layers due to the absence of the photosensitizer. Similar, carbon-free DUV patterned IGZO transistors were produced by Miyakawa et al. through the use
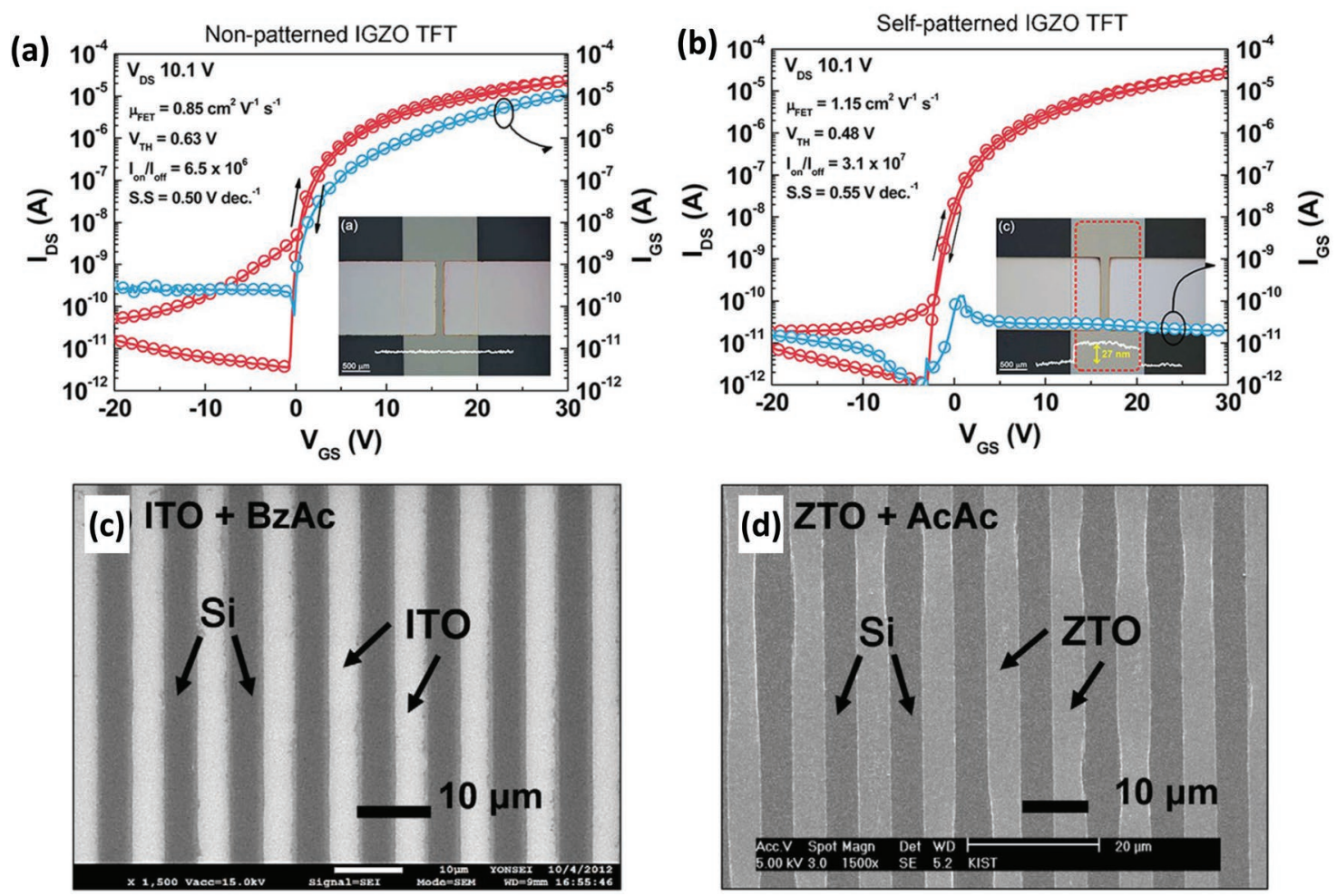

Figure 5. Transfer characteristics of ICZO TFTs with: a) unpatterned and b) photochemically patterned ICZO layers. Patterned TFTs exhibit significantly reduced gate current leakage. Reproduced with permission. ${ }^{[40]}$ Copyright 2013, American Chemical Society. The patterns of c) ITO and d) ZTO are both fabricated using direct photopatterning via solubility contrast. Reproduced with permission. ${ }^{[43]}$ Copyright 2015, Springer Nature. 
of water as the solvent in combination with a nitrate-based precursor. $^{[70]}$ Following layer deposition via spin-coating, the solid precursor films were only dried at $60^{\circ} \mathrm{C}$ prior to the DUV irradiation. Generation of free radicals of water moieties as well as nitrate ligands during DUV irradiation was thought to initiate $\mathrm{M}-\mathrm{O}-\mathrm{M}$ network formation. IGZO TFTs on flexible PI films were demonstrated with electron mobility of $4.8 \mathrm{~cm}^{2} \mathrm{~V}^{-1} \mathrm{~s}^{-1}$.

When processing $\mathrm{AlO}_{x}$ and $\mathrm{In}_{2} \mathrm{O}_{3}$ from nitrate precursors, Daunis et al. found the DUV irradiation time required to achieve sufficient solubility contrast for patterning could be reduced from 20 to $10 \mathrm{~min}$ when combustion additives, such as AcAc and ammonium, were added. ${ }^{[61]}$ They showed $\mathrm{In}_{2} \mathrm{O}_{3}$ TFTs fabricated on a flexible shape memory polymer substrate from which a high electron mobility value of $86 \mathrm{~cm}^{2} \mathrm{~V}^{-1} \mathrm{~s}^{-1}$ was obtained, although the geometric capacitance of the $\mathrm{AlO}_{x}$ gate dielectric exhibited high dispersity in the lower frequency range which could potentially result to erroneous mobility calculations.

In the case of more complex material systems such as ternary and quaternary metal oxides, it is important to be able to control the elemental composition of both the precursor and the post-DUV patterned material. To this end, Wang et al. reported zinc loss in their formed IGZO films following the removal of the unexposed areas in ethanol (i.e., during the development step). ${ }^{[42]}$ When the irradiation time was very short, the $\mathrm{Zn} /$ In ratio was reduced from 0.5 to 0.05 . Since the sources for In and $\mathrm{Ga}$ were nitrates and the source for $\mathrm{Zn}$ was zinc acetate, it was argued that the comparably weaker network formation of acetates, as compared to nitrates, was proposed as the reason behind the stoichiometric change. By increasing the DUV irradiation time from 30 to $40 \mathrm{~s}$, the Zn loss behavior could be successfully suppressed. This demonstrated the versatility of DUV method for also tuning the chemical composition of complex metal-oxide systems. In the case of UV induced wetting contrast, such compositional changes are of no concern. This is because, in the latter method, the substrate is typically first treated with a surface modifier (e.g., a self-assembling monolayer (SAM)), the sole purpose of which is to reduce its surface energy, i.e., making it more hydrophobic. DUV irradiation through a mask is then applied to remove the SAM leaving behind the predefined patterns of hydrophilic/hydrophobic areas. Consequently, the precursor solution processed on top of the substrate only wets the hydrophilic areas, whereas the remaining area stays free of precursor formulation. The process is schematically depicted in Figure 4. Drying effects and edge bead formation during solution deposition was shown to lead to nonuniformities. ${ }^{[82]}$

In 2009, Park et al. used UV induced wetting contrast in combination with octadecyltrichlorosilane SAM to pattern Ag nanoparticulate-based source and drain electrodes and subsequently the IGZO semiconductor via a dip coating process. ${ }^{[76]}$ TFTs with channel lengths of down to $10 \mu \mathrm{m}$ were demonstrated and electron mobility and current on/off ratios of $0.2 \mathrm{~cm}^{2} \mathrm{~V}^{-1} \mathrm{~s}^{-1}$ and $>10^{6}$, respectively, were achieved. In a similar approach, Park and Noh showed 4-inch wafer-scale processing of IGZO by combining DUV patterned hexamethyldisilazane SAM with wire bar coating. ${ }^{[71]}$ Optimized bar-coating parameters rendered high mobility TFTs (up to $\mu=12.78 \mathrm{~cm}^{2} \mathrm{~V}^{-1} \mathrm{~s}^{-1}$ ) with good uniformity across the wafer. More recently, Lee et al. showed the possibility of a direct patterning approach without SAM modification of the surface. ${ }^{[46]}$ The wetting contrast was achieved by directly irradiating the surface of $\mathrm{Si} / \mathrm{SiO}_{2}$ wafers through a mask, causing a difference in surface energy between exposed/ unexposed areas of 7-8 $\mathrm{mN} \mathrm{m}^{-1}$. This difference was sufficient to allow selective wetting in exposed areas and to pattern/convert IGZO films. A drawback of this method is that a significant widening of patterned features relative to the mask design was found. Nevertheless, TFTs with $\mu=1.4 \mathrm{~cm}^{2} \mathrm{~V}^{-1} \mathrm{~s}^{-1}$ and channel current on/off ratios of $10^{8}$, were demonstrated. Importantly, patterning of the semiconductor mostly results in reduced gate leakage currents, which is important for application in largearea electronics. To this end, and regardless of the conversion method used, nonpatterned semiconductor-based TFT always feature significantly larger gate leakage currents (Figure 5a) than devices utilizing patterned oxide channels (Figure 5b). An added advantage of patterned metal-oxide TFTs is that the fringe/parasitic currents (a form of leakage current) are significantly suppressed hence preventing erroneous mobility calculations.

\section{Laser Annealing}

\subsection{A Brief Introduction to Laser Annealing}

Stemming from the existing, well established, research regarding its application on conventional electronic materials, ${ }^{[83-93]}$ the implementation of LA in metal oxides ${ }^{[2,94-97]}$ has been continuously accompanying the evolution of novel materials and fabrication techniques over the past few decades. The remarkable characteristics of this promising annealing method match the high thermal energy demand of metal-oxide thinfilm manufacturing, alongside other advantageous characteristics such as decreased processing time, compatibility with R2R and S2S printing techniques, low processing temperatures, precise energy delivery control, materials' selectivity, and direct patterning. ${ }^{\text {98-102] }}$ Therefore, the introduction of LA in metaloxide-based applications has offered improved optoelectronic characteristics, thus inspiring the next generation of (opto) electronic devices including TFTs, organic light emitting diodes OLEDs, sensors, capacitors, electrochromics, memory devices, and photovoltaics. ${ }^{[103-110]}$

Whether considering metal-oxide semiconductors, dielectrics and conductors, the essential role of LA onto their transition into high-quality films has been extensively investigated. ${ }^{[11-117]}$ Particularly in solution processes (sol-gels, etc.), the parallel evolution of manufacturing techniques has triggered a new era of large-scale fabrication of metal-oxide film-based devices, as well as a demand for time and cost-efficient approaches of inducing the required photochemical transition of the precursors into high-quality metal oxides. Therefore, LA, by fulfilling the aforementioned requirements, as well as being compatible with flexible substrate utilization, has become one of the most promising processing paradigms for metal-oxide electronics. Nevertheless, the route into achieving the optimized film characteristics demands special attention to the underlying physical phenomena in order to design the most effective LA process. Thus, multiple studies have been focusing on the correlation between the experimental parameters and the exact mechanism of metaloxide film conversion/formation, successfully exhibiting optimal characteristics, as well as enhanced device performance. ${ }^{[113,118]}$ 


\subsection{The Laser Annealing System}

A research grade laser annealing system can be a complex and expensive experimental apparatus involving several elements and a variety of beam path configurations, that allows investigating the multiple experimental parameters involved in the process, in order to identify the most efficient LA recipe for a given material or device. Once a robust recipe is identified a custom-designed system that can deliver a specific LA process in a potential mass volume production line can be manufactured, for a fraction of the cost in comparison to the research tool. A generic LA experimental set-up could be divided into three parts: a) the laser source, b) the beam delivery system, and c) the sample manipulator. The first part would include the source of the laser light and depending on the type of laser it will offer variability of some of the experimental parameters in question (e.g., repetition rate, pulse intensity, wavelength, etc.). The second part, the beam delivery system, is often the most complex part. It serves three purposes: i) it receives the raw beam from the laser and manipulates it in order to give the characteristics needed for the processing, ii) it delivers the suitably manipulated beam to the sample, and iii) it comprises any online diagnostics for the monitoring of the critical characteristics of the process. Finally, the third part, the sample manipulator, houses the sample appropriately and manipulates it as needed for the process. Translation stages (or rollers in the case of R2R systems) are often involved and contribute to the sample environment (temperature, etc.) in order to achieve the desired annealing result.

An example of such a system, which takes into account most of the aforementioned parameters, is shown in Figure 6. In this system, the laser is a $\mathrm{KrF}$ Excimer Laser (248 nm) and the beam delivery system comprises a variable attenuator (employs a partially reflective first plate and a second plate that compensates for the parallel displacement of the beam caused by the first plate), a beam homogenizer (employs two lens arrays and a condenser lens which provides a top-hat profile on both axes), a stage for interchangeable masks that provide various spot shapes onto the sample and a combination of field and projection lenses that will define the size of the mask shape onto the sample. The sample sits inside a pressure cell on an $X-Y-Z$ translational stage which allows for the introduction of various gases during LA. Alternatively, $X-Y$ motion can be achieved by moving the beam with the use of flying or rotating optics (aka "galvo") while the stage remains stationary.

Note that here we do not consider an additional set of parameters relating to the materials' optical and thermal properties, geometrical configurations (e.g., design of optical spacers and mirror designs to enhance absorption) or the type and characteristics of its substrate. These parameters related to the LA system can be divided into three categories (reflecting the three parts of a generic laser annealing system): i) laser unit parameters (wavelength, polarization, pulse duration and repetition rate), ii) laser beam parameters (fluence, number of pulses, spot size and shape), and iii) environmental parameters (atmosphere, pressure and temperature) (Figure 7). A more detailed description of each of the parameters shown in Figure 7 follows.

- Laser Wavelength: In most cases, the wavelength of the laser light (nm) is in the UV region of the spectrum. This ensures the highest possible energy absorption by most metal oxides and their precursors via different physical processes. If a given material presents intraband absorption, then this could also be exploited. Therefore, a good starting point is the evaluation of the absorption spectrum of the precursor/material used.

- Light Polarization: The polarization of the light utilized will have an effect on the penetration depth in the sample and hence is expected to have an effect on the heat generation profile. Assuming that the material is either amorphous or polycrystalline, linearly polarized light is expected to penetrate deeper in the sample/layer, whereas naturally polarized light (unpolarized) will be absorbed within a thinner part of the sample. Circularly polarized light is equally expected to be absorbed closer to the surface, compared to linearly polarized light.

- Pulse Duration: This is the duration of the optical pulse (also referred to as pulse length) and in essence is the period of time during which laser light interacts with the material. The pulsed laser annealing systems lie in the nanosecond (ns) regime (2-30 ns), which is known to induce thermal phenomena, in contrast to shorter pulse duration (ps and fs) lasers, which are known to induce cold ablation, through the breaking of chemical bonds. Nevertheless, in some extreme situations, ultrashort pulses created by compression of longer pulses, may also be suitable in inducing multi-photon absorption, with desired thermal effects rather than cold ablation.

- Repetition Rate: The repetition rate is the pulse frequency in $\mathrm{Hz}$ that the laser operates at, which is usually dictated by the laser pumping. Typically used in laser annealing are repetition rates from a few $\mathrm{Hz}$ to a few hundred $\mathrm{Hz}$. However, as of recent years, more laser sources offering ultrahigh repetition rates are becoming available, reaching repetition rates of a few hundreds of $\mathrm{kHz}$ [also commercially known as

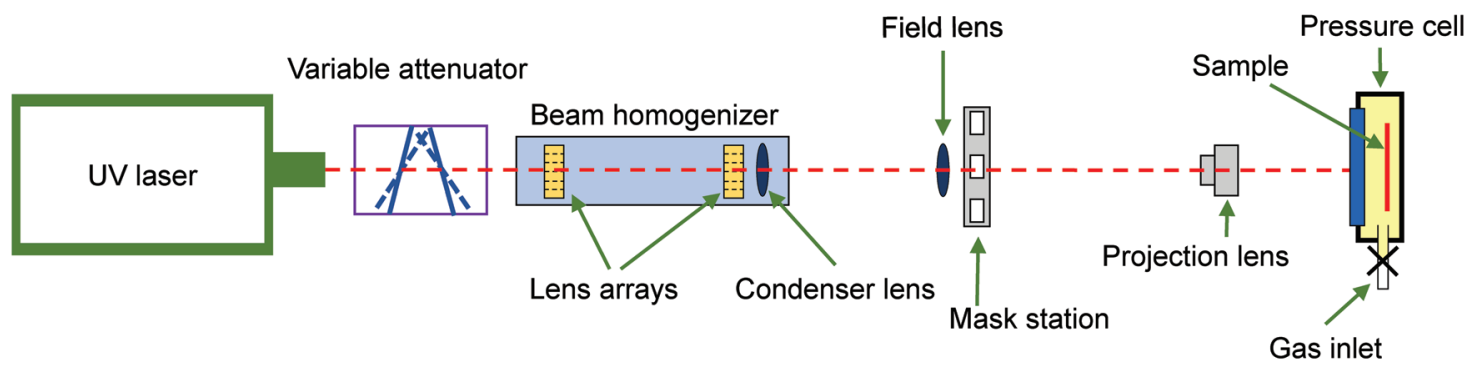

Figure 6. A typical laser annealing experimental setup. 


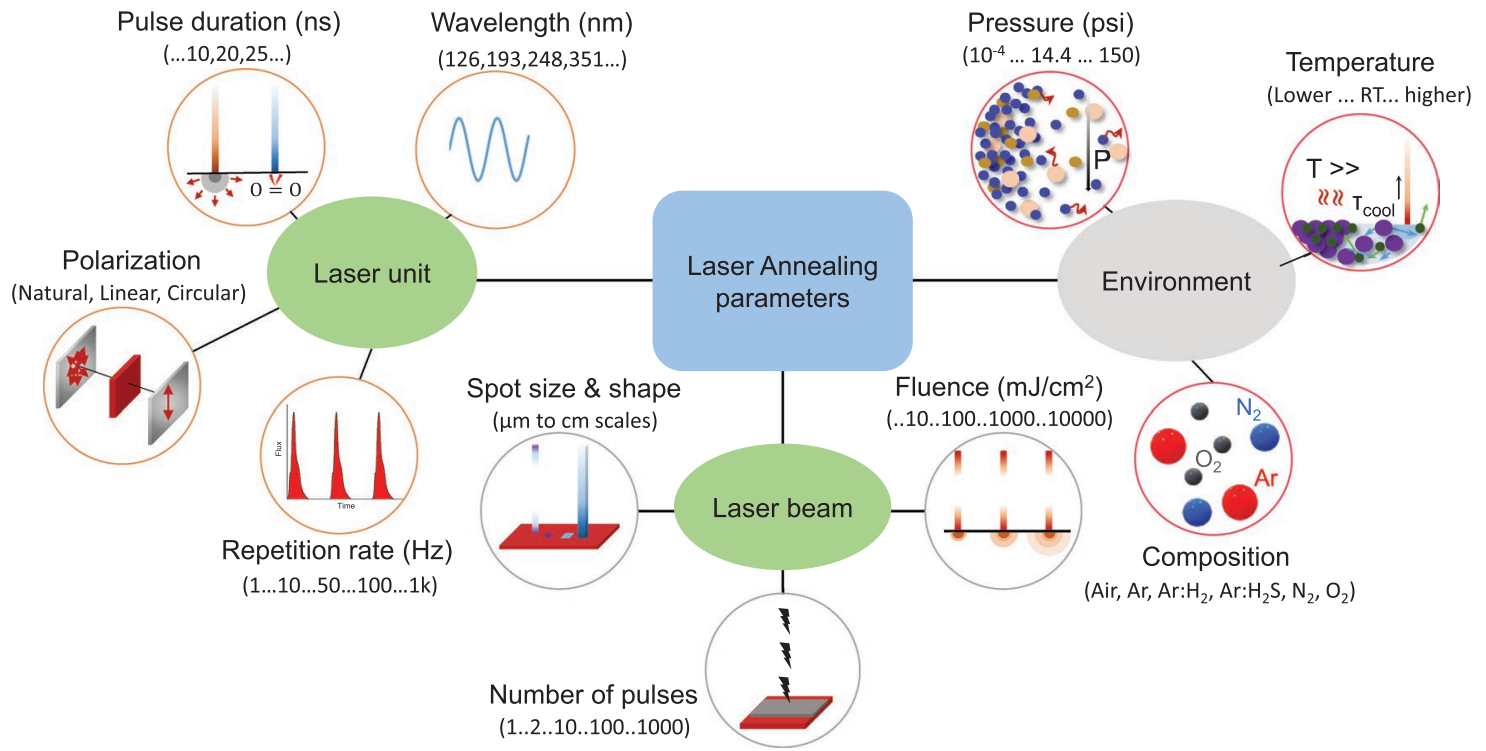

Figure 7. Important parameters associated with the laser annealing process.

quasicontinuous wave $(\mathrm{CW})]$. The repetition rate is clearly of critical importance in processes where more than one pulse is required, as allowing or not permitting a complete thermal relaxation of the sample prior to the next pulse, will have a different effect on the heating of the sample.

- Laser Fluence: Pulsed lasers are characterized by the amount of energy emitted per pulse (in $\mathrm{mJ}$ per pulse). The energy packed in each laser pulse can be utilized to irradiate different size areas (as per the laser spot size on the sample). Hence, for an accurate and transferable description of the incident energy on the sample the energy density (i.e., the

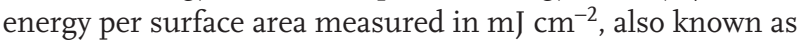
fluence) must be reported.

- Number of Pulses: A certain area of the sample could be irradiated more than once. The total applied number of pulses delivered to the same area of the sample defines the total energy packet transferred. The effect of each subsequent pulse is not expected to be the same, as in an effective annealing process the sample is modified by each of the previous pulses. Changes are expected both to the sample's optical properties (becomes more or less absorbing) and/or its thermal properties (changes to its thermal capacity and thermal conductivity). Additionally, in a multi pulse process, it is not necessary to utilize pulses of constant fluence, since the sample may benefit by pulses of higher or lower fluence, as its properties are evolving from subsequent pulses.

- Laser Spot: The characteristics of the laser spot upon delivery to the sample, like size shape and energy distribution within it, are expected to have different effects on the sample. The area of the laser spot may be filled with a Gaussian-like energy density profile or be of highly uniform energy density (top-hat profile). In some cases, the laser spot may include hot spots, i.e., smaller areas within its footprint of higher energy density, but this is largely undesirable hence some homogenization technique would be enlisted. The laser spot size and shape will determine the manner in which a large area of the sample will be filled, with raster scanning or line scanning being possible. If the sample contains selected areas (features) larger than the laser spot size requiring annealing, then a step and repeat process is applied. Special attention must be given to any edge effect that can be common with laser annealing. In this case, we refer to heating effects to the area immediately next to the laser spot, but outside its footprint. Particularly in the case of utilizing laser microspots for annealing, the overall result may be that of the edge effect rather than of the laser spot itself.

- Temperature: This is the temperature at which the sample is kept during LA. Room temperature offers the least complications (experimentally), but elevated or reduced temperatures may offer benefits by slowing down or speeding up, respectively, the cooling of the sample immediately after the annealing has occurred. Crucially one of the defining factors of any annealing process is the cooling rate, with the maximum temperature reached and the time it is sustained (dwell time) being also of importance; the role of the substrate is essential for the cooling rate. ${ }^{[28]}$

- Pressure: The pressure at which the sample is kept during LA. In certain cases, high-pressure application during LA (of any gaseous environment) may be advantageous to avoid any detrimental effects of ablation. A highly pressurized environment will raise the ablation threshold to higher fluences, permitting highly energetic annealing that would otherwise damage the sample. It is also possible, depending on the process, for the pressure to define the final characteristics of the material being processed.

- Environment Composition: It is essential, sometimes, to perform LA under an inert atmosphere (such as Ar or $\mathrm{N}_{2}$ ) or in other cases in an oxidizing (up to $100 \% \mathrm{O}_{2}$ ), a reducing (few $\% \mathrm{H}_{2}$ in an inert balance) or a highly reactive environment (up to $100 \% \mathrm{NH}_{3}$ ) in order to either protect the sample from ambient atmospheric gases or to induce specific chemical reactions. Control and alteration of the sample's stoichiometry is possible by selecting the environment composition accordingly. 


\subsection{Laser-Matter Interactions}

One of the key issues in LA is the spatial distribution of the energy delivered from the laser pulse and the precise temporal temperature profile generated in the targeted material/structure due to this energy. Knowing how the temperature profiles are affected and interact with the sample's constituents and/or geometrical features (e.g., single layer or multilayer and their thickness), one can extract valuable insights about the underlying mechanisms of LA. Those mechanisms influence the oxide crystal structure and structural defects, upon LA, i.e., LA probes mostly physical properties. In the case of sol-gel metaloxide thin films, the effect of LA principally involves the photochemical conversion of as-spun metal-oxide precursor films into the metal-oxide phase, i.e., LA acting mostly as a chemical process, followed by its crystallization and densification via physical processes similar to vacuum deposited MO films. This additional step (photochemical conversion) highlights the further complexity of sol-gel metal-oxide film growth, accompanied by the importance of the LA effect. In the systems of interest, several effects such as material optical absorption and interference contribute to the energy absorption distribution, while the materials' thermal properties, such as specific heat capacity and thermal conductivity and the laser pulse profile, determine the temperature transients developed. ${ }^{[119-121]}$

In this section, we discuss qualitatively the underlying physical phenomena that determine these photothermal processes involved in LA. As in any annealing process, the temperature profile generated is of utmost importance. This temperature profile depends primarily on the materials optical (complex refractive index) and thermal (thermal conductivity and heat capacity) properties, as well as the geometrical features of the annealed structures (i.e., interference). These sample parameters, in addition to individual parameters included in each photonic processing technique (e.g., pulse duration in the case of LA and flash lamp, etc.) determine the temperature transients developed and the effect of the process to the metal-oxide film. As mentioned previously, the amount of heat induced during laser annealing relies predominantly on the absorption spectrum of the targeted material and hence on the laser wavelength used. Thus, a variety of absorption mechanisms can be utilized by tuning the laser's wavelength, in order to match the absorption spectral profile of the metal oxide. As an example, the complex refractive index $(\tilde{n}=n+i k)$ of $\mathrm{Sn}_{\mathrm{In}_{2} \mathrm{O}_{3}}$ (ITO) is plotted in Figure 8a.

The imaginary part of the complex refractive index (extinction coefficient), $k$, reveals the spectral space of transparency (where $k \approx 0$, in this case across the visible wavelengths, $400-700 \mathrm{~nm}$ ) and the spectral range of absorption (where $k>0$ ). Evidently, the available light absorption mechanisms can be intuitively summarized in the following cases: i) stronger interband absorption, enabled at UV wavelengths, and ii) weaker intraband (free-electron) absorption located, naturally for transparent conducting oxides, at longer wavelengths. These can be considered the intrinsic capabilities of the material itself (bulk properties). Practically, however, for the applications of interest, one should consider the case of a metal-oxide thin film on top of a substrate. For this case, we present the overall absorption
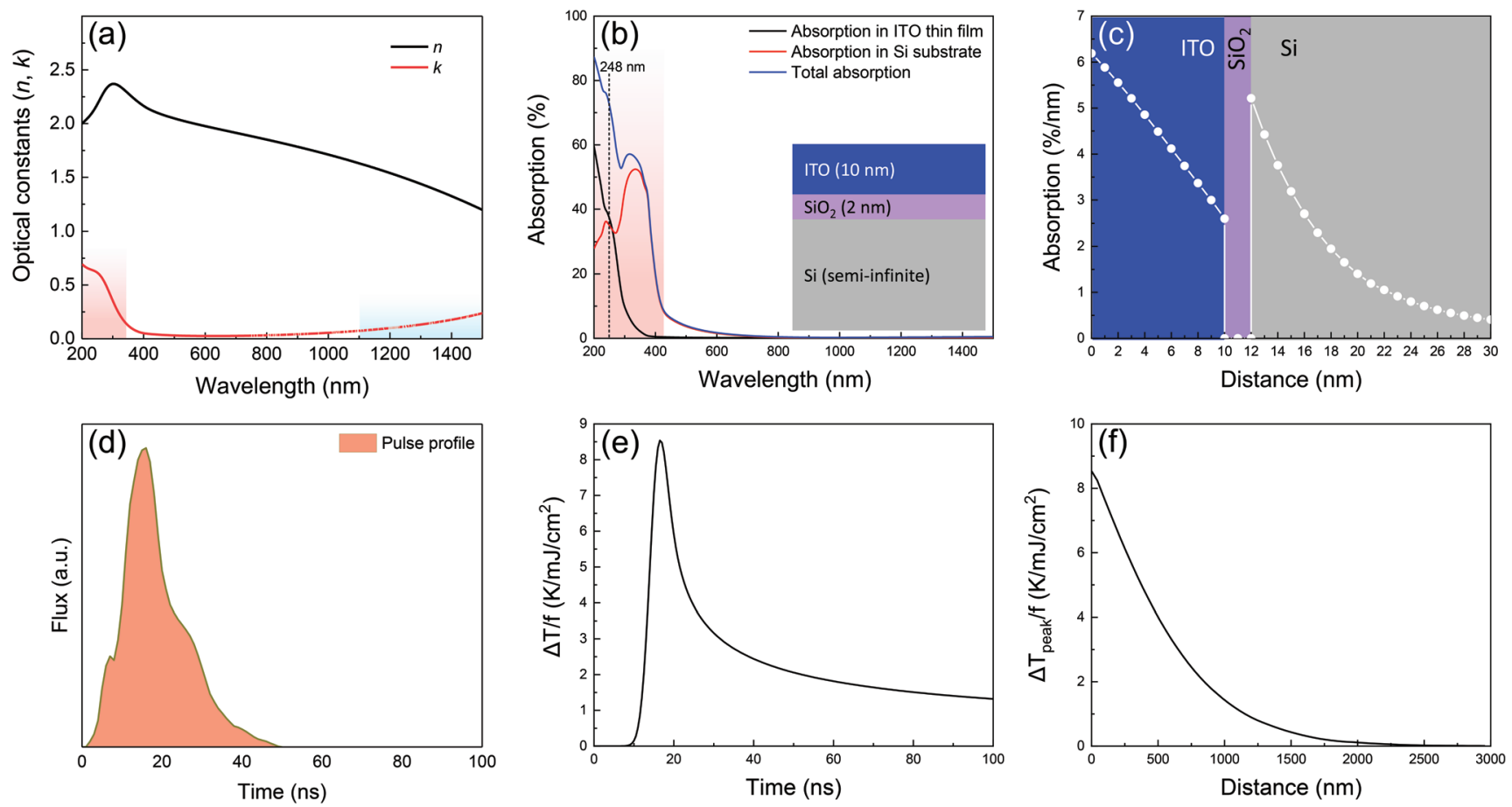

Figure 8. a) Optical constants $(n, k)$ of ITO derived by in-house ellipsometry measurements and appropriate modeling. b) Analytically calculated total absorption of a $10 \mathrm{~nm}$ thick ITO film on top of a Si wafer with a native oxide on top (blue solid line). The black and red solid lines demonstrate the explicit absorption in the ITO thin film and Si substrate, respectively. c) The spatial absorption distribution at $248 \mathrm{~nm}$ (typical for a KrF excimer laser) in a $10 \mathrm{~nm}$ thick ITO film on top of a Si wafer with a native oxide on top. d) The experimental temporal pulse profile of an excimer UV laser. e) Explicit normalized temperature transient at the top of the ITO film. f) Peak normalized temperature gradient in the ITO/Si structure. 
spectrum (total absorption) of an ITO thin film $(10 \mathrm{~nm})$ on top of a Si wafer with a $2 \mathrm{~nm}$ native oxide (blue solid line in Figure $8 \mathrm{~b}$ ). We also present the explicit absorption on the ITO thin film (black solid line in Figure 8b) and on the Si substrate (red solid line in Figure 8b). At such small thickness, the intraband absorption becomes negligible and the UV absorption becomes the dominant possible mechanism of absorption (to be noted that absorption in mid infrared wavelengths cannot be ignored as a means of heat transfer due to the phononpolariton modes which are manifested in that spectral regime). Note that $\mathrm{Si}$ absorbs below $400 \mathrm{~nm}$ contributing significantly to the total absorption and consequently to the heating of the system. Naturally, UV lasers (e.g., excimer lasers) are employed in metal-oxide processing, ${ }^{[122-127]}$ no matter of their electronic properties (conductors, semiconductors, dielectrics). Additionally, and for the purpose of this review, we are interested in the thermal effects induced upon LA, thus we are focusing our attention to the ns pulse regime; nevertheless. Nevertheless, the utilization of ${ }^{p s}{ }^{[128]} \mathrm{fs},{ }^{[129-131]}$ and $\mathrm{CW}^{[100,132-145]}$ lasers for metal-oxide film processing is additionally reported. It is, therefore, worth addressing the connection between $\mathrm{CW}$ and pulsed LA, as a scanning CW process shares similar principles with pulsed LA albeit with significantly lower peak power, defined by the scanning speed and laser spot size rendering a ms interaction with the film (in contrast to the typical ns pulses provided by excimer lasers).

A further point of discussion is the maximum temperature rise within the structure, which eventually determines the effectiveness of the LA process. Fundamentally, one needs to integrate the light propagation properties (Figure 8a), in order to define the detailed spatial absorption distribution within the structure (Figure 8c), with the experimental laser temporal profile (Figure $8 \mathrm{~d}$ ) and solve the 1D heat transport equation, in order to arrive to the temperature transients at each point in the structure. In more detail, the obtained absorption spatial distribution, $a(z)$, (Figure 8c) combined with the experimental laser pulse profile $\varphi(t)$ are used as the laser induced heating source $Q(z, t)=a(z) \varphi(t)$ and can be solved numerically in the 1D heat diffusion equation

$c(z) \rho(z) \frac{\partial T(z, t)}{\partial t}=\frac{\partial}{\partial z}\left[k(z) T(z, t) \frac{\partial T(z, t)}{\partial z}\right]+Q(z, t)$

where $T$ is the local temperature transient, $\rho$ is the mass density, $c$ is the heat capacity, $k$ is the thermal conductivity and they are in general both space (due to the interchange between different materials) and temperature dependent. $\partial t, \partial z$ denote partial derivatives with respect to $t$ and $z$, respectively (where $t$, $z$ are the time and distance from the top surface, respectively). The total laser fluence is given by $f=\int_{-\infty}^{+\infty} \varphi(t) \mathrm{d} t$.

Assuming a flat pulse profile, which is valid for relatively small spots and top hat beam profiles, the time integration of Equation (1) results at the transient temperature at each point in the multilayer volume. For simplicity, here we initially assume a linear (i.e., temperature independent) response, and normalize temperatures to the incident laser fluence $f$, i.e., we choose to present the normalized temperature transient per fluence in $\mathrm{K} \mathrm{cm}^{2} \mathrm{~mJ}^{-1}$ (Figure 8e) as it allows for more general and qualitative discussion serving the purpose of this review.
Studying the linear heat transport regime one can get insights into the temperature transients and gradients that can be developed, without considering nonlinear effects on the materials' properties, nor the thermodynamics of phase changes, e.g., melting and re-solidification. Such a temperature gradient is presented in Figure 8f. By comparing Figure 8c,e, we observe that although optically the radiation is absorbed within the ITO layer and the first few nm of the Si substrate (more than $99.99 \%$ of the $248 \mathrm{~nm}$ is absorbed) the thermal effect extends up to $2 \mu \mathrm{m}$ from the top free surface of the thin film and into the $\mathrm{Si}$ substrate. One can clearly appreciate the importance of this "heat wave" in the annealing process and its practical implications to metal-oxide processing.

Finally, regarding radiation and convection losses from the top surface, they are of no consequence and in-depth discussions and analyses of such phenomena have been reported elsewhere. ${ }^{[28,119,146,147]}$ A final note is that the 1D heat diffusion suffices to describe the temperature gradient in the case of thin films and substrates with high thermal conductivity. However, when lateral temperature distribution can no longer be ignored, the use of more sophisticated 2D or 3D models becomes more appropriate.

So far, we have discussed the implications of LA in solid media. However, application of the technique to sol-gel precursor films imposes the additional parameter of the photochemical conversion of the reagents into the stable metal-oxide phase. In brief, in a sol-gel process a metal-organic precursor solution is deposited onto the substrate, forming an intermediate phase, which subsequently requires enough energy to transform into metal-oxide films via a photothermally induced chemical reaction (comprising of organic residuals removal and subsequent generation of $\mathrm{M}-\mathrm{O}-\mathrm{M}$ bonds).

Following LA of the metal-oxide sol-gel precursor with ns laser pulses, the subsequent energy absorption leads to a local temperature increase, which initiates the chemical transition to the desirable metal-oxide phase. ${ }^{[99]}$ This photothermally induced chemical reaction differs from the photochemical phenomena occurring during fs LA, in which a rapid material ablation via instant bond breaking is induced, with limited temperature rise. ${ }^{[148-150]}$ As the literature refers to such phenomena as photochemical phenomena, attention must be given to distinguishing those two photochemical events (ablation and precursor conversion), which occur also at different time scales.

\subsection{Laser Annealing of Metal-Oxide Layers}

In this section, we discuss the application of LA for controlling the properties of various metal-oxide materials, including conductors, semiconductors, and dielectrics.

\subsubsection{Metal-Oxide Dielectrics}

The importance of dielectric and ferroelectric materials in novel, metal-oxide-based electrical devices has led to the investigation of their optoelectronic response, under ns laser treatment. ${ }^{[151-161]}$ Kang et al. studied the effect of KrF ns laser treatment on $\mathrm{Ba}_{0.6} \mathrm{Sr}_{0.4} \mathrm{Ti}_{0.3}$ (BST), a well-known ferroelectric 
material, especially for its use in thin-film capacitors. In brief, after the formation of sol-gel BST films (and their subsequent pyrolysis at $300{ }^{\circ} \mathrm{C}$ for $5 \mathrm{~min}$ ), various laser fluences up to

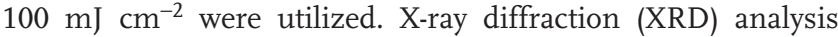
showed a remarkable crystallinity enhancement of the as-deposited amorphous BST films, especially after laser irradiation of $100 \mathrm{~mJ} \mathrm{~cm}{ }^{-2}$. The formation of grain size crystallites was subsequently confirmed, indicating the presence of 3 co-existing phases; an amorphous phase in the bottom of the BST film, which remained unaffected by the laser irradiation, and two crystallized regions on top of the film. The division of laser annealed BST films into different crystallinity regions widely influenced their dielectric permittivity values. Specifically, the presence of large grains on the top region increased the out-ofplane dielectric permittivity, which, was reportedly lower than expected due to the amorphous region. In order to exclude the amorphous phase influence on permittivity values, a measurement of in-plane permittivity was conducted. According to this measurement, in-plane dielectric permittivity values were sig-

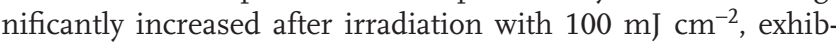
iting comparable values to epitaxial BST films grown by high temperature annealing processes. ${ }^{[160]}$

Amongst the studies aiming to deconvolute the crystallization mechanism of $\mathrm{SiO}_{2}$ after laser treatment via utilizing experimental data, Gavrylyuk et al. employed an additional theoretical approach regarding the effect of nanocomposite $\mathrm{SiO}_{2}$ formation on the surface of $\mathrm{SiO}_{x}$ after annealing with a YAG:Nd laser $(\lambda=1064 \mathrm{~nm}$ and $\lambda=532 \mathrm{~nm})$, with a power density in the range of $10-112 \mathrm{MW} \mathrm{cm}^{-2}\left(100-1120 \mathrm{~mJ} \mathrm{~cm} \mathrm{~cm}^{-2}\right.$ for a $10 \mathrm{~ns}$ pulse duration). After irradiation with a wavelength of $1064 \mathrm{~nm}$ and power density of $14 \mathrm{MW} \mathrm{cm} \mathrm{cm}^{-2}$, the formation of $5 \mathrm{~nm}$ high nanoislands was observed. In the case of $\lambda=532 \mathrm{~nm}$, the height of nanoislands was multiplied, reaching a value of $50 \mathrm{~nm}$, whereas a decrease in optical transmittance was reported. Therefore, the suggested theoretical model regarding a linear temperature-gradient dependence of the laser intensity, also confirmed by the aforementioned experimental data, enabled a pre-estimation of the localized $\mathrm{SiO}_{2}$ nanostructure formation. ${ }^{[161]}$

The investigation of sol-gel $\mathrm{HfO}_{2}$ film properties under $\mathrm{XeCl}$ laser annealing was employed by Teodorescu et al., after their deposition onto a $\mathrm{Si} / \mathrm{SiO}_{2}$ structure. The number of pulses ranged from 100 to 10000 , in conformity with various laser fluences $\left(30-120 \mathrm{~mJ} \mathrm{~cm} \mathrm{~cm}^{-2}\right.$ ). Despite the absence of changes after a small number of pulses in a medium fluence $\left(65 \mathrm{~mJ} \mathrm{~cm} \mathrm{~cm}^{-2}\right)$, cross-sectional transmission electron microscopy (XTEM) analysis of the $\mathrm{Si} / \mathrm{SiO}_{2} / \mathrm{HfO}_{2}$ structure confirmed the presence of an intermediate $\mathrm{Si}_{1-x} \mathrm{Hf}_{x} \mathrm{O}_{2}$ layer after 10000 pulses at $80 \mathrm{~mJ} \mathrm{~cm}{ }^{-2}$, accompanied by film densification. Apparently, the intense laser annealing led to an increase in $\mathrm{HfO}_{2}$ dielectric constant $(\approx 25)$, though the additional contribution of the $\mathrm{Si}_{1-x} \mathrm{Hf}_{x} \mathrm{O}_{2}$ layer into the increased dielectric constant remained unclear. A subsequent examination on the $\mathrm{HfO}_{2}$ optical properties indicated its transparency in $\mathrm{XeCl}$ wavelength $(\lambda=308 \mathrm{~nm})$, resulting in the assumption of heated $\mathrm{Si}$ (high absorption) acting as the main contributor into $\mathrm{HfO}_{2}$ heating. The dielectric measurements after various fluences revealed a reduced dielectric constant following low fluences, mainly due to the incomplete organic residue removal. [153]

\subsubsection{Metal-Oxide Conductors}

An extensive body of work on LA of conductive metal oxides, mainly used as transparent electrodes, has been reported. ${ }^{[125-127,129,162-172]}$ Amongst them, the influence of laser treatment on commercially available fluorine-doped tin oxide (FTO) has been thoroughly investigated by Li et al. After reporting on the optoelectronic response of laser treated $\mathrm{Pt} /$ FTO $^{[172]}$ and Ni/FTO thin films, ${ }^{[162]}$ their work focused on the effect of ultrasonic vibration during laser irradiation. In detail, they reportedly examined the crystallinity dependence on the frequency and power of the ultrasonic vibration during LA $\left(\mathrm{Nd}: \mathrm{YVO}_{4}, \lambda=532 \mathrm{~nm}\right.$, laser fluence at $600 \mathrm{~mJ} \mathrm{~cm}{ }^{-2}$ ), leading to the formation of smoother and highly oriented crystallized FTO films (XRD analysis) in the case of ultrasonication at $48 \mathrm{kHz}$ and $300 \mathrm{~W}$. This enhancement was mainly attributed to the elimination of particle clusters during ultrasonication and LA, forming smaller and more compact FTO crystallites, therefore leading to a denser and smoother film. This densification resulted in transparency increase, accompanied by a slight decrease in Sheet Resistance values (9.3-8.8 $\Omega \square^{-1}$ ), mainly attributed to the increased film compactness (reduced porosity). ${ }^{[163]}$

Moreover, various studies regarding patterning of sputtered ITO films, ${ }^{[129]}$ sintering of spin-coated ITO nanoparticles ${ }^{[127]}$ and enhancement of sputtered ITO electronic properties after beam-shaped laser annealing ${ }^{[164]}$ have been reported. To this end, pioneering work on the optoelectronic properties of solgel ITO films after ns KrF laser treatment was reported by Noh et al. ${ }^{[165]}$ After irradiation with laser fluences varying from 50 to $240 \mathrm{~mJ} \mathrm{~cm}{ }^{-2}$, a remarkable difference in the optical conductivity spectra was observed. Specifically, an increase in the absorption spectra was reported in both the low and high energy regions and was attributed to the enhancement of charge carrier concentration/transport within the ITO layer. A gradual increase of the optical bandgap was also reported and was attributed to the enhanced crystallization of ITO, along with an increase in the interband transition energy caused by the band filling due to increased carrier concentration.

Kim et al. reported an in-depth study of the optoelectronic properties of LA sol-gel ITO films together with an extensive examination of the electrical and chemical properties of the formed layers. ${ }^{[126]}$ Hall effect measurements revealed a decrease in the resistivity of the laser-treated ITO films, an outcome consistent with the previous ellipsometry results of Noh et al. ${ }^{[165]}$ The remarkable decline of resistivity values (from 1663 to $5.75 \mathrm{~m} \Omega \mathrm{cm}$ ) was attributed primarily to the increased carrier density upon LA. The deconvolution of the X-ray photoelectron spectroscopy (XPS) peaks corresponding to $\mathrm{C}$, O, In and $\mathrm{Sn}$, interestingly confirmed the crystallinity enhancement of the ITO films with increasing

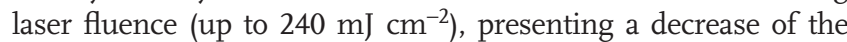
$\mathrm{O}_{\text {III }}$ and $\mathrm{In}_{\text {II }}$ peaks along with an increase of $\mathrm{Sn}_{\text {II }}$ peak, corresponding respectively to $-\mathrm{OH}$ atoms, In atoms bonded with a small amount of electrons (amorphous phase), and $\mathrm{SnO}_{2}$ phase. These results, combined with the decrease (increase) in $\mathrm{C}$ (oxygen and metals) concentration, as well as the rise in XRD peak intensity and the formation of larger crystallites (observed by TEM analysis) after laser treatment in increasing fluences, confirmed the gradual transition from the organometallic precursor into a highly crystalline ITO layer. 


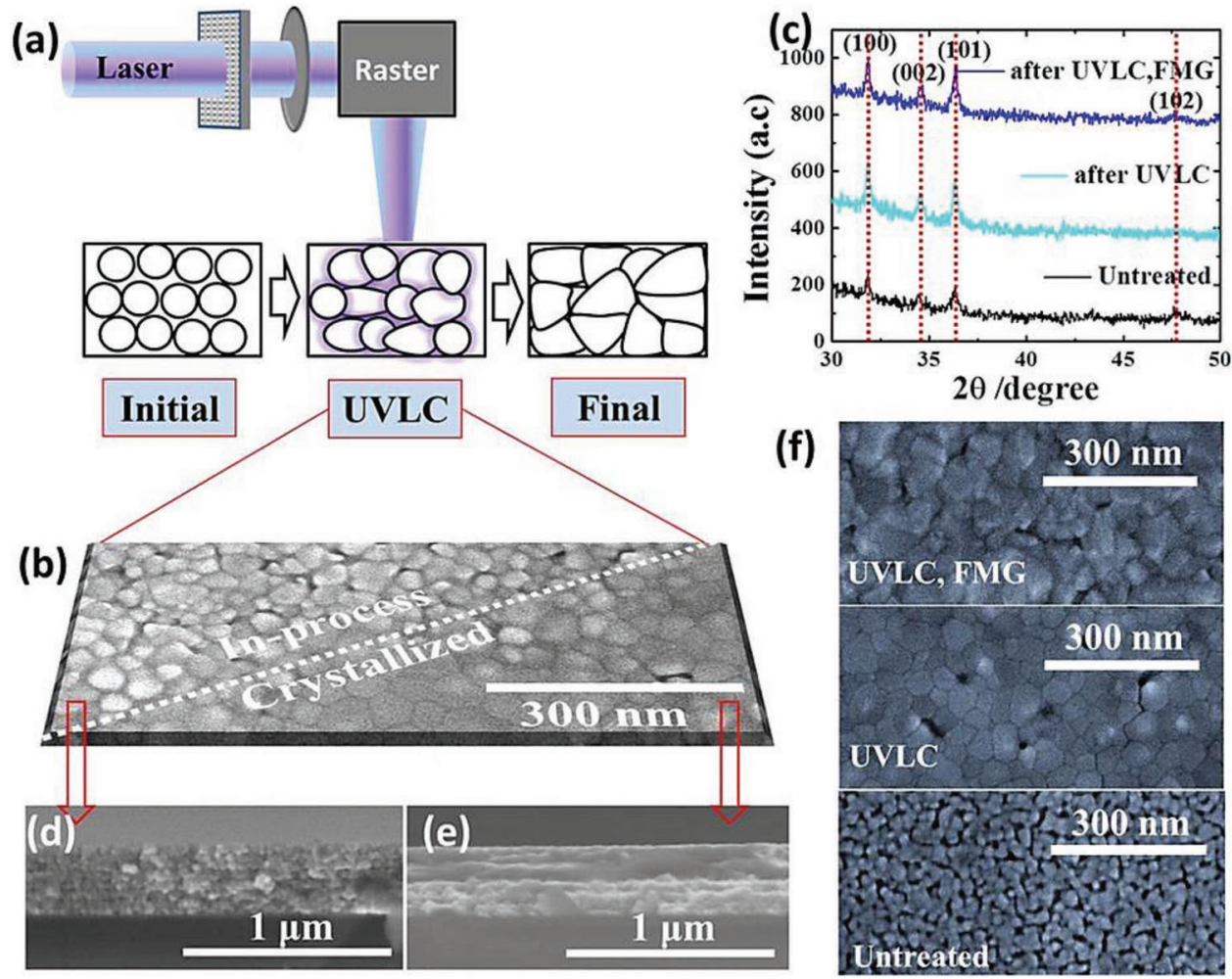

Figure 9. a) Proposed laser annealing scheme for AZO films. b) Plane-view SEM image of AZO film under ongoing LA. c) XRD analysis of the laser irradiated AZO films. Cross-section SEM image of AZO film d) before and e) after LA. f) Plain view FESEM image of AZO films before and after laser treatment. Reproduced under the terms of the Creative Commons Attribution 4.0 International License. ${ }^{[125]}$ Copyright 2015, Springer Nature.

Nian et al. investigated the effect of $\mathrm{KrF}$ laser annealing on sol-gel aluminum-doped zinc-oxide (AZO) thin films. ${ }^{[125]}$ At first, a localized temperature increase simulation was carried out via the software COMSOL Multiphysics, according to which, KrF laser irradiation led to the formation of "hot spots" between AZO as-deposited nanoparticles. In order to further examine this heat transfer mechanism between nanoparticles, Molecular Dynamic Simulation was conducted. According to their calculations, the NPs coalesce together, forming "patch"like phases with interconnect NPs. The closely packed NPs resulted in TFTs with enhanced electron mobility.

The agreement of the macroscale COMSOL simulations with the nanoscale Molecular Dynamic simulations suggested the three steps formation mechanism for the AZO layers upon $\mathrm{KrF}$ laser irradiation shown in Figure 9a. First, solution processing leads to the formation of AZO NPs assemblies, followed by the emergence of "hot-spots" between NPs after irradiation. The latter effect initiates their partial melting which in turn enables the particles to coalesce. The result is the formation of large-sized domains of AZO (Figure 9b). This presence of large crystalline grains was confirmed by XRD analysis (Figure 9c). Figure 9d,e shows the cross-sectional field emission scanning electron microscopy (FESEM) images before and after KrF irradiation, which clearly depict the induced crystallization, along with the elimination of internal gaps, as also seen in Figure of. The resistivity values $\rho_{\text {hall }}$ after $\mathrm{KrF}$ irradiation presented a significant decrease, (from $2.28 \times 10^{-3}$ to $2.3 \times 0^{-3} \Omega \mathrm{cm}$ after
150 pulses), with a high mobility value of $18.1 \mathrm{~cm}^{2} \mathrm{~V}^{-1} \mathrm{~s}^{-1}$. A further investigation regarding the factors leading to the reported mobility enhancement was employed, especially after an additional forming gas treatment (FMG) (under $400{ }^{\circ} \mathrm{C}$ for $1 \mathrm{~h}$ ). According to the authors, $\mathrm{KrF}$ laser irradiation dissociates weak $\mathrm{Zn}-\mathrm{O}$ bonds, forming oxygen vacancies, while inducing desorption of oxygen species, leading to free carrier release from trap states. The latter process is further enhanced by FMG, which also contributes to the formation of oxygen vacancies-confirmed by XPS measurements. ${ }^{[125]}$

A few years later, El Hamali et al. ${ }^{[166]}$ successfully demonstrated an enhancement in the optoelectronic properties of radio frequency (RF) sputtered AZO films, after excimer laser annealing (ELA) treatment with a $\mathrm{KrF}$ excimer laser. Specifically, the examination of various deposition parameters such as oxygen concentration, RF power and sputtering pressure was initially conducted, aiming to provide information regarding their link with the resulted film resistivity. Interestingly, both free carrier density and Hall mobility values were found to increase with respect to RF power increase and pressure decrease. The formed layers were found to exhibit enhanced crystallinity and reduced concentration of electron traps. Following the optimization of deposition parameters, the effect of LA on the electrical resistivity was studied. After a single pulse of up to $125 \mathrm{~mJ} \mathrm{~cm}{ }^{-2}$, a decrease in resistivity was obtained, whereas, after five pulses, resistivity values were $50 \%$ lower compared to as-deposited AZO films, reaching $5 \times 10^{-4} \Omega \mathrm{cm}$. 
It is worth mentioning that LA in higher fluences resulted in ablation phenomena. The deconvolution of O 1s XPS peak revealed a decrease in oxygen vacancies as well as $-\mathrm{OH}$ related species (which act as trapping and scattering centres), thus explaining the enhancement of the electron concentration and mobility. It was suggested that this free carrier concentration increase could originate from the $\mathrm{Al}$ activation in $\mathrm{ZnO}$ matrix, following LA. In addition, the reduction in film roughness, along with a decrease in structural defects after LA, led to an enhancement in visible transparency (reaching $90 \%$ ), accompanied by an increase in energy bandgap $E_{\mathrm{g}}$.

\subsubsection{Metal-Oxide Semiconductors}

The effect of LA on metal-oxide semiconducting films has been gathering increasing attention, leading to numerous published studies of their mechanical and optoelectronic properties as well as combinations of various fabrication approaches and materials. ${ }^{[122,123,173-182]}$ Tsay et al. ${ }^{[182]}$ successfully fabricated sol-gel IGZO films and utilized $\mathrm{KrF}$ excimer laser annealing in order to examine its impact on the optical, morphological and electrical properties of the layers. In comparison with thermal annealing, laser annealed IGZO presented spheroidal grain morphologies that led to the formation of densified, uniform and transparent films. Regarding their electrical response,

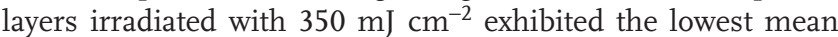
resistivity values $\left(4.48 \times 10^{3} \Omega \mathrm{cm}\right)$, together with the highest electron mobility $\left(5.26 \mathrm{~cm}^{2} \mathrm{~V}^{-1} \mathrm{~s}^{-1}\right)$. Kim et al. ${ }^{[122]}$ investigated the effect of $\mathrm{KrF}(248 \mathrm{~nm})$ excimer laser annealing on sol-gel $\mathrm{ZnO}$ films, and showed that laser treatment led to the crystallization of the initially amorphous film, with subsequent decrease of the blue-green PL peak at $3.26 \mathrm{eV}$.

An interesting study on the effects of $\mathrm{XeCl}$ excimer laser annealing on chemical bath deposited $\mathrm{ZnO}$ nanorods, using Si and PI substrates, was employed by Fiaschi et al. ${ }^{[123]}$ In the case of Si substrate (Figure 10a), the operational window of energy densities was located at $220-465 \mathrm{~mJ} \mathrm{~cm}^{-2}$. A gradual transformation from $\mathrm{ZnO}$ nanorods to spherical structures was observed while increasing the beam fluence. The low thermal conductivity of PI, though, led to a significantly narrower operational window, as in $200 \mathrm{~mJ} \mathrm{~cm} \mathrm{~cm}^{-2}$ the $\mathrm{ZnO}$ nanorods were transformed into a continuous layer with the presence of holes indicating the induced ablation (Figure 10b). PL analysis on the Si substrate-based films showed an enhancement of the UV emission peak, whereas the peak corresponding to the presence of defects (at $570 \mathrm{~nm}$ ) decreased. On the contrary, PL analysis of the $\mathrm{ZnO}$ films grown on PI substrates resulted to a reduction of the UV peak with increasing fluence, indicating the occurrence of ablation.

The effect of LA on the PL of ZnO has been extensively investigated by Tsakonas et al. ${ }^{[174]}$ In this study the authors presented results on the $\mathrm{KrF}$ laser annealing of sputtered $\mathrm{ZnO}$ films on silicon and its effects on intrinsic PL characteristics. Processing with a single pulse at $235 \mathrm{~mJ} \mathrm{~cm} \mathrm{c}^{-2}$ it was possible to create visible luminescence (deep level emission (DLE), associated to defects in the film), where the as-deposited films showed no PL whatsoever. Increasing the number of pulses or alternatively increasing the fluence to $295 \mathrm{~mJ} \mathrm{~cm}^{-2}$ (single pulse) resulted in strong and sharp UV photoluminescence at $381 \mathrm{~nm}$ (near band edge (NBE) emission, associated to highquality crystalline $\mathrm{ZnO}$ ).

Nedyalkov et al. ${ }^{[173]}$ investigated the effect of LA on Pulsed Laser Ablated $\mathrm{ZnO}$ films on Ta substrates, after altering the fluence as well as the number of the ns pulses. Under $50-80 \mathrm{~mJ} \mathrm{~cm}{ }^{-2}$ laser irradiation, the emergence of melting spots that led to irregular droplet formation was observed. The phenomenon was attributed to initial film defect points. Interestingly, the film behavior after exceeding the melting threshold showed remarkable differences, since, for fluences over $150 \mathrm{~mJ} \mathrm{~cm}{ }^{-2}$, a homogenous formation of large, wellshaped, closely packed $\mathrm{ZnO}$ nanoparticles was reported. In order to enlighten the mechanism of melting and re-solidification of $\mathrm{ZnO}$ layers, computer simulations of the heating and (a)

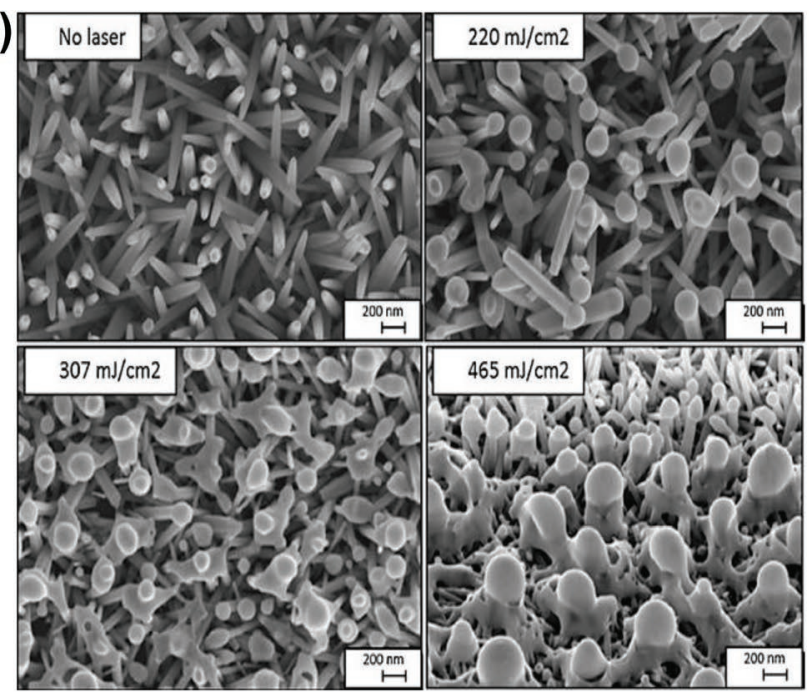

(b)
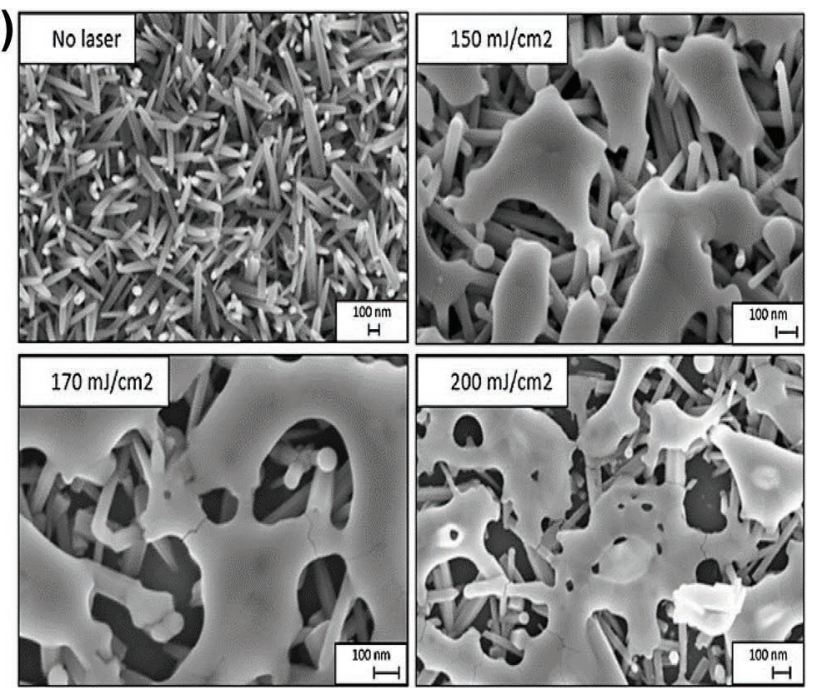

Figure 10. SEM images of a) as-grown and laser annealed ZnO nanorods at 220, 307, and $465 \mathrm{~mJ} \mathrm{~cm}^{-2}$, respectively, on Si substrate. b) As-grown and laser annealed ZnO nanorods at 150, 170, and $200 \mathrm{~mJ} \mathrm{~cm}{ }^{-2}$, respectively, on PI substrate. Reproduced with permission. ${ }^{[123]}$ Copyright 2018, Elsevier. 
cooling, based on the heat diffusion model, were performed. The obtained results indicated rapid heating (up to $2800 \mathrm{~K}$ ) and cooling of the $\mathrm{ZnO}$ film at high fluences, leading to its homogenous decomposition. The high cooling rates were mainly attributed to the metal substrate, inducing a cooling rate of $100 \mathrm{~K} \mathrm{~s}^{-1}$.

\subsection{Application of Laser Annealing in Metal-Oxide TFT Fabrication}

The use of LA in TFT manufacturing has become well established in recent years, linking a plethora of new materials with innovative processing paradigms. Amongst the different metal-oxide semiconductors, IGZO has been well studied in the context of postdeposition laser treatment. A series of pioneering studies on sputtered IGZO TFTs and the impact of $\mathrm{XeCl}$ excimer laser irradiation (308 $\mathrm{nm}$ ) were reported by Nakata et al. ${ }^{[183,184]}$ In addition to their studies on laser annealed IGZO TFTs on glass and polymer substrates, their work also included an investigation of the temperature gradient during laser annealing. Their calculations showed that LA (fluence $150 \mathrm{~mJ} \mathrm{~cm}{ }^{-2}$ ) of the $20 \mathrm{~nm}$ thick IGZO film induced a dramatic $1500{ }^{\circ} \mathrm{C}$ temperature increase in the semiconductor but only 12 and $6{ }^{\circ} \mathrm{C}$ change for the polymer and glass substrates, respectively. Following the confirmation of no significant temperature rise in the substrate, a temperature profile along the IGZO thickness was calculated, after depositing an intermediate $\mathrm{SiO}_{2}$ buffer layer of various thicknesses for polymer substrate protection. As suggested, the optimized thickness of $\mathrm{SiO}_{2}$ buffer layer was $600 \mathrm{~nm}$, below which IGZO films would peel

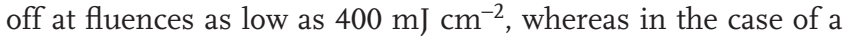
$600 \mathrm{~nm}$ thick $\mathrm{SiO}_{2}$ no pealing was observed, even after irradiation at $600 \mathrm{~mJ} \mathrm{~cm}{ }^{-2}$. Analysis of the crystallinity of the IGZO films by XRD revealed that only laser fluences of $>400 \mathrm{~mJ} \mathrm{~cm}{ }^{-2}$ resulted in melting and recrystallization of IGZO films. TFTs irradiated with $170 \mathrm{~mJ} \mathrm{\textrm {cm } ^ { - 2 }}$ exhibited enhanced electron mobility of $15.6 \mathrm{~cm}^{2} \mathrm{~V}^{-1} \mathrm{~s}^{-1}$ and an increased $(10 \times)$ channel ON current as compared to untreated IGZO devices. The performance enhancement was attributed mainly to a reduction in the electron trap density upon LA for fluences $<200 \mathrm{~mJ} \mathrm{~cm}{ }^{-2}$. Particular attention was paid on the peeling-off phenomenon of IGZO films in the case of polymer substrates. The correlation between the buffer layer thickness and the maximum laser fluence before the peeling-off stage was examined and suggested that observed stresses originated from the differences in the thermal expansion coefficients between the different materials (i.e., IGZO/substrate). In the case of TFTs, the OFF current increase observed for high fluences LA was also investigated and attributed to the increased electron density. ${ }^{[185]}$

Zan et al. ${ }^{[77]}$ performed a comparative study between Nd:YAG (266 nm) laser-treated and UV lamp-treated IGZO TFTs. According to the authors, the lower critical energy is needed for boosting of the transistor performance (as compared to the aforementioned Nakata's studies) was attributed primarily to the enhanced absorption of IGZO film at $266 \mathrm{~nm}$ compared to $\mathrm{XeCl}$ laser irradiation. This enhancement in TFT performance was attributed to the relaxation of the a-IGZO phase after laser treatment, in addition to the removal of weakly bonded oxygen atoms (i.e., generation of oxygen vacancies). Overall, IGZO TFTs irradiated with UV light for 30 min exhibited enhanced electron mobility reaching a maximum value of $3 \mathrm{~cm}^{2} \mathrm{~V}^{-1} \mathrm{~s}^{-1}$.

Besides magnetron sputtering, numerous other techniques have been reportedly utilized for the manufacturing of IGZO TFTs and successfully combined with LA treatment including, spin-coating ${ }^{[86]}$ and atmospheric pressure plasma jet (APPJ) techniques. ${ }^{[187]} \mathrm{Wu}$ et al., ${ }^{[188]}$ employed atmospheric pressure plasma-enhanced chemical vapor deposition (PE-CVD) technique together with $\mathrm{KrF}$ laser treatment to fabricate IGZO TFTs based on $\mathrm{LaAl}_{2} \mathrm{O}_{3} / \mathrm{ZrO}_{2}$ as the gate dielectric. Laser pulses of $50-200 \mathrm{~mJ} \mathrm{~cm} \mathrm{~cm}^{-2}$ were used in order to observe significant changes in the transistors' electrical performance. A large increase in Hall mobility of the IGZO layers with increasing laser fluence was observed. An enhancement in channel ON current and an increase in the electron mobility to $10 \mathrm{~cm}^{2} \mathrm{~V}^{-1} \mathrm{~s}^{-1}$ was reported upon treatment of the TFTs with $150 \mathrm{~mJ} \mathrm{~cm}{ }^{-2}$, i.e., $\times 20$ higher value than that measured for the pristine devices $\left(0.5 \mathrm{~cm}^{2} \mathrm{~V}^{-1} \mathrm{~s}^{-1}\right)$. The improvement was accompanied by a decrease in $V_{\mathrm{TH}}(0.3 \mathrm{~V})$, SS $\left(0.15 \mathrm{~V} \mathrm{dec}^{-1}\right)$ and an increase of the channel current ON/OFF ratio to $>10^{7}$. Treatment of the IGZO TFTs with higher laser fluences resulted in increased channel conductivity and electron mobility but was accompanied by a dramatic deterioration of the channel ON/OFF ratio, SS and $V_{\mathrm{TH}}$.

The application of ns pulsed laser treatment has also been expanded to $\mathrm{ZnO}$, leading to the realization of TFTs via different deposition techniques, including sputtering ${ }^{[189]}$ and dropcasting of ZnO NPs. ${ }^{[18]}$ Maeng et al. reportedly manufactured $\mathrm{ZnO}$ nanowire-based TFTs and investigated the influence of $\mathrm{KrF}$ excimer laser irradiation under an inert atmosphere. Laser treatment with fluences of $50-200 \mathrm{~mJ} \mathrm{~cm}{ }^{-2}$ was found to alter

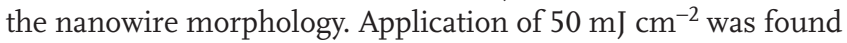
to partially melt the top surface of the $\mathrm{ZnO}$ nanowires where an excess of $\mathrm{Zn}$ was identified due to the lack of oxygen in the $\mathrm{N}_{2}$ rich atmosphere where the laser annealing step was conducted. As fluence increased to 100 and $200 \mathrm{~mJ} \mathrm{~cm} \mathrm{~cm}^{-2}$, melting and breaking of the $\mathrm{ZnO}$ nanowires was observed, resulting in a decrease of the PL emission along with a decrease in the intensity of the XRD peak. XPS analysis revealed a shift in $\mathrm{Zn} 2 \mathrm{p}$ and $O$ 1s peaks toward higher energies upon laser annealing, indicating an increase in oxygen vacancies. The influence of the laser treatment step on the $\mathrm{ZnO}$ nanowire TFTs was also found to be dramatic. Specifically, the formation of oxygen vacancies resulted in a higher carrier density, a deterioration in SS and a shift of $V_{\mathrm{TH}}$ toward more negative voltages, which is indicative of increased electron concentration in the channel. Devices treated with $100 \mathrm{~mJ} \mathrm{~cm} \mathrm{~cm}^{-2}$ laser fluence exhibited the maximum electron mobility value of $27.9 \mathrm{~cm}^{2} \mathrm{~V}^{-1} \mathrm{~s}^{-1}$. [190]

Recently, Oh et al. ${ }^{[191]}$ studied the influence of $\mathrm{KrF}$ excimer annealing onto PE-CVD ZnO TFTs using fluences of 200 and $300 \mathrm{~mJ} \mathrm{~cm}{ }^{-2}$. At first, a thorough examination of the alternation of $\mathrm{ZnO}$ layer properties following laser irradiation was conducted. Rapid melting and recrystallization of $\mathrm{ZnO}$ films using fluences up to $200 \mathrm{~mJ} \mathrm{~cm}{ }^{-2}$ were shown to lead to crystallinity enhancement (Figure 11a), accompanied by a decrease in layer surface roughness. Nevertheless, the transition into higher fluences $\left.(300 \mathrm{~mJ} \mathrm{~cm})^{-2}\right)$ resulted in layer deterioration, inducing the formation of grain boundaries and cracks, a phenomenon reinforced by 
(a)

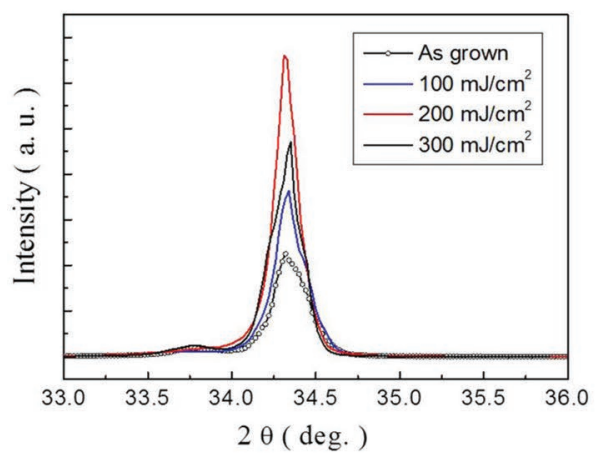

(b)

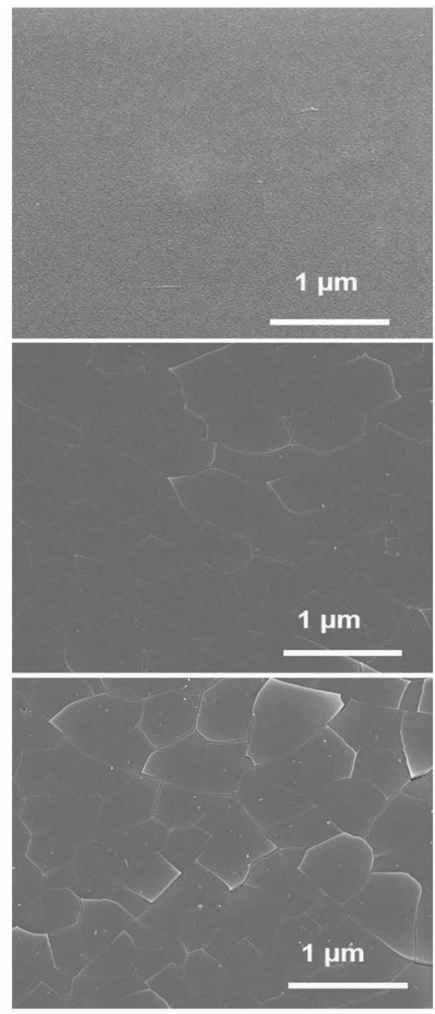

(c)
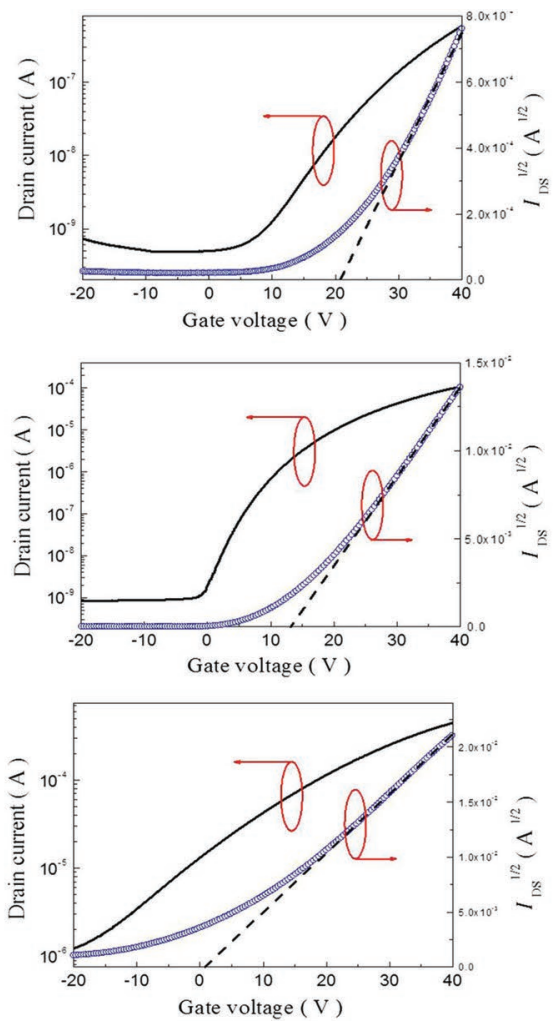

Figure 11. a) XRD peak intensities of the (0002) rocking curves of $\mathrm{ZnO}$ thin films before and after laser annealing with various laser fluences. b) FESEM surface images of $\mathrm{ZnO}$ laser annealed thin films at laser fluences of as-grown (top), $200 \mathrm{~mJ} \mathrm{~cm} \mathrm{~cm}^{-2}$ (middle), and $300 \mathrm{~mJ} \mathrm{~cm}$ (bottom). c) Transfer characteristics of the laser annealed ZnO TFTs at laser fluences of as-grown (top), $200 \mathrm{~m} / \mathrm{cm}^{-2}$ (middle), and $\left.300 \mathrm{~m}\right) \mathrm{cm}^{-2}$ (bottom). Reproduced with permission. ${ }^{[191]}$ Copyright 2019, The Minerals, Metals \& Materials Society, published by Springer Nature.

the subsequent increase in roughness. The aforementioned results were confirmed by XRD analysis. Besides, a detailed investigation of the crystallization mechanism was conducted. Laser irradiation was shown to enhance re-crystallization in the lateral direction due to energy propagation in this direction. This characteristic lateral growth resulted in the formation of large grains (Figure 11b) and a higher electron field-effect mobility. The TFT output characteristics upon laser treatment with 200 and $300 \mathrm{~mJ} \mathrm{~cm}{ }^{-2}$, indicated a current-crowding effect in the case of the nonannealed TFTs, whereas in laser annealed devices this phenomenon was not observed, mainly due to the enhanced Ohmic nature of the injecting contact. Moreover, a clear shift of $V_{\mathrm{TH}}$ toward zero volts, from 25.5 to $1.1 \mathrm{~V}$ (Figure 11c), was observed upon laser irradiation $\left(300 \mathrm{~mJ} \mathrm{~cm}^{-2}\right)$, accompanied by a mobility increase to $19.27 \mathrm{~cm}^{2} \mathrm{~V}^{-1} \mathrm{~s}^{-1}$.

Dellis et al. ${ }^{[9]}$ investigated the influence of ELA on solution processed $\mathrm{In}_{2} \mathrm{O}_{3}$ TFTs. At first, $\mathrm{In}_{2} \mathrm{O}_{3}$ precursor was spun onto $400 \mathrm{~nm} \mathrm{Si} / \mathrm{SiO}_{2}$ substrates. Various laser fluences, ranging from 300 to $450 \mathrm{~mJ} \mathrm{~cm} \mathrm{~cm}^{-2}$ irradiated the as-spun samples, converting the precursor layer into high-quality $\mathrm{In}_{2} \mathrm{O}_{3}$ films. Figure 12a shows the TFT electrical characteristics after laser annealing with 10 pulses at energy fluence $300 \mathrm{~mJ} \mathrm{~cm}{ }^{-2}$. The electrical performance of the TFTs was remarkably different with respect to the increase of the laser fluence and the number of pulses (ranging from 1 to 10). Specifically, the mobility values measured in the ELA treated devices ranged from 9 to $13 \mathrm{~cm}^{2} \mathrm{~V}^{-1} \mathrm{~s}^{-1}$ (the highest mobility was reported for laser fluence of $300 \mathrm{~mJ} \mathrm{~cm}{ }^{-2}$ ), while the $V_{\mathrm{TH}}$ increased with increasing energy fluence. In order to shift $V_{\mathrm{TH}}$ closer to zero, a mild TA step $\left(100{ }^{\circ} \mathrm{C}\right.$ for $\left.60 \mathrm{~min}\right)$ was conducted. This treatment did not influence the channel ON/OFF ratio $\left(>10^{5}\right)$, although a slight decrease of the mobility values $\left(6-10 \mathrm{~cm}^{2} \mathrm{~V}^{-1} \mathrm{~s}^{-1}\right)$ was observed (Figure 12b). In order to examine the conversion mechanism during ELA treatment, XPS analysis was conducted. The deconvolution of the O 1s XPS peak revealed a remarkable increase in the $[\mathrm{In}-\mathrm{O}] /[\mathrm{In}-\mathrm{OH}]$ bond ratio following laser treatment (1.10 instead of 0.64 of the thermally annealed films), which was further boosted to 1.27 after thermal annealing.

In addition to the aforementioned studies focusing on the semiconductor layer, LA has also been employed for selective patterning of highly conductive regions, which can be utilized as a part of the S/D electrodes. Nakata et al. ${ }^{[192]}$ reported the fabrication of self-aligned IGZO TFTs using a XeCl laser. After the deposition of an $\mathrm{Al}$ gate electrode, $\mathrm{SiO}_{2}$ dielectric and IGZO semiconductor layer on a glass substrate, back-side irradiation was utilized to selectively anneal the IGZO regions that did not overlap with the gate electrode. This postdeposition irradiation decreased the sheet resistance of IGZO from $10^{7}$ to $1 \mathrm{k} \Omega \square^{-1}$. For laser fluences higher than $125 \mathrm{~mJ} \mathrm{~cm}^{-2}$ the sheet resistance remained low exhibiting the same values compared to front side irradiated samples. Following the irradiation, Mo S/D electrodes were deposited by avoiding parasitic overlapping with the gate electrode. Irradiated TFTs exhibited higher electron mobility $\left(8.2 \mathrm{~cm}^{2} \mathrm{~V}^{-1} \mathrm{~s}^{-1}\right)$ than nonirradiated transistors. Moreover, 
(a)

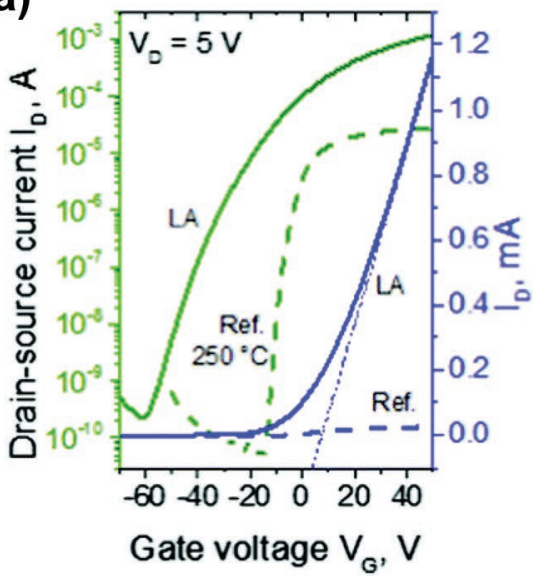

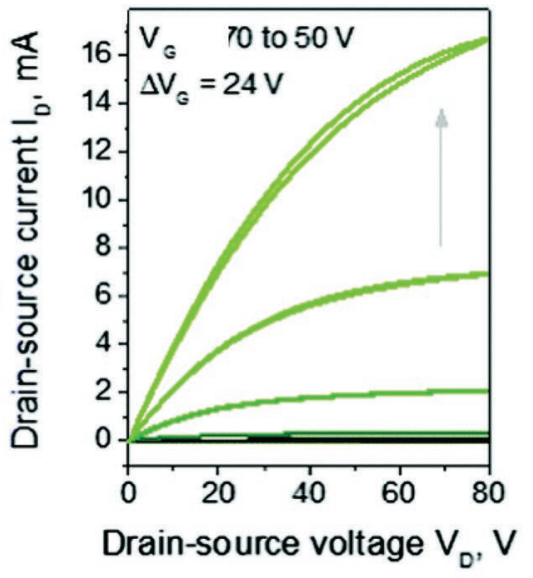

Figure 12. a) Electrical characteristics of ELA treated $\ln _{2} \mathrm{O}_{3}$ TFTs (10 pulses at energy fluence of $300 \mathrm{~mJ} \mathrm{~cm} \mathrm{~cm}^{-2}$ ) after the mild thermal annealing b) $V_{T H}$ and mobility values of ELA treated $\operatorname{In}_{2} \mathrm{O}_{3}$ TFTs before and after mild thermal annealing. Reproduced with permission ref. [99]. Copyright 2017, The Royal Society of Chemistry.

the parasitic capacitance of self-aligned TFTs was investigated reaching values of $0.1 \mathrm{fF} \mu \mathrm{m}^{-1}$, an order of magnitude lower as

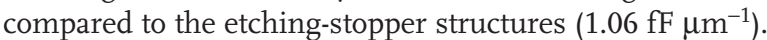

The impact of channel thickness variation in self-aligned gate IGZO TFTs was also studied a few years later. ${ }^{[193]}$ The sheet resistance of 30, 50, and $100 \mathrm{~nm}$ thick IGZO films on glass substrate was examined after backside irradiation with a $\mathrm{XeCl}$ laser (Figure 13a). Interestingly, the sheet resistance was found to decrease with increasing IGZO thickness (Figure 13b). In addition, the laser fluence at which sheet resistance remained (a)

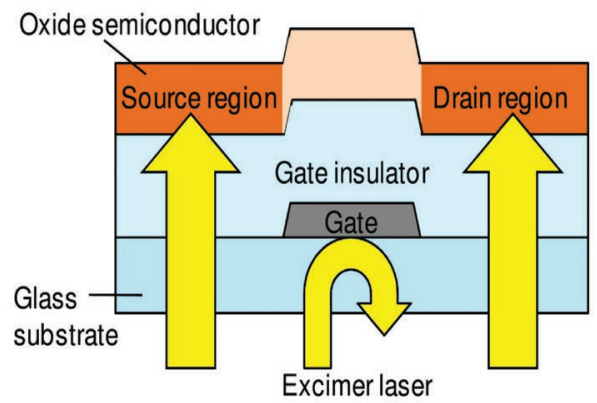

(b)

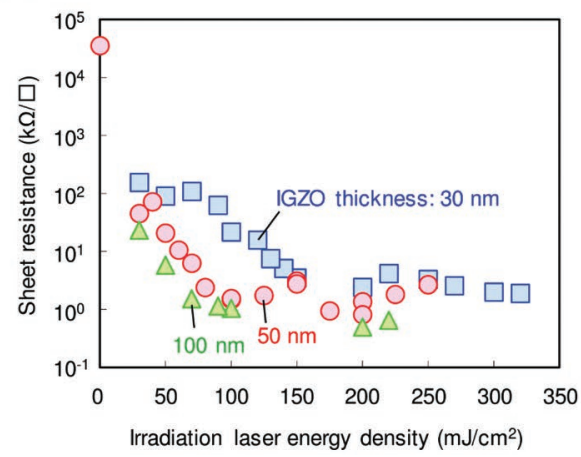

(c)
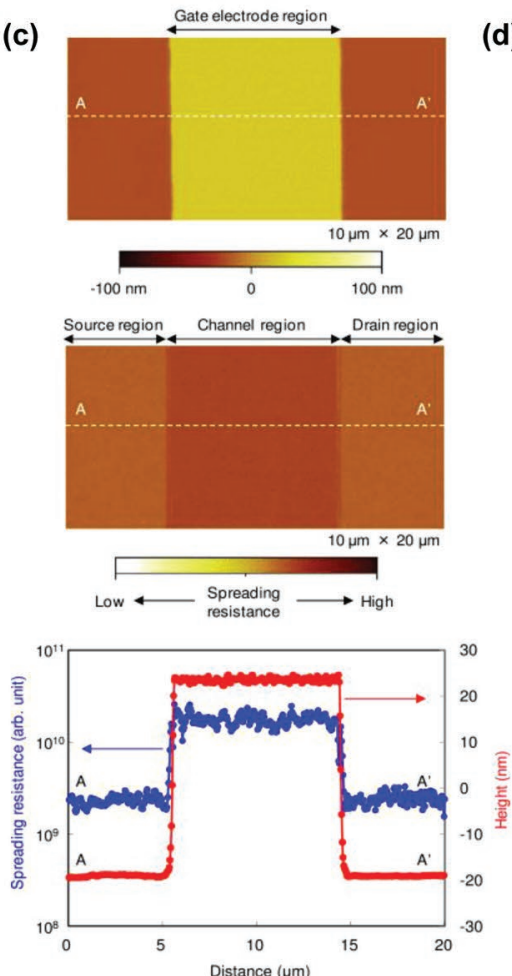

(d)
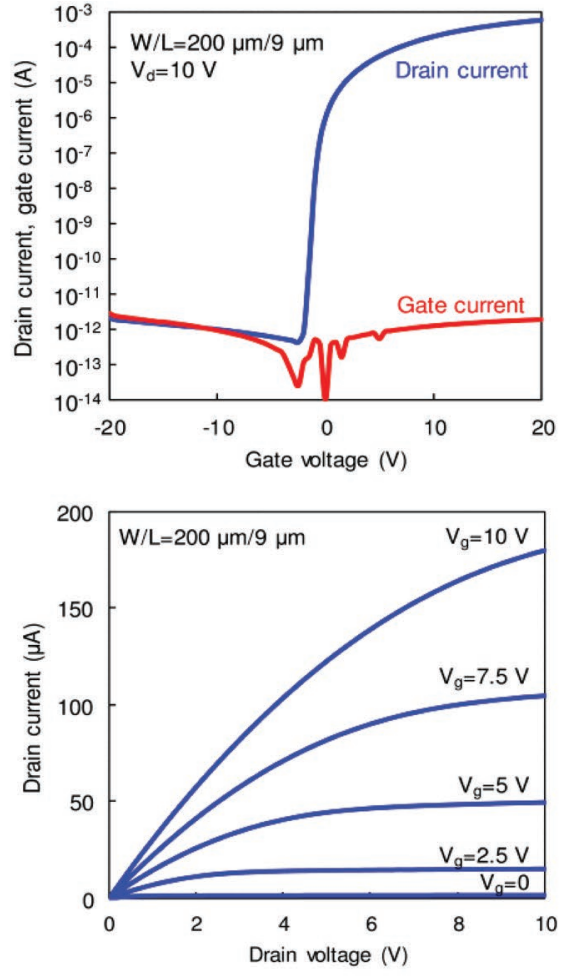

Figure 13. a) Schematic image of self-aligned bottom-gate, bottom-contact ICZO-TFT fabrication by backside irradiation of XeCl laser. b) Sheet resistance values of various IGZO thicknesses, following backside irradiation with a XeCl laser. c) AFM height image, SSRM spreading resistance image, and spreading resistance and height profiles of IGZO film atop the Al gate electrode in self-aligned gate TFTs. d) Transfer and output characteristics of the self-aligned IGZO TFTs. Reproduced with permission. ${ }^{[193]}$ Copyright 2017, IEEE. 
$>5 \mathrm{k} \Omega \square^{-1}$ also decreased for thicker IGZO layers. This behavior was attributed to the decreased heat capacity of thinner IGZO films. Scanning spreading resistance microscopy (SSRM) was utilized in order to measure the resistance and height distribution of the IGZO layer (Figure 13c), after laser backside irradiation. The formation of a high central resistance region and two low resistance S/D regions were observed. Atomic force microscopy (AFM) images (Figure 13c) confirmed the existence of these regions and highlighted backside irradiation as an effective way of controlling the operating characteristics of the TFTs. Figure 13d shows the transfer and output characteristics of the self-aligned gate IGZO-TFTs. The devices exhibited electron mobility of $9.4 \mathrm{~cm}^{2} \mathrm{~V}^{-1} \mathrm{~s}^{-1}, V_{\mathrm{TH}}=-0.8 \mathrm{~V}$ and $\mathrm{SS}=0.22 \mathrm{~V} \mathrm{dec}^{-1}$, further highlighting the potential of the method.

The impact of laser irradiation following the completion of the TFT fabrication has also been reported. The influence of $\mathrm{XeCl}$ as well as $\mathrm{KrF}$ excimer laser annealing on the electrical performance of sputtered $\alpha$-IGZO TFTs passivated with polysilsesquioxane (PSQ) was examined by Bermundo et al. ${ }^{[194]}$ Considering the high transparency of the PSQ layer at the laser's wavelengths, their irradiation primarily affected the $\mathrm{Pt}$ and Mo electrodes deposited atop the IGZO layer. The high channel off current and the overall degradation in device operation of the pristine devices was remarkably improved after LA (Figure 14a), exhibiting mobility values of $12.5 \mathrm{~cm}^{2} \mathrm{~V}^{-1} \mathrm{~s}^{-1}$, $V_{\mathrm{TH}}=3 \mathrm{~V}$ and on-off ratios of $1.1 \times 10^{10}$. A further investigation attempting to link the heat propagation mechanism to device dimensions was conducted, revealing a dramatic shrinkage in the effective channel width from 90 to $5 \mu \mathrm{m}$ (Figure 14a). According to $2 \mathrm{D}$ heat propagation simulations, a sharp increase in temperature at the electrode- semiconductor interface was induced during laser annealing, reaching up to $1513{ }^{\circ} \mathrm{C}$ for $110 \mathrm{~mJ} \mathrm{~cm}{ }^{-2} \mathrm{XeCl}$ LA (Figure 14b). As suggested, the difference in thermal conductivities $(k)$ and diffusivities between Pt, Mo, and IGZO induces rapid heat conduction through the metal electrodes to the IGZO layer, therefore, promoting sharp heat accumulation at the interface. The latter process strongly affects the IGZO-electrode interface, causing the observed degradation in the device electrical characteristics, mainly in the case of narrow channel devices $(5 \mu \mathrm{m})$. On the other hand, use of the smaller penetration depth $\mathrm{KrF}$ excimer laser $(248 \mathrm{~nm}$ compared to $\mathrm{XeCl} 308 \mathrm{~nm})$, led to successful annealing of the devices even when lower energy fluences were used, resulting to TFTs with mobility of $\left.13 \mathrm{~cm}^{2} \mathrm{~V}^{-1} \mathrm{~s}^{-1}(80 \mathrm{~mJ} \mathrm{~cm})^{-2}\right)$. The finding highlighted $\mathrm{KrF}$ excimer laser annealing as a promising processing step for use in the field of metal-oxide electronics.

As part of a follow-up work, Bermundo et al. ${ }^{[195]}$ extended their work and examined the effect of single pulse $\mathrm{KrF}$ excimer laser treatment on IGZO TFTs comprised by a combination of $\mathrm{Mo} / \mathrm{Pt}$ or $\mathrm{Mo} / \mathrm{Au} \mathrm{S} / \mathrm{D}$ electrodes and a hybrid passivation layer of methylsilsesquioxane and phenylsilsesquioxane. Secondary ion mass spectrometry (SIMS) measurements revealed the presence of increased concentration of $\mathrm{H}$ and $\mathrm{C}$ in the IGZO surface of the passivated devices which originated from the (a)
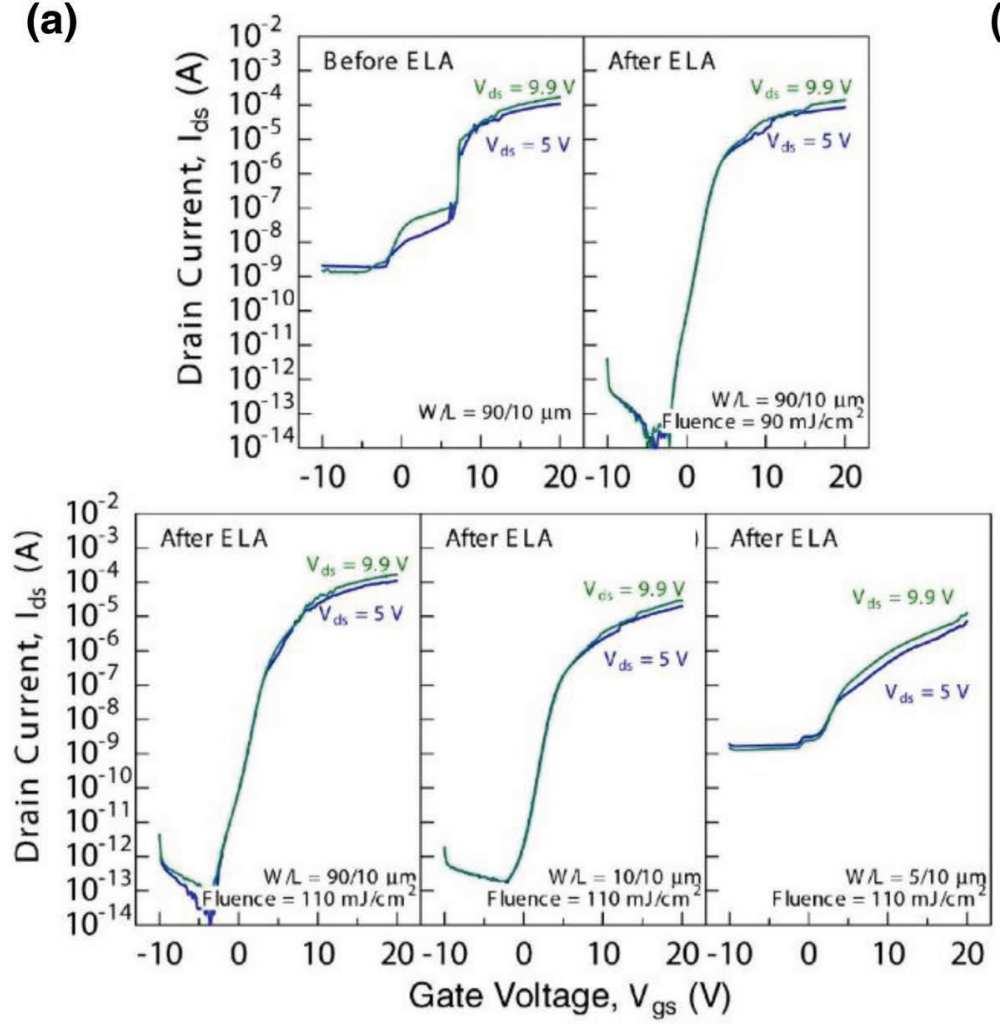

(b)
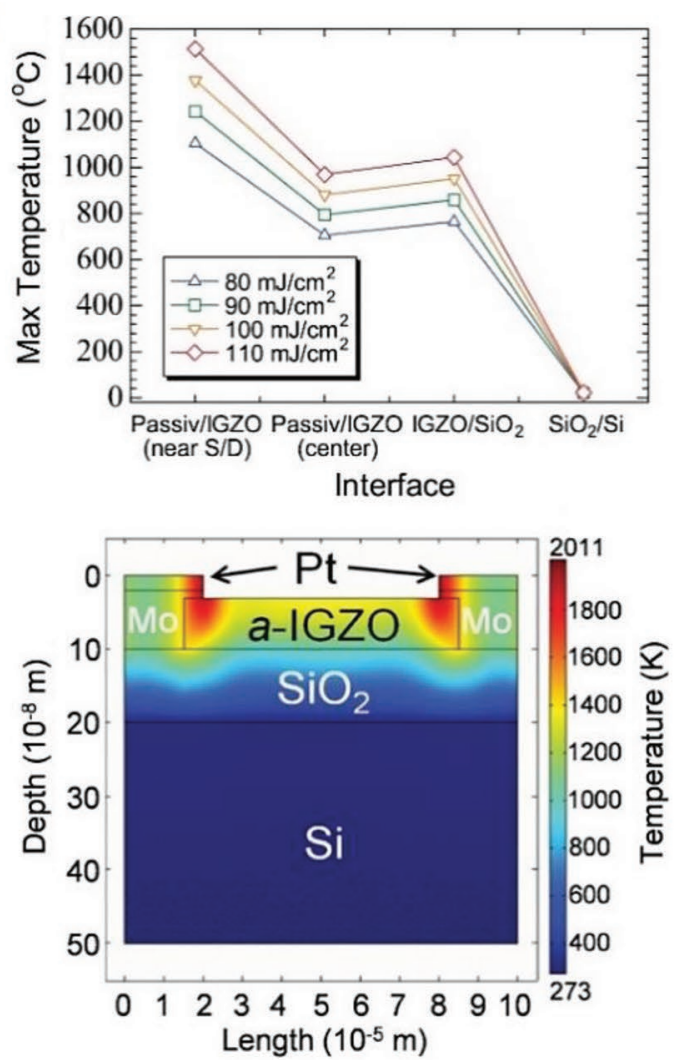

Figure 14. a) Transfer characteristics of a-IGZO TFTs passivated with PSQ, before and after LA with various laser fluences. b) 2D heat propagation simulation (COMSOL Multiphysics) of heat transfer into PSQ-passivated $\alpha$-IGZO TFTs during XeCl irradiation (top) and image of laser heating localization in the $\alpha$-IGZO TFT (bottom). Reproduced with permission. ${ }^{[194]}$ Copyright 2015, IOP Publishing. 
(a)

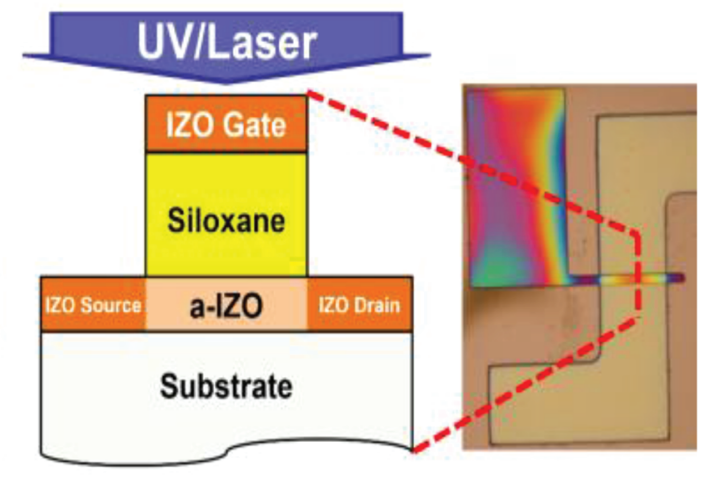

(b)
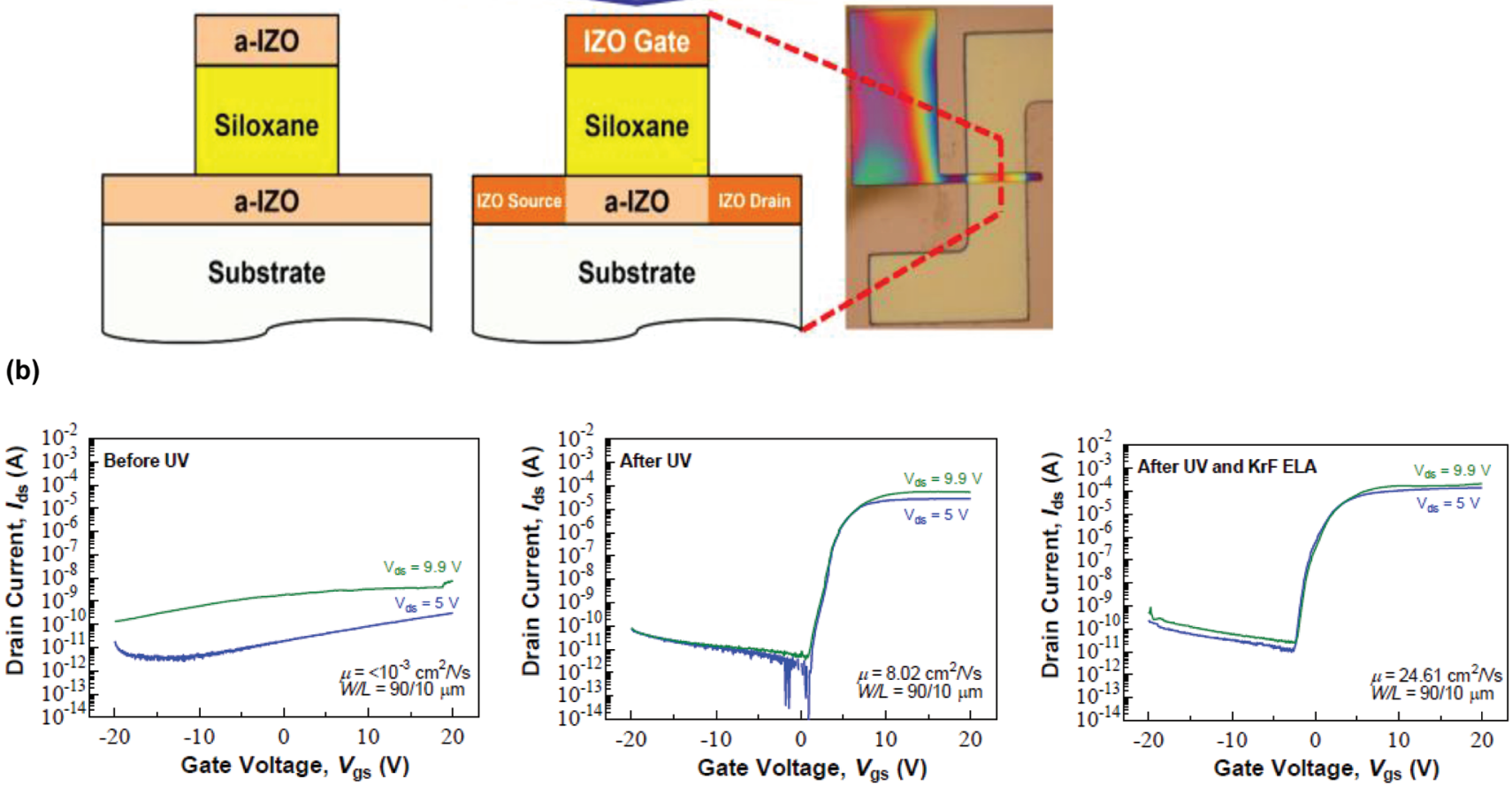

Figure 15. a) Schematic depiction of the manufacturing process steps of all-solution processed TFTs. b) Transfer characteristics of all-solution processed TFTs before UV exposure, after UV exposure and after UV exposure plus ELA treatment. Reproduced with permission. ${ }^{[96]}$ Copyright 2019 , The Society for Information Display.

thermal transfer phenomena of the molecules from the passivation layer during ELA. The examination of the XPS spectra in LA passivated, nonpassivated and furnace annealed passivated devices revealed the highest peak corresponding to the oxygen vacancies $\left(\mathrm{O}_{\mathrm{VAC}}\right.$ ratio $\left.=50.8 \%\right)$ in LA-treated devices, along with a higher concentration of $\mathrm{H} / \mathrm{C}$ related oxygen bonds in the IGZO surface, gradually decreasing with respect to the IGZO depth. By considering the presence of $\mathrm{H}$ atoms deeper in the surface, their role as electron donors (as they form $\mathrm{OH}$ bonds in IGZO and donate electrons when ionized) might increase the carrier mobility of the TFT slightly through passivation of shallow electron traps. In addition, the remarkable reported difference in the saturation mobility values between $\mathrm{Mo} / \mathrm{Pt}$ and $\mathrm{Mo} / \mathrm{Au}$-based IGZO TFTs (13 and $28.4 \mathrm{~cm}^{2} \mathrm{~V}^{-1} \mathrm{~s}^{-1}$, respectively), followed by variations in $\mathrm{V}_{\mathrm{ON}}$ values for the different electrode stacks, led to a further investigation of the diffusion of $\mathrm{Mo}, \mathrm{Au}$, and Pt atoms into the IGZO layer. Based on thermal diffusion simulations during LA, the absolute temperatures during irradiation can reach values up to $1100{ }^{\circ} \mathrm{C}$. Taking into consideration the melting points of $\mathrm{Mo}$, $\mathrm{Pt}$, and $\mathrm{Au}$ (2600, 1750, and $1050^{\circ} \mathrm{C}$, respectively), diffusion of $\mathrm{Au}$ into Mo and IGZO was considered during LA treatment and confirmed by energy-dispersive X-ray spectroscopy (EDS) measurements. This metal migration process was argued to be responsible for the increased electron density in the channel due to doping. This carrier concentration enhancement subsequently led to a mobility increase as well as shifting in the $V_{\mathrm{ON}}$ voltages to more negative values.
Finally, their most recent work focused on the fabrication of all-solution processed IZO TFT devices by exploiting photonic processing (UV lamp and laser treatment) in order to convert IZO into the conducting gate, source and drain electrodes. ${ }^{[196]}$ Siloxane was used as a gate dielectric, which was spin coated after the a-IZO channel layer. The second layer of a-IZO was then spin coated followed by an etching step that led to the formation of the final top-gate, bottom-contact TFT structure (Figure 15a). In order to transform the top IZO film along with the bottom exposed IZO areas into gate, source and drain electrodes, respectively, a UV lamp treatment $\left(115^{\circ} \mathrm{C}\right)$ step along with a single pulse of $\mathrm{KrF}$ excimer laser $\left(120 \mathrm{~mJ} \mathrm{~cm}{ }^{-2}\right)$ were utilized. Considering the absorption coefficients of the a-IZO in UV lamp and the KrF excimer laser irradiation, the penetration depths were calculated to be $75 \mathrm{~nm}$ (for UV lamp: $355 \mathrm{~nm}$ ) and $57 \mathrm{~nm}$ (for KrF: $248 \mathrm{~nm}$ ). Indeed, the successful conversion of all exposed a-IZO layers to their highly conductive state, with the exception of the channel layer, was subsequently confirmed. The transfer characteristics of the resulting TFTs (Figure 15b) indicated a remarkable enhancement in the channel ON current, the shift of $V_{\mathrm{TH}}$ close to $0 \mathrm{~V}$, and a remarkable electron mobility increase from $10^{-3} \mathrm{~cm}^{2} \mathrm{~V}^{-1} \mathrm{~s}^{-1}$, measured prior to optical posttreatment, to over $24 \mathrm{~cm}^{2} \mathrm{~V}^{-1} \mathrm{~s}^{-1}$ following UV/laser treatment.

In summary, pulsed laser annealing has already led to intriguing development in the area of metal-oxide electronics. The variety of annealing parameters offers the potential for a remarkable control of the thermally induced phenomena during laser irradiation, thus enabling stark enhancement in 
Table 3. Summary of reports on the application of pulsed laser annealing in metal-oxide TFT fabrication.

\begin{tabular}{|c|c|c|c|c|c|c|c|c|c|c|c|}
\hline Material & $\begin{array}{l}\text { Deposition } \\
\text { Method }\end{array}$ & Substrate & $\begin{array}{l}\text { Energy Density/ } \\
\text { Fluence } \\
{\left[\mathrm{m}^{\mathrm{cm}} \mathrm{cm}^{-2}\right]}\end{array}$ & $\begin{array}{c}\text { Laser } \\
\text { wavelength [nm] }\end{array}$ & $\mu\left[\mathrm{cm}^{2} \mathrm{~V}^{-1} \mathrm{~s}^{-1}\right]$ & $I_{\text {ON }} / I_{\text {OFF }}$ ratio & $V_{\mathrm{TH}}[\mathrm{V}]$ & $\mathrm{SS}\left[\mathrm{V} \mathrm{\operatorname {dec } ^ { - 1 } ]}\right.$ & Dielectric & Refs. & Year \\
\hline IGZO & $\begin{array}{l}\text { Magnetron } \\
\text { sputtering }\end{array}$ & Si wafer & $60-160$ & $308(\mathrm{XeCl})$ & 14 & NG & NG & NG & $\mathrm{SiO}_{2}$ & [183] & 2009 \\
\hline IGZO & $\begin{array}{l}\text { Magnetron } \\
\text { sputtering }\end{array}$ & Polymer & 60-190 & $308(\mathrm{XeCl})$ & 15.6 & NG & 0.5 & 0.13 & $\mathrm{SiO}_{2}$ & [185] & 2009 \\
\hline IGZO & $\begin{array}{l}\text { Magnetron } \\
\text { sputtering }\end{array}$ & Glass & $90-150$ & $308(\mathrm{XeCl})$ & 14 & NG & NG & NG & $\mathrm{SiO}_{2}$ & [184] & 2009 \\
\hline IGZO & RF sputtering & $\mathrm{Si}$ & $10-25$ & 266 (Nd: YAG) & 8 & $>10^{7}$ & 0 & 0.3 & $\mathrm{SiN}_{x}$ & [77] & 2010 \\
\hline IGZO & APPJ & $\mathrm{Si}$ & $0-250$ & $248(\mathrm{KrF})$ & 21.2 & $7 \times 10^{5}$ & NG & 0.48 & $\mathrm{ZrO}_{2}$ & [187] & 2014 \\
\hline IGZO & AP-PE-CVD & $\mathrm{Si}$ & 50-200 & $248(\mathrm{KrF})$ & 10 & $4.7 \times 10^{7}$ & 0.3 & 0.15 & $\mathrm{LaAl}_{2} \mathrm{O}_{3} / \mathrm{ZrO}_{2}$ & [188] & 2018 \\
\hline $\mathrm{ZnO}(\mathrm{NW})$ & VLS/drop & Sapphire & 50-100 & $248(\mathrm{KrF})$ & 27.9 & $3 \times 10^{2}$ & NG & 5.39 & $\mathrm{SiO}_{2}$ & [190] & 2009 \\
\hline $\mathrm{ZnO}$ & RF sputtering & $\mathrm{Si}$ & 100 & $248(\mathrm{KrF})$ & 5.08 & 8.49 & 0.6 & NG & $\mathrm{SiO}_{2}$ & [189] & 2010 \\
\hline $\mathrm{ZnO}$ & PE CVD & Glass & $0-300$ & $248(\mathrm{KrF})$ & 19.27 & $>10^{5}$ & 1.1 & NG & $\mathrm{SiO}_{2}$ & [191] & 2019 \\
\hline IGZO & RF sputtering & Glass & 300 & $308(\mathrm{XeCl})$ & 9.4 & NG & -0.8 & 0.22 & $\mathrm{SiO}_{2}$ & [193] & 2017 \\
\hline ZnO NPs & Drop casting & $\mathrm{Si}$ & $100-160$ & $248(\mathrm{KrF})$ & 0.106 & $>10^{4}$ & 5 & NG & $\mathrm{SiO}_{2}$ & [118] & 2009 \\
\hline IGZO & Spin coating & Glass & $0-740$ & 355 (Nd:YAG) & 7.65 & $2.8 \times 10^{7}$ & NG & NG & $\mathrm{SiO}_{2}$ & [186] & 2010 \\
\hline $\ln _{2} \mathrm{O}_{3}$ & Spin coating & $\mathrm{Si}$ & $300-450$ & $248(\mathrm{KrF})$ & $6-10$ & $>10^{5}$ & $\approx 5$ & NG & $\mathrm{SiO}_{2}$ & [99] & 2017 \\
\hline ICZO & RF sputtering & $\mathrm{Si}$ & (90-110(80-100) & $\begin{array}{l}308 \mathrm{XeCl} \\
(248 \mathrm{KrF})\end{array}$ & $12.5(13)$ & $10 \times 10^{10}$ & $3(0.5)$ & NG & $\mathrm{SiO}_{2}$ & [194] & 2015 \\
\hline IGZO & RF sputtering & $\mathrm{Si}$ & $75-80$ & $248(\mathrm{KrF})$ & 28.4 & NG & $-5.5\left(\mathrm{~V}_{\mathrm{ON}}\right)$ & NG & $\mathrm{SiO}_{2}$ & [195] & 2017 \\
\hline IZO & Spin coating & $\mathrm{Si} / \mathrm{SiO}_{2}$ & 120 & $\begin{array}{c}248 \text { (KrF) } \\
(+ \text { UV) }\end{array}$ & 24.61 & NG & 0.45 & 0.29 & Siloxane & [196] & 2019 \\
\hline IZO & Spin coating & Glass & 300 & $248(\mathrm{KrF})$ & 0.58 & $>10^{5}$ & 2.84 & NG & $\mathrm{SN}_{x}: \mathrm{H} / \mathrm{SiO}_{x}$ & [113] & 2015 \\
\hline ZTO & Spin coating & $\mathrm{Si}$ & $0-390$ & $308(\mathrm{XeCl})$ & 0.95 & NG & 4.78 & NG & $\mathrm{SiO}_{2}$ & [197] & 2012 \\
\hline IZO & RF sputtering & $\mathrm{Si}$ & $30 / 97.5$ & $308(\mathrm{XeCl})$ & 37.7 & $>10^{7}$ & NG & 0.18 & $\mathrm{SiO}_{2}$ & [198] & 2013 \\
\hline
\end{tabular}

the optoelectronic properties of metal-oxide layers and their devices. Table 3 summarizes most of the key developments to date involving the use of laser annealing for the optimization and/or manufacturing of metal-oxide electronics.

\section{Flash Lamp Annealing}

Similar to DUV annealing and LA, flash lamp annealing allows for facile thermal treatment of thin layers of different materials on large area substrates through the absorption of a highly energetic light pulse on the time scale of $10^{-6}$ to $10^{-3} \mathrm{~s}$. The absorbed optical energy is then transformed into thermal energy at the near-surface region of the illuminated layer(s). Due to the formation of a vertical thermal gradient, the temperature in the deeper regions of the layer is significantly lower, which reduces the thermal load in the bulk of the material. Furthermore, thermal conduction into the bulk of the substrate facilitates the rapid cooling of the near-surface region after a short-time exposure. In this way, the thermal equilibrium is reached within several milliseconds preventing damage to all involved materials, making FLA an attractive technology for the rapid high-temperature treatment of metal oxides even when deposited onto thermally sensitive substrate materials.

\subsection{A Brief History of Flash Lamp Annealing}

After the invention of the very first electric flash lamp in the late 1930s for professional photography, ${ }^{[199]}$ it took almost forty years until its potential in semiconductor processing was demonstrated. In their pioneering work, Kachurin and Nidaev used a piece of ion-implanted silicon which was accidentally exposed to a xenon laser pump. ${ }^{[200]}$ Following laser exposure, it was observed that the sample exhibited improved electrical properties, providing evidence that intense xenon light pulses can be used to heal lattice defects induced during ion-implantation. Since then FLA has been extensively used in the conventional semiconductor industry for the annealing of ion-implanted semiconductors, ${ }^{[201-203]}$ crystallization of amorphous thin films ${ }^{[204-206]}$ or the recrystallization of heteroepitaxial layers. ${ }^{[207,208]}$

During the last two decades, FLA has become a promising technology for printed electronics allowing the high-temperature sintering of metal nanoparticle inks for the realization of conductive patterns on arbitrary substrates. ${ }^{[209,210]}$ Applications include the fabrication of current collecting grids for solar cells, ${ }^{[211]}$ metal contacts for organic TFTs, ${ }^{[212]}$ electrodes for multilayer ceramic capacitors, ${ }^{[213]}$ and piezoelectric materials. ${ }^{[214,215]}$ Also, owing to the rapid processing times, the integration of FLA into a roll-to-roll manufacturing line was successfully 
demonstrated for the mass production of polymer solar cell modules $^{[216]}$ and radio frequency identification (RFID) tags ${ }^{[217]}$ on PET substrates. Finally, FLA has recently gained significant interest in the photonic treatment of metal-oxide semiconductors and dielectrics derived from solution or high-vacuum processes precursors/materials for application in various types of electronics.

\subsection{Flash Lamp Annealing System}

The components of a primary flash lamp system include one or more individual flash lamps, a reflector to direct the light toward the sample, and a quartz window to separate the sample from the flash lamp. A flash lamp comprises an anode and a cathode, which are hermetically sealed in a quartz body filled with a specific noble gas (Xe, $\mathrm{Kr}$, or $\mathrm{Ar}$ ). The emission spectrum is typically very broad ranging from the UV to the IR region. Even though all noble gases create a similar spectrum, dominant emission regions can be different from one to the other (Figure 16). ${ }^{[218]}$ For the operation of a typical flash lamp two different circuits are of significant importance: i) a pulse forming network and ii) a trigger circuit. Depending on the pulse forming network and the shape of the optical pulse, the surface temperature of the irradiated sample can be controlled. [219]

Furthermore, by using suitable optical filters, specific sections of the emission spectrum can be either blocked or selectively allowed to pass. The UV region, for example, can be utilized for photochemical conversions of solution-processed metal-oxide precursors. ${ }^{[50]}$ To this end, the characteristic short penetration-depth of the UV light can be advantageous, especially for thin-film fabrication processes on temperature sensitive substrates. ${ }^{[220]}$

In the FLA process, four parameters are playing key roles, namely, the energy density which is derived from the integral intensity of the flash pulse, the pulse length calculated by the full width at half maximum of the pulse, the number of pulses and lastly the fire rate defined as the number of pulses per second. Depending on the targeted materials, precise adjustments of these parameters allows for optimal annealing

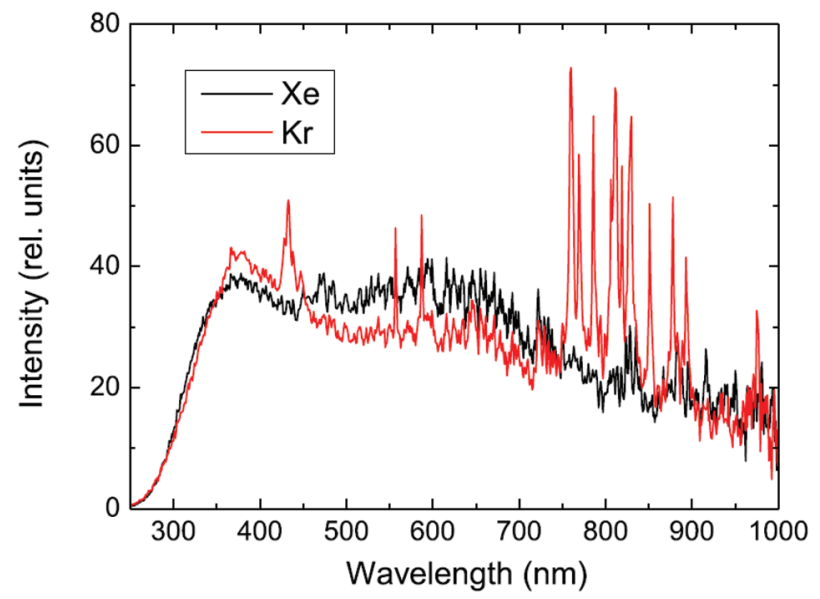

Figure 16. The light emission spectra of $\mathrm{Xe}$ and $\mathrm{Kr}$ flash lamps. Reproduced with permission. ${ }^{[218]}$ Copyright 2016, IOP Publishing. conditions. Rigid substrate materials such as silicon or glass can withstand high temperatures owing to their glass transition temperature $\left(T_{\mathrm{g}}\right)$. This makes it possible to treat thin films atop with high energy densities, fast fire rates and an increased number of pulses. ${ }^{[221]}$ On the other hand, temperature sensitive substrates such as flexible polymers or metal foils cannot be processed with the same FLA parameters because of their sensitivity to extreme light, decomposition or melting. ${ }^{[221]}$ To minimize those effects, shorter pulse lengths and lower fire rates with a higher number of pulses are often employed to obtain optimal results.

\subsection{Temperature Formation and Process Constraints}

Obtaining high temperatures in a short period of time with FLA strongly depends on the sample and its light absorption characteristics. Under flashlight irradiation, the surface temperature of the absorptive element (metal films, MO precursors, metal nanoparticles, etc.) can reach up to $\approx 1000{ }^{\circ} \mathrm{C}$. ${ }^{[21]}$ Since the conventional temperature measurement devices, such as thermocouples, rely on physical contact with the sample, measurement of temperature under FLA represent a significant challenge due to the short timescales involved during flashing. In order to eliminate this issue, optical-based measurement techniques have been suggested. ${ }^{[222-224]}$ Alternatively, simulation-based techniques can also be utilized to estimate the temperature range on the sample surface. ${ }^{[29,218]}$

During FLA, the amount of absorbed light is governed by the absorption coefficient, the thickness of the targeted film and the reflection from the surface of the sample. According to the Beer-Lambert law, the total absorption is directly proportional to the absorptivity $(\alpha)$ and thickness $(x)$ of the materials. Across the defined spectral range with the illuminated area $A$, the approximate heat $(Q)$ under incident radiation $I_{0}(\lambda)$ can be estimated as ${ }^{[225]}$

$Q=A \iint I_{0}(\lambda) e^{-\alpha x} \mathrm{~d} \lambda \mathrm{d} x$

According to Equation (2), the temperature within the film can be calculated using

$\rho C(T) \frac{\partial T}{\partial t}=\nabla(\kappa \nabla \mathrm{T})+\mathrm{Q}$

where $\rho$ is the density, $C$ is the heat capacity, and $\kappa$ is the thermal conductivity of the absorptive element (i.e., the sample). The thermal diffusivity $(D)$ and the thermal diffusion length (l) can then be calculated using

$D=\frac{\kappa}{\rho C}$
$l=2 \sqrt{\frac{\kappa \tau}{\rho C}}$

where $\tau$ is the thermal equilibrium time. It is found that substrates such as polymers have shorter thermal diffusion lengths as well as diffusion-length-to-thickness-ratio compared 
to absorbing thin films. Even though high temperatures can be reached on the surface of the absorptive thin films in very short times, the substrate material experiences significantly lower temperatures. The thermal equilibrium times for both the substrate and thin film $\left(\tau_{\text {substrate }}\right.$ and $\left.\tau_{\text {film }}\right)$ can be calculated separately using ${ }^{[221]}$

$\tau_{i}=\frac{C_{i} \rho_{i} l_{i}^{2}}{4 \kappa_{i}}$

In order to reach a high temperature within the absorber layer, while maintaining the substrate temperature low, the pulse duration ( $\left.t_{\text {pulse }}\right)$ has to be adjusted in a way that it is longer than $\tau_{\text {film }}$ but shorter than $\tau_{\text {substrate }}$. As soon as the light pulse is off, the high temperature on the thin film is dissipated by conducting the heat to the substrate, by thermal radiation or by heat convection. Thus, the thickness of the substrate material should be much thicker than the thin film to resist the thermal load. It is important to note, that if not adjusted precisely, temperatures generated throughout the thin film can damage the polymer-based substrate. In brief, the overall conditions are given in Figure 17.

Despite the many advantages of FLA over other annealing methods, still, several constraints should be taken into account to find the optimum process conditions. During FLA, the formation of stress and strain between the thin film and the carrier substrate materials are taking place because of the different thermal expansions of the film and the substrate. If the stress exceeds a specific magnitude (i.e., yield stress), the delamination and wrinkling of the film can deteriorate the function of the device. ${ }^{[226]}$ This scenario is especially seen in the flexible

(a)

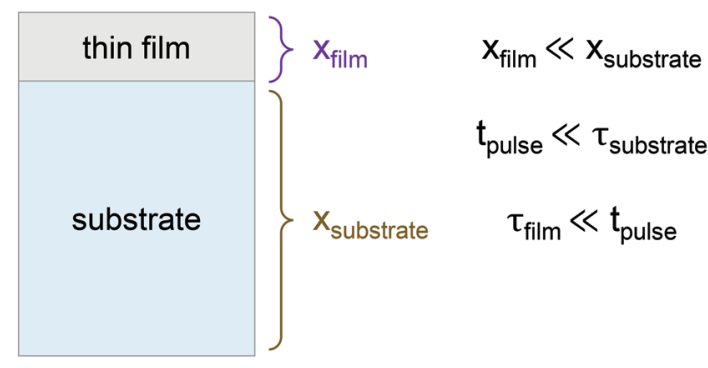

(b)

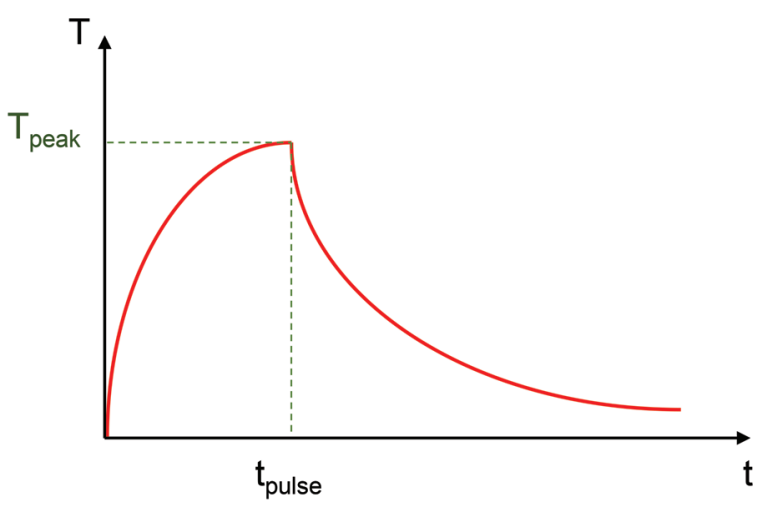

Figure 17. a) Schematic cross section of the substrate and thin film. b) Temperature-time relation under flashlight annealing. carrier substrates with high thermal expansion coefficients. However, this problem can be overcome with the addition of a buffer layer between the film and the substrates, which has a thermal expansion coefficient between that of the film and the substrate. ${ }^{[227]}$ Additionally, mild heating of the substrate while processing can be a solution to reduce the thermal gradients and the stress level.

Besides the mechanical issues induced by the temperature gradient, the formation of gaseous products can trigger delamination, microcracks and microstructural changes during and after flashing. This problem is especially relevant for thin films which are fabricated via solution-based techniques employing precursors. ${ }^{[228]}$ To overcome this, a mild drying step on a hot plate and/or a less intense FLA can be applied. ${ }^{[22]}$ In addition to the solvent, volatile compounds and organic residuals leave the film by forming gas bubbles and inducing microcracks. In a study conducted with PE-CVD process, microstructural changes were observed after the flashing of a SiON layer, owing to the rapid removal of hydrogen from the layer as a gas. ${ }^{[230]}$

An additional critical parameter is the homogeneity of the light emitted from the flash lamp over the irradiated area so that the temperature distribution throughout the surface of the irradiated sample remains as homogeneous as possible. Any fluctuation in the temporal and spatial energy density can create a considerable temperature gradient across the layer resulting in the formation of inhomogeneous microstructures. Moreover, the quality of the irradiated samples itself affects heat formation and distribution. Local variations, such as roughness, absorption and reflectivity can generate nonhomogeneous heating of the substrate. These conditions are critical in achieving consistent and reproducible results via FLA.

In addition to these limitations, use of FLA for the conversion of soluble metal-oxide precursors on fully transparent substrates is a challenging endeavor. Considering the fact that the majority of sol-gel metal-oxide precursors do not absorb much light over the relevant part of the emitted spectrum of a flash lamp, a light absorbing element that acts as a heating element is required to convert the precursor to the final metal oxide. ${ }^{[231]}$ However, for all-oxide devices and circuits, the utilization of such elements is very challenging. To address this, absorptive precursor molecules are often added to the precursor solution to assist with the decomposition process during flashing. ${ }^{[232]} \mathrm{A}$ similar approach has been used with the continuous DUV conversion process. ${ }^{[50,51]}$

\subsection{FLA Processing of Metal-Oxide Thin-Film Transistors}

Following its application in silicon electronics, FLA started to attract attention for the growth of various metal-oxide layers including dielectrics, semiconductors and transparent conductive electrodes. ${ }^{[233-235]}$ The use of FLA for the fabrication of metal-oxide TFTs falls into different categories depending on the particular fabrication method employed. Table 4 summarizes results on metal-oxide TFTs subjected to FLA using different processing parameters and deposition methods.

As in the case of laser and DUV methods, FLA can be used as a postdeposition step following the deposition of the metal oxide. Even though the as-processed metal-oxide layers 
Table 4. Summary of reports that use FLA for the fabrication of metal-oxide TFTs.

\begin{tabular}{|c|c|c|c|c|c|c|c|c|c|c|}
\hline Material & $\begin{array}{c}\text { Deposition } \\
\text { Method }\end{array}$ & Substrate & $\begin{array}{l}\text { Energy, energy } \\
\text { density, power }\end{array}$ & $\mu\left[\mathrm{cm}^{2} \mathrm{~V}^{-1} \mathrm{~s}^{-1}\right]$ & $I_{\mathrm{ON}} / I_{\mathrm{OFF}}$ Ratio & $V_{\mathrm{TH}}[\mathrm{V}]$ & $\mathrm{SS}\left[\mathrm{V} \mathrm{\textrm {dec } ^ { - 1 } ]}\right.$ & Dielectric & Ref. & Year \\
\hline IGZO & Spin coating & Si wafer & $100 \mathrm{~J} \mathrm{~cm}^{-2}$ & 2.67 & $10^{8}$ & -12 & 1.8 & $100 \mathrm{~nm} \mathrm{SiO} 2$ & [25] & 2014 \\
\hline IGZO & Sputtering & Si wafer & $3.95 \mathrm{~kW} \mathrm{~cm}{ }^{-2}$ & 7.8 & $10^{8}$ & 8.1 & 0.22 & $100 \mathrm{~nm} \mathrm{SiO} 2$ & [236] & 2015 \\
\hline IGZO & Sputtering & Si wafer & $8 \mathrm{~kW}$ & 10.62 & $8.46 \times 10^{4}$ & -0.11 & 0.56 & 300 nm SiO & [237] & 2014 \\
\hline $\mathrm{ZnO}$ & Spin coating & Si wafer & NG & 0.058 & $10^{6}$ & 16.21 & 0.55 & $150 \mathrm{~nm} \mathrm{SiO} 2$ & [228] & 2015 \\
\hline IGZO & Printing & Si wafer & $100 \mathrm{~J} \mathrm{~cm}^{-2}$ & 0.1 & $3.210^{5}$ & -15 & 2 & $300 \mathrm{~nm} \mathrm{SiO} 2$ & [238] & 2015 \\
\hline IZO & Spin coating & Si wafer & $250000 \mathrm{~J}$ & 2.4 & $2.4 \times 10^{6}$ & 6.72 & 1.65 & $100 \mathrm{~nm} \mathrm{SiN}$ & [239] & 2016 \\
\hline IGZO & Spin coating & Si wafer & $2000 \mathrm{~J}$ & 7 & NG & NG & NG & $100 \mathrm{~nm} \mathrm{SiO} 2$ & [240] & 2016 \\
\hline IGZO & Spin coating & Polyimide & $2000 \mathrm{~J}$ & 4.5 & $10^{9}$ & 4 & 1.2 & PS/PVP & [240] & 2016 \\
\hline $\ln _{2} \mathrm{O}_{3}$ & Spin coating & Si wafer & $207 \mathrm{~J}$ & 38.9 & $10^{4}$ & NG & NG & $100 \mathrm{~nm} \mathrm{SiO} 2$ & {$[224]$} & 2016 \\
\hline $\ln _{2} \mathrm{O}_{3}$ & Printing & PEN & $400 \mathrm{~J}$ & 8 & $2 \times 10^{6}$ & 0.08 & 0.12 & $\begin{array}{c}\text { solid polymer } \\
\text { electrolyte }\end{array}$ & [241] & 2017 \\
\hline $\mathrm{In}_{2} \mathrm{O}_{3} / \mathrm{ZnO}$ & Spin coating & Glass & $5 \mathrm{~J} \mathrm{~cm}^{-2}$ & 36 & $2 \times 10^{3}$ & 0.2 & NG & $\mathrm{Al}_{2} \mathrm{O}_{3} / \mathrm{ZrO}_{2}$ & [229] & 2017 \\
\hline IGZO & Spin coating & Si wafer & $207 \mathrm{~J}$ & 8.6 & $10^{4}-10^{5}$ & NG & 1.7 & $200 \mathrm{~nm} \mathrm{SiO} 2$ & [242] & 2018 \\
\hline $\mathrm{ZnON}$ & Sputtering & Si wafer & $40 \mathrm{~J} \mathrm{~cm}^{-2}$ & 48.4 & $\approx 10^{9}$ & 0.26 & 0.38 & $100 \mathrm{~nm} \mathrm{SiO} 2$ & [243] & 2019 \\
\hline IGZO & Spin coating & Si wafer & $130 \mathrm{~J} \mathrm{~cm}^{-2}$ & 7.7 & $3 \times 10^{6}$ & NG & NG & $300 \mathrm{~nm} \mathrm{SiO} 2$ & {$[244]$} & 2019 \\
\hline
\end{tabular}

(semiconductors, dielectrics, etc.) may already yield functional TFTs, an additional annealing step is sometimes necessary in order to improve their performance characteristics. ${ }^{[24]}$ This step is typically carried out for several reasons including activation of electronic dopants, activation or elimination of microstructural phases, the enhancement of material crystallinity, and the densification of metal-oxide layers. In other words, FLA can be used as a substitute for conventional thermal annealing but with the added advantage of significantly shorter process time.

For the use of FLA in solution-based processing methodologies, two different approaches are often adopted. The first approach is based on a pure thermal process in which the lamp's radiant energy is absorbed by a component in the device structure and converted to thermal energy. The high temperature generated within the absorbing element (metallic layer etc.) can then be utilized to initiate the thermochemical conversion of the metal-oxide precursor deposited onto or beneath the absorbing element. However, the presence of such light absorbing element hinders the fabrication of fully transparent devices. The second approach relies on the usage of the UV part of the flashlight spectrum to initiate a photochemical reaction in the metal-oxide precursor just like in the case of continuous DUV conversion process. However, the need for UV absorptive elements in the precursor formulation still plays an important role in the formation of the final metal oxide.

\subsubsection{FLA as a Postdeposition Process Step}

The first attempt at using FLA as a postdeposition technique for oxide materials was conducted in 1985 by Hensel et al. on oxygenimplanted $\mathrm{SiO}_{2}$ dielectric layers. ${ }^{[233]}$ XPS and IR spectroscopy analyses showed that $\mathrm{SiO}_{2}$ films processed with FLA at $1400{ }^{\circ} \mathrm{C}$ for $1 \mathrm{~ms}$ had similar properties to the sample annealed in a furnace for $2 \mathrm{~h}$ at $1000{ }^{\circ} \mathrm{C}$. Following, FLA was applied to hafnium oxide $\left(\mathrm{HfO}_{2}\right)$ dielectric layers deposited by ALD at $275{ }^{\circ} \mathrm{C}$ after a short preheating step at $400{ }^{\circ} \mathrm{C} .{ }^{[245]}$ The results revealed a similar crystallization of the films annealed with FLA at $20 \mathrm{~J} \mathrm{~cm}^{-2}$ compared to films subjected to a $600{ }^{\circ} \mathrm{C}$ rapid thermal annealing process. The FLA treated $\mathrm{HfO}_{2}$ layers showed higher capacitance values than samples exposed to an oxygen plasma assisted annealing but similar leakage current densities. Lehmann and Borany reported the growth of $\mathrm{LaLuO}_{3}$ and $\mathrm{LaScO}_{3}$ dielectric films via FLA. ${ }^{[246,247]}$ Similar to previous studies, a preheating annealing step $\left(570^{\circ} \mathrm{C}\right.$ for $\left.40 \mathrm{~s}\right)$ was applied before FLA in order to decrease the stress formation in the wafer. The temperatures reached during FLA were estimated to be between $1000-1200{ }^{\circ} \mathrm{C}$ with a pulse length of $3 \mathrm{~ms}$. The formation of nanocrystals within the amorphous structure was observed which depended on the maximum temperature reached.

The application of FLA on sputtered IGZO layers deposited on $\mathrm{Si} / \mathrm{SiO}_{2}$ substrates was first proposed by Noh et al. (Figure 18a). ${ }^{[236]}$ As compared to TFTs prepared by conventional TA at $250{ }^{\circ} \mathrm{C}$ for $1 \mathrm{~h}$ in air $\left(\mu=6.7 \mathrm{~cm}^{2} \mathrm{Vs}^{-1}\right)$, FLA treated TFTs exhibited higher electron mobility of $7.8 \mathrm{~cm}^{2} \mathrm{~V}^{-1} \mathrm{~s}^{-1}$. Besides, the $V_{\mathrm{TH}}$ of the FLA devices showed smaller shifts and better stability under positive bias stress. These results highlighted the potential of FLA as a promising and rapid method for improving the performance of metal-oxide TFTs.

The effect of FLA on the illumination stability of IGZO TFTs was first reported by Jeong et al. ${ }^{[237]}$ The IGZO layers were deposited by RF-sputtering and annealed at $450{ }^{\circ} \mathrm{C}$ followed by FLA. The results indicated an increase of the electron mobility with increasing irradiation power and number of pulses reaching a maximum value of $10.62 \mathrm{~cm}^{2} \mathrm{~V}^{-1} \mathrm{~s}^{-1}$; a significantly higher value as compared to that obtained for thermally annealed TFTs $\left(0.2 \mathrm{~cm}^{2} \mathrm{Vs}^{-1}\right)$. Moreover, the FLA treated transistors showed improved electrical stability under white light illumination manifested in significantly lower changes in $\mu$ and $V_{\mathrm{TH}}$.

Recently, Jeong et al. ${ }^{[243]}$ reported the effect of visible light FLA on ZnON TFTs. The ZnON film was deposited on $\mathrm{Si} / \mathrm{SiO}_{2}$ 

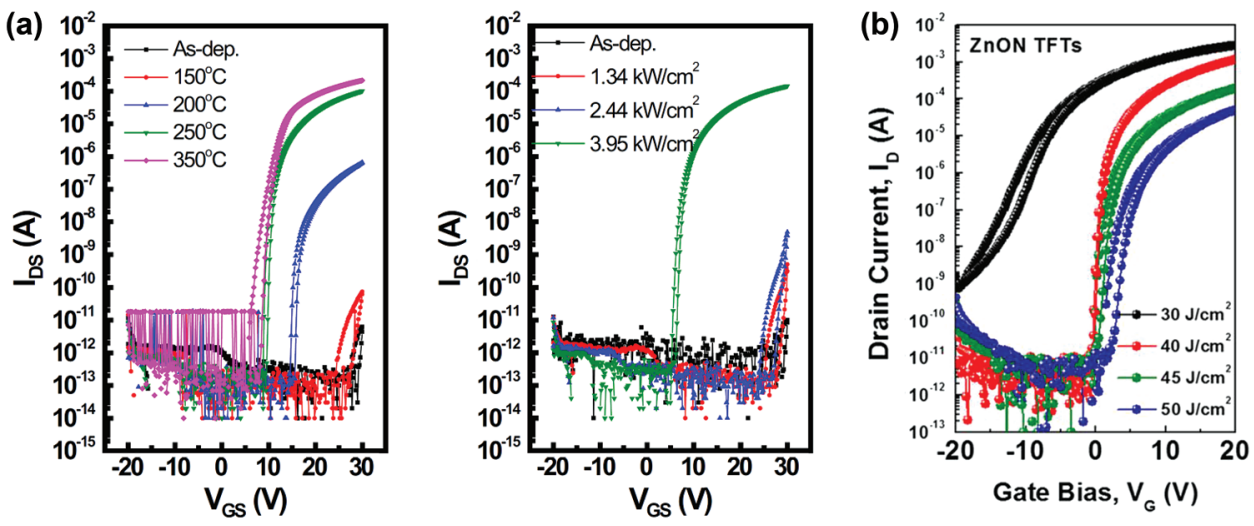

Figure 18. a) Transfer characteristics of thermal and FLA treated TFTs subjected to different annealing temperatures and flashing conditions. Reproduced with permission. ${ }^{[236]}$ Copyright 2015, IEEE. b) Transfer characteristic of ZnON TFTs subjected to different flashing energies. Reproduced with permission. ${ }^{[243]}$ Copyright 2019, American Chemical Society.

wafers by reactive sputtering of $\mathrm{Zn}$ in a mixed atmosphere of nitrogen and oxygen. The fabricated films were annealed using a flash lamp that emits in the visible spectrum at a pulse length of $20 \mathrm{~ms}$ and radiant energy densities ranging from $30-50 \mathrm{~J} \mathrm{~cm}^{-2}$. The investigations revealed that as-deposited films and films post-treated with flashes at low energies $\left(30 \mathrm{~J} \mathrm{~cm}^{-2}\right.$ ) remained too conductive preventing low off currents in the transistor channel due to the possible formation of conductive stoichiometric $\mathrm{Zn}_{3} \mathrm{~N}_{2}$ phases and oxygen vacancy defects in the layer. However, by increasing the pulse energies, the total carrier concentration inside the channel region was gradually decreased, leading to a reduction of the off current levels of the transistor (Figure 18b). At flashing energy of $40 \mathrm{~J} \mathrm{~cm}^{-2}$ the maximum mobility value of $48.4 \mathrm{~cm}^{2} \mathrm{~V}^{-1} \mathrm{~s}^{-1}$ was reached with suppressed hysteresis. However, for higher energy values $\left(>40 \mathrm{~J} \mathrm{~cm}^{-2}\right)$, ZnON TFT performance decreased due to the formation of $\mathrm{ZnO}$ and nonstoichiometric $\mathrm{Zn}_{x} \mathrm{~N}_{y}$ at the $\mathrm{ZnON} / \mathrm{SiO}_{2}$ interface. Although only a slight change in the $V_{\mathrm{TH}}$ value under positive bias stress was observed, the authors found a more pronounced change under negative bias stress. This stability issue in the negative region was attributed to the nonstoichiometric $\mathrm{Zn}_{x} \mathrm{~N}_{Y}$ in the $\mathrm{ZnON}$ film and the changes in nitrogen bonding states due to the hole trapping property of $\mathrm{N}-\mathrm{N}$ bonds.

\subsubsection{Photonic Conversion of Solution-Processed Metal-Oxide Transistors}

The primary effects of FLA on solution processed metal oxides include: to evaporate the solvent, hydrolyze the precursor, promote layer condensation and densification, and ultimately to crystallize the formed metal oxide. Different approaches can be taken for FLA processing depending on the device structure, chemical composition of the precursor formulation, and/or the method of coating. For instance, the gate metal (or any other light absorbing material) in a bottom-gate TFT configuration, can be used to absorb part of the radiant energy over a broad spectrum and convert this into thermal energy. This thermal energy can then be utilized for the chemical conversion of the solution-deposited precursor layer. Effectively, the gate electrode acts as a microhotplate. ${ }^{[229]}$ On the other hand, in the absence of any such absorbing component, absorptive molecules can be added into the formulation and assist with the direct photo conversion as in the case of the DUV process. ${ }^{[232]}$

Tetzner et al., were the first to use FLA to treat a sol-gel $\mathrm{HfO}_{2}$ precursor layer deposited on Ti/Pt coated glass wafers. ${ }^{[231]}$ Hafnium isopropoxide-based sol-gel solution was spun-cast on $\mathrm{Ti} / \mathrm{Pt}$ coated glass wafers and dried at $150{ }^{\circ} \mathrm{C}$ for $5 \mathrm{~min}$ before FLA processing. Rather than applying a single pulse during the FLA process on the sol-gel layers, pulses shaped into three regimes were used for the conversion as seen in Figure 19a. A peak temperature of $\approx 1200{ }^{\circ} \mathrm{C}$ on the wafer surface was calculated after the exposure at the radiant energy density of $6 \mathrm{~J} \mathrm{~cm}^{-2}$ using computer simulations. Moreover, a significant color change of the sol-gel layers was observed after three shaped pulses due to the conversion and densification of the thin film (Figure 19b). When sol-gel derived $\mathrm{HfO}_{2}$ layers were used as the dielectric layer in a capacitor, the authors found a reduction of the leakage current densities by three orders of magnitude upon FLA with the devices yielding a high areal capacitance value of $0.4 \mu \mathrm{F} \mathrm{cm}{ }^{-2}$ (Figure 19c). The flash treated $\mathrm{HfO}_{2}$ layer was then successfully incorporated into organic low operating voltage TFTs.

The FLA treatment of a solution-based IGZO metal-oxide semiconductor used for the active channel in a TFT was reported by Yoo et al. ${ }^{[25]}$ In this work, the authors deposited an IGZO precursor on $\mathrm{Si} / \mathrm{SiO}_{2}$ substrates via spin-coating and compared the performance of FLA treated devices exposed to 10 flash pulses with radiant energies of $100 \mathrm{~J} \mathrm{~cm}^{-2}$ with samples that were thermally annealed at $500{ }^{\circ} \mathrm{C}$ for $1 \mathrm{~h}$. FLA induced the formation of metal-oxygen networks as verified by Fourier-transform infrared spectroscopy (FTIR). Due to the high optical transparency of the IGZO precursor solution, the authors assumed a significant heat generation at the interface with the $\mathrm{Si}$ substrate resulting in the thermochemical conversion of the precursor. The FLA treated IGZO TFTs showed an on/off current ratio of $10^{8}, \mathrm{SS}=1.8 \mathrm{~V} \mathrm{dec}^{-1}$ and electron mobility of $2.67 \mathrm{~cm}^{2} \mathrm{~V}^{-1} \mathrm{~s}^{-1}$. The latter value was ten times higher than TFTs prepared via thermal annealing $\left(0.3 \mathrm{~cm}^{2} \mathrm{~V}^{-1} \mathrm{~s}^{-1}\right)$ (Figure 20a,b). 

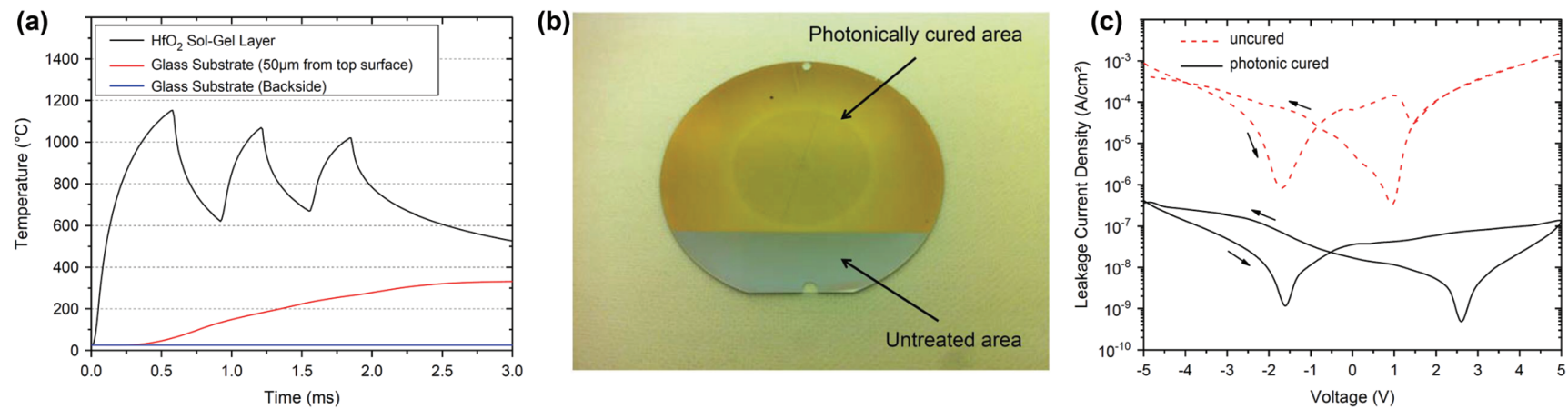

Figure 19. a) Simulated evolution of temperature with time upon FLA for the $\mathrm{HfO}_{2}$ surface, the glass top surface and the backside of the glass substrates. b) Image of a partly exposed glass/Ti/Pt/precursor wafer to FLA. Stark differences between the treated and untreated areas of the wafers can be seen. c) Leakage current densities of devices based on nonflashed and flashed $\mathrm{HfO}_{2}$ layers. Reproduced with permission. ${ }^{[231]}$ Copyright 2014, Elsevier.

Kang et al. used a surface thermometer and a 1D heat transfer model in order to estimate the temperatures during the FLA treatment. ${ }^{[224]}$ According to their simulation, the surface of the $\mathrm{Si} / \mathrm{SiO}_{2}$ substrate reached temperatures above $350{ }^{\circ} \mathrm{C}$ after the flash lamp irradiation with flashes featuring radiant energies of $207 \mathrm{~J}$ per pulse at a total irradiation time of $120 \mathrm{~s}$ with a fire rate of $15 \mathrm{~Hz}$. In addition, spin-coated $\mathrm{In}_{2} \mathrm{O}_{3}$ precursor layers on $\mathrm{Si} / \mathrm{SiO}_{2}$ substrate were flashed with the total irradiation times varying from 5 to $120 \mathrm{~s}$. For short irradiation times $(<5 \mathrm{~s})$, the devices did not show any transistor function. On the other hand, longer irradiation times yielded TFTs with electron mobilities reaching $2.42 \mathrm{~cm}^{2} \mathrm{~V}^{-1} \mathrm{~s}^{-1}$ for $10 \mathrm{~s}$, and $38.9 \mathrm{~cm}^{2} \mathrm{~V}^{-1} \mathrm{~s}^{-1}$ for $120 \mathrm{~s}$. However, the device fabricated using irradiation times of $120 \mathrm{~s}$ suffered from high off currents because of the increased oxygen vacancies that formed at high temperatures (Figure 21a). As a solution to this problem, a pulse of $10 \mathrm{~s}$ was repeated three times with breaks in between resulting in a reduced off current and optimum mobility of $10.3 \mathrm{~cm}^{2} \mathrm{~V}^{-1} \mathrm{~s}^{-1}$ as seen in (Figure $21 \mathrm{~b}$ ).

Kim et al. used a zinc ammonia hydroxide complex in order to fabricate zinc-oxide thin-film transistors on $\mathrm{Si} / \mathrm{SiO}_{2}$ substrates via FLA. ${ }^{[28]}$ The samples were exposed to a different number of pulses ranging from 9 to 45 while keeping the samples at a constant temperature of $90{ }^{\circ} \mathrm{C}$ in order to eliminate solvent residues. While the $\mathrm{ZnO}$ devices flashed with 9 and 15 pulses showed lower performances compared to the thermally annealed devices $\left(165{ }^{\circ} \mathrm{C}, 40 \mathrm{~min}\right.$ with mobility of $0.055 \mathrm{~cm}^{2} \mathrm{~V}^{-1} \mathrm{~s}^{-1}$ ), the TFTs showed typical n-type channel behavior similar to the thermally annealed samples with an increasing number of pulses from 30 to 45 . The electron mobility of $\mathrm{ZnO}$ films flashed with 30 pulses was comparable with that of the reference TFTs $\left(0.058 \mathrm{~cm}^{2} \mathrm{~V}^{-1} \mathrm{~s}^{-1}\right)$.

In a similar study conducted by the same group in 2016, IZO thin films were FLA treated with a high energy density of UV light $\left(250000 \mathrm{~J} \mathrm{~cm}^{-2}\right)$ on $\mathrm{Si} / \mathrm{SiN}_{x}$ substrate using different number of pulses ranging from 12 to 72 while the samples were kept on $120^{\circ} \mathrm{C}$ on a hot plate to remove the residual solvents. ${ }^{[239]}$ The reference sample was thermally annealed at $450{ }^{\circ} \mathrm{C}$ for $1 \mathrm{~h}$. Devices subjected to less than 51 pulses, showed poor performance in comparison with thermally annealed TFTs for which an electron mobility value of $2.72 \mathrm{~cm}^{2} \mathrm{~V}^{-1} \mathrm{~s}^{-1}$ was obtained. However, for TFTs subjected to 51 pulses, electron mobility of $2.38 \mathrm{~cm}^{2} \mathrm{~V}^{-1} \mathrm{~s}^{-1}$ was obtained; a value comparable to thermally annealed devices. When the number of pulses was increased to $>51$, the resulting TFT exhibited degraded performances.

Different from the previously discussed studies involving conventional IGZO precursors such as acetates or nitrates, Benwadih et al. used organo-metallic oxymates as the metal-oxide
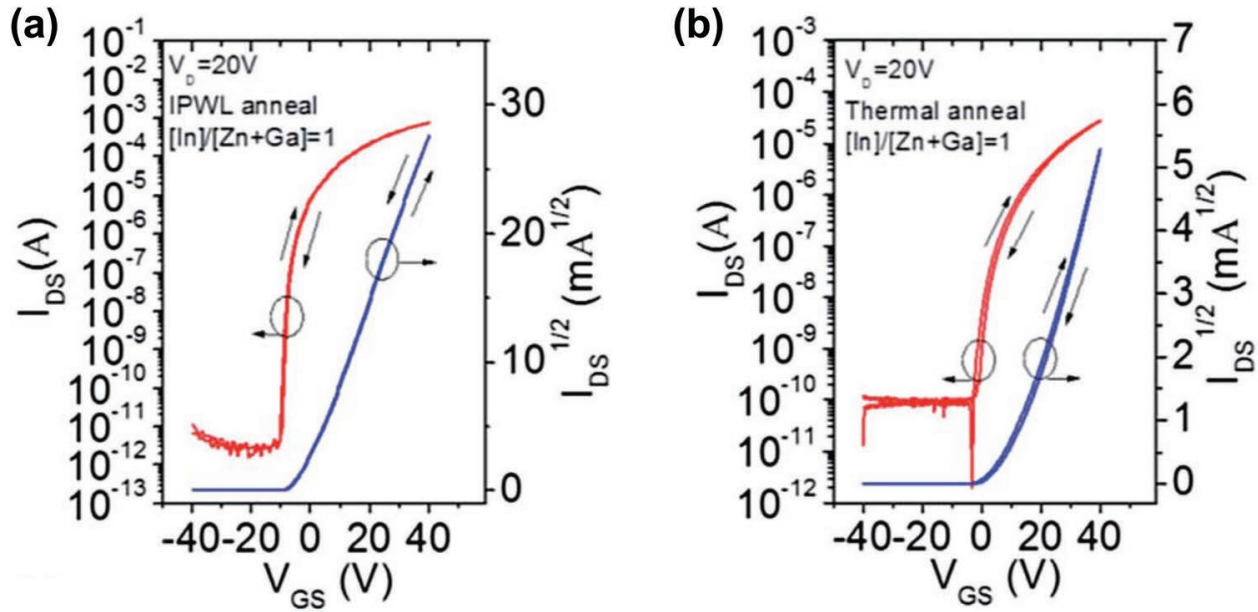

Figure 20. Transfer characteristic of a) FLA and b) thermally annealed IGZO TFTs. Reproduced with permission. ${ }^{[25]}$ Copyright 2014, RSC Publishing. 
(a)

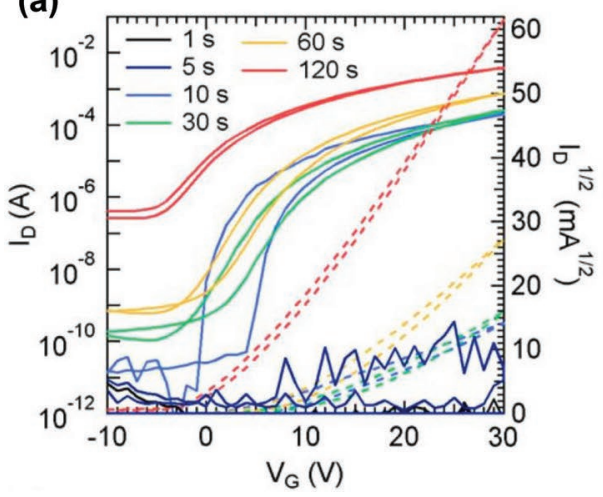

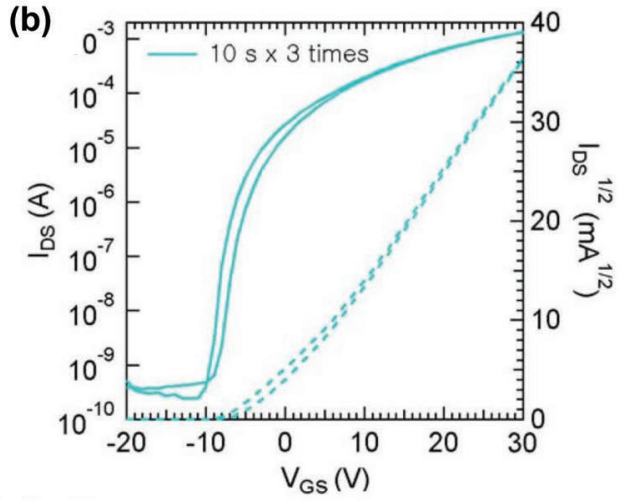

Figure 21. a) The transfer characteristics of $\ln _{2} \mathrm{O}_{3}$ TFTs with different FLA times from 1 to $120 \mathrm{~s}$ and b) $10 \mathrm{~s}$ with three repetitions. Reproduced with permission. ${ }^{[224]}$ Copyright 2016, IEEE.

precursors and exposed them to pulsed UV light to produce IGZO TFTs on $\mathrm{Si} / \mathrm{SiO}_{2}$ and polyimide substrates. ${ }^{[240]}$ IGZO precursor solution was spin-coated onto $\mathrm{Si} / \mathrm{SiO}_{2}$ substrates and baked at different temperatures ranging from 100 to $350{ }^{\circ} \mathrm{C}$ for $1 \mathrm{~min}$ followed by FLA treatment with radiant energies of $2000 \mathrm{~J}$ and 50 pulses with a pulse length of $200 \mu \mathrm{s}$. With increasing baking temperature, an increase of the electron mobility of the IGZO TFTs was observed with a maximum value of $7 \mathrm{~cm}^{2} \mathrm{~V}^{-1} \mathrm{~s}^{-1}$ for FLA treated devices baked at $350{ }^{\circ} \mathrm{C}$ (Figure 22a). TFTs baked at $300^{\circ} \mathrm{C}$ for $1 \mathrm{~min}$ without FLA did not show any transistor function.

In the second part of the study, UV pulse annealed IGZO TFTs were grown on polyimide substrates in a top-gate, bottomcontact architecture using PS/PVP (50 nm/350 nm) dielectric layers (Figure 22b,c). Compared to the thermally annealed sample which was baked at $350{ }^{\circ} \mathrm{C}$ for $1 \mathrm{~h}\left(3.8 \mathrm{~cm}^{2} \mathrm{~V}^{-1} \mathrm{~s}^{-1}\right.$, the FLA treated samples involved a short prebaking step at $350{ }^{\circ} \mathrm{C}$ for $1 \mathrm{~min}$ and showed improved electron mobility of $4.5 \mathrm{~cm}^{2} \mathrm{~V}^{-1} \mathrm{~s}^{-1}$ ).

The effect of FLA on the defect formation in IGZO TFTs was also examined by Eom et al. by analyzing the formation of nitrogen defects inside the IGZO layer. ${ }^{[242]}$ Spin-coated IGZO TFTs were fabricated with different irradiation times during FLA and different temperatures in the case of reference devices produced via thermal annealing. Similar trends were observed for either increased thermal annealing temperatures or increased flashing times, where the mobility was found to increase, and the channel current on/off ratio decrease. The result was attributed to the formation of defects leading to an increased concentration of free charge carriers. The TFTs flashed at a fire rate of $15 \mathrm{~Hz}$ with pulses featuring radiant energy of $207 \mathrm{~J}$ and a total irradiation time of $18 \mathrm{~s}$, showed a mobility value of $8.6 \mathrm{~cm}^{2} \mathrm{~V}^{-1} \mathrm{~s}^{-1}$ which was higher than TFTs annealed at $400{ }^{\circ} \mathrm{C}$ for $2 \mathrm{~h}\left(4.7 \mathrm{~cm}^{2} \mathrm{~V}^{-1} \mathrm{~s}^{-1}\right)$. It was observed that increasing exposure time caused increased off-current levels in the channel. To understand this behavior, elemental analysis via XPS was conducted to determine the effect of the treatment on the chemical composition of the channel layer. It was argued that for increasing FLA irradiation time, the concentration of nitrogen within the IGZO increased, with $\mathrm{N}_{2}$ taking up positions within oxygen vacancies. This particular type of defect behaves like an electron donor and increases the off-current level of IGZO TFTs.

Lee et al. demonstrated the FLA treatment of printed IGZO TFTs for the first time. ${ }^{[238]}$ Circular patterns were formed by microdispensing the precursor solution on a Si wafer followed by exposure to FLA with the radiant energy of $100 \mathrm{~J} \mathrm{~cm}^{-2}$. From one droplet to 3 droplets, the electron mobility of the devices increased from $0.9 \times 10^{-2}$ to $10.5 \times 10^{-2} \mathrm{~cm}^{2} \mathrm{~V}^{-1} \mathrm{~s}^{-1}$ due to the increase in the thickness of the IGZO channel. However, the use of more than three drops lead to a gradual mobility decay with values of $8 \times 10^{-2} \mathrm{~cm}^{2} \mathrm{~V}^{-1} \mathrm{~s}^{-1}$ for four drops and $5.3 \times 10^{-2} \mathrm{~cm}^{2} \mathrm{~V}^{-1} \mathrm{~s}^{-1}$ for five drops. The negative impact of the
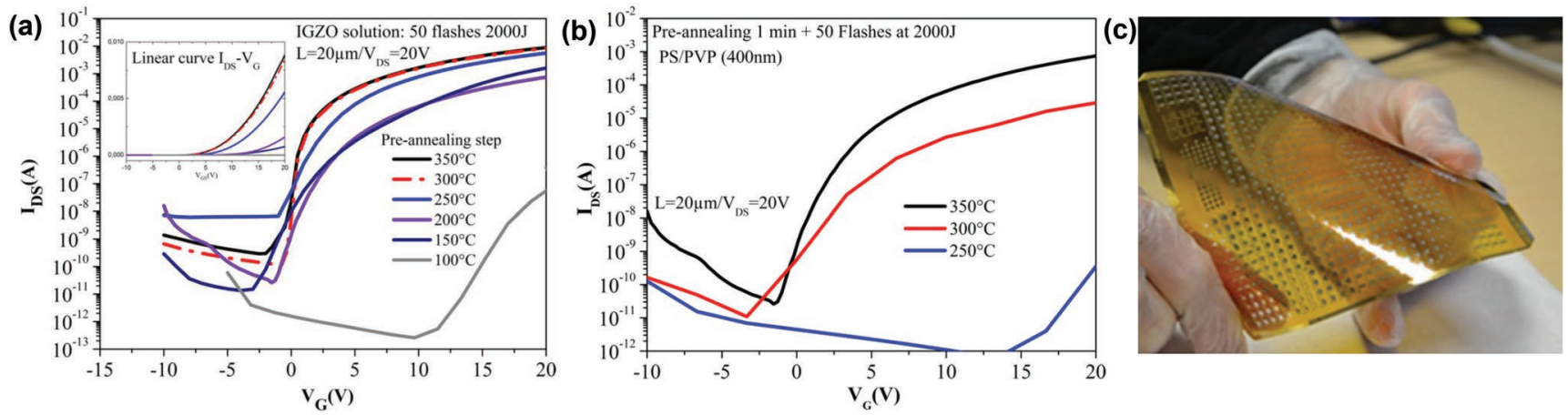

Figure 22. a) The effect of drying temperature $(1 \mathrm{~min})$ with the same FLA conditions on the transfer characteristics of ICZO TFTs. b) The transfer characteristics of metal-oxide TFTs prepared on PI using different drying temperature and the same UV FLA conditions. c) TFTs fabricated on flexible PI substrate via UV FLA. Reproduced with permission. ${ }^{[240]}$ Copyright 2016, American Chemical Society. 

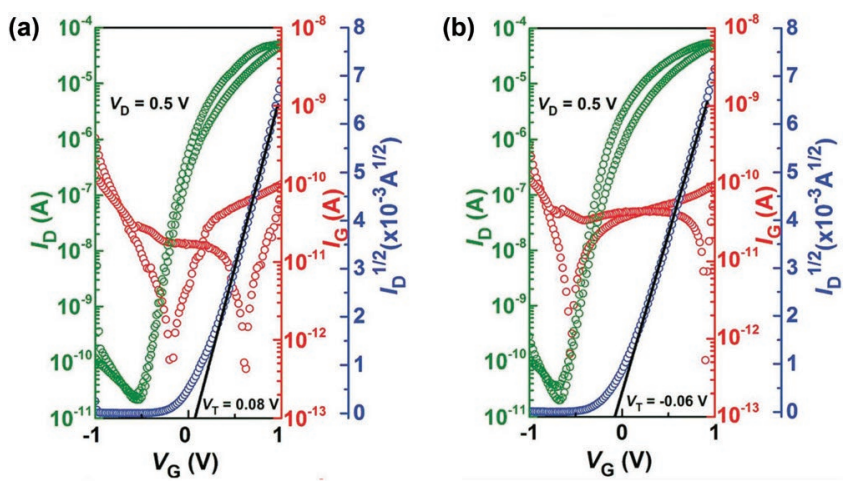

Figure 23. The transfer characteristics of printed $\ln _{2} \mathrm{O}_{3}$ treated with a) UVvis FLA and b) UVLA-laser annealing. Reproduced with permission. [241] Copyright 2017, Wiley-VCH.

increased number of drops was attributed to a change in the layer morphology.

Another printing study of metal-oxide TFTs comparing UV-vis FLA and UVLA treatment was conducted by Garlapati et al. using an indium oxide ink stabilized with poly (acrylic acid, sodium salt) ligand groups. ${ }^{[241]}$ As the gate dielectric, a composite solid polymer electrolyte was printed on the active channel to create a bottom-contact, top-gate transistors. It was found that both UV-vis FLA and UVLA were able to remove the stabilizer and increase the densification and electrical conduction of $\operatorname{In}_{2} \mathrm{O}_{3}$ nanoparticles, leading to electron mobilities of 8 and $12 \mathrm{~cm}^{2} \mathrm{~V}^{-1} \mathrm{~s}^{-1}$ for UV-vis FLA and UVLA, respectively (Figure 23a,b). Even though the mobility of UVLA was slightly higher, the authors argued that, in terms of total set-up cost, the UV-vis FLA might be a better choice for roll-to-roll manufacturing.

The application of the FLA technique on solution-processed heterojunction metal-oxide semiconductors on glass substrates was first reported by Tetzner et al. ${ }^{[22]}$ The authors combined thin-films of $\mathrm{In}_{2} \mathrm{O}_{3}$ and $\mathrm{ZnO}$ deposited sequentially via spincoating and dried at $130{ }^{\circ} \mathrm{C}$ followed by exposure of each layer to 20 light flashes at an energy density of $5 \mathrm{~J} \mathrm{~cm}^{-2}$ and a pulse length of $500 \mu \mathrm{s}$. In order to achieve an adequate temperature for the sol-gel conversion, the researchers introduced a thick $\mathrm{Al}$ gate electrode $(100 \mathrm{~nm})$ that absorbed part of the radiant energy. This was necessary since the material stack was composed of a highly transparent substrate and the semiconductor precursor layers. High charge carrier mobilities of 6 and $36 \mathrm{~cm}^{2} \mathrm{~V}^{-1} \mathrm{~s}^{-1}$ were achieved for $\mathrm{In}_{2} \mathrm{O}_{3}$ single layers and $\mathrm{In}_{2} \mathrm{O}_{3} / \mathrm{ZnO}$ heterojunction layers, respectively. The performances of these FLA treated devices were comparable to devices that were thermally annealed at $250^{\circ} \mathrm{C}$ for $1 \mathrm{~h}$. Furthermore, using numerical calculations, the peak temperature was found to be almost $1000^{\circ} \mathrm{C}$ on the top surface after each pulse due to the high absorption of the xenon flash in the gate metal and the low thermal conductivity of the glass substrate.

More recently, a two steps flashlight annealing was conducted by Moon et al. for the very first time, employing solution processed IGZO layers. ${ }^{[244]}$ They combined a short-time NIR or DUV/NIR drying step with a white light FLA as an alternative method to conventional hot plate drying. This shorttime photodrying step gave rise to the decomposition of organic residues and enhance the hydrolysis of the solution with photochemical reaction. The samples solely dried with NIR light within $1 \mathrm{~min}$ did not show any transistor behavior. However, when the dried films were flashed under different FLA irradiation energies, the performance of TFTs increased with maximum electron mobility of $13.5 \mathrm{~cm}^{2} \mathrm{~V}^{-1} \mathrm{~s}^{-1}$ and a low on/off ratio of around $10^{4}$ (Figure 24a). To increase the low on/off ratio, an additional drying step with DUV light was performed prior to the high-intensity FLA. Although there was also a small decrease in the on-current, the off-current showed ten times less current, as seen in Figure 24b. In order to better understand the effect of DUV treatment, sol-gel films were dried under different DUV intensities without changing the conditions of the NIR light exposure followed by the high-intensity flash annealing. Optimum conditions were found at a DUV intensity of $60 \mathrm{~mW} \mathrm{~cm}$ cr $^{-2}$ resulting in improved on/off current ratios around $10^{6}$ and electron mobilities of $7.7 \mathrm{~cm}^{2} \mathrm{~V}^{-1} \mathrm{~s}^{-1}$. Further increase of the DUV intensity caused a reduction of the on/off current ratio due to an increase of the electron concentration and an increased off channel current (Figure 24c).

\section{Conclusions and Perspectives}

We have reviewed the recent progress in the field of photonic processing of metal-oxide materials for the manufacturing of modern large-area electronics on a variety of substrate
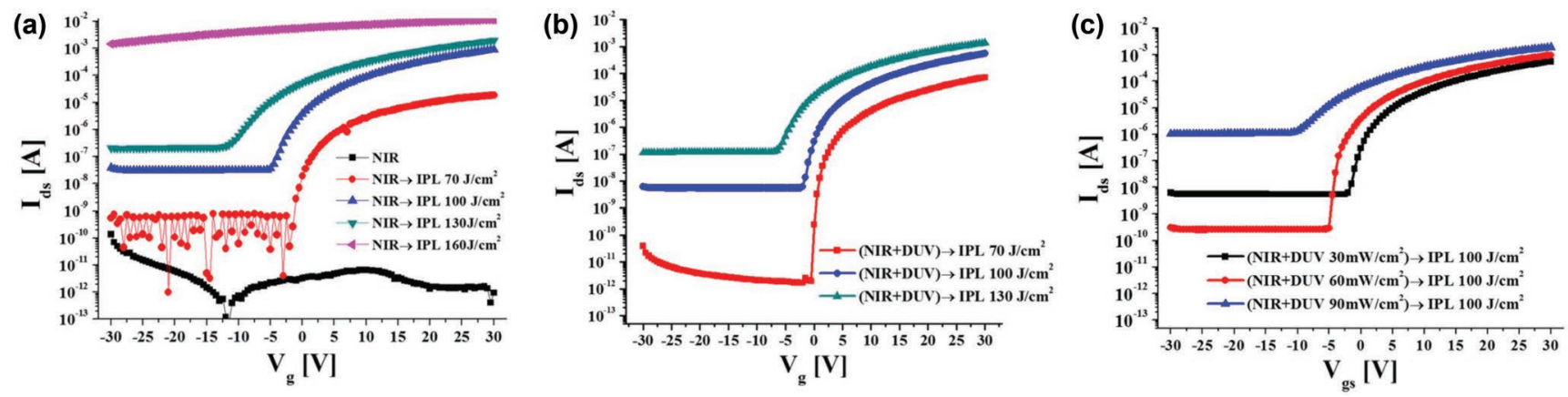

Figure 24. The transfer characteristics of IGZO TFTs subjected to: a) 1 min NIR photodrying followed by FLA with different energies; b) 1 min NIR and DUV photodrying followed by FLA with different energies; c) drying with different DUV energies with the same NIR and FLA energies. Reproduced with permission. ${ }^{[244]}$ Copyright 2019, American Chemical Society. 
Table 5. SWOT analysis table for the processing of metal-oxide layers and devices via deepultraviolet (DUV) light illumination.

\begin{tabular}{|c|c|}
\hline Strengths & Weaknesses \\
\hline $\begin{array}{l}\text { - Low capital investment } \\
\text { - Simple setup and maintenance } \\
\text { - Scalability } \\
\text { - Ability to initiate photochemical reactions }\end{array}$ & $\begin{array}{l}\text { - Longer treatment times compared to other } \\
\text { optical processes } \\
\text { - Precursor layers may need to be photosensitized }\end{array}$ \\
\hline Opportunities & Threats \\
\hline $\begin{array}{l}\text { - Large-area electronics } \\
\text { - Possibility of direct patterning with photomasks } \\
\text { - Combining DUV and thermal annealing lowers } \\
\text { annealing temperature and treatment time } \\
\text { significantly }\end{array}$ & $\begin{array}{l}\text { - Unintentional heating due to long exposure times } \\
\text { (RT to } 150^{\circ} \mathrm{C} \text { ) } \\
\text { - Possible damage to other circuit components } \\
\text { during processing } \\
\text { - Incompatible with R2R and/or S2S processing due } \\
\text { to long treatment time }\end{array}$ \\
\hline
\end{tabular}

using a photomask. This approach can potentially reduce the number of process steps and allows for more environmentally friendly etching solutions when compared to standard lithography. Despite its simplicity, the DUV treatment of metal oxides can prove to be highly effective when implemented under the appropriate conditions.

Across the existing annealing methodologies, significant progress has been accomplished over the past decades toward faster ramp rates and shorter high-temperature cycle times, from $\approx 1 \mathrm{~s}$ in rapid thermal annealing to $<1 \mathrm{~ms}$ for FLA and LA. In particular, pulsed lasers offer ultrafast interaction of few ns $(\approx 20 \mathrm{~ns})$, which help to materials. The various light source technologies including lasers, ultraviolet lamps and flash lamps, have been thoroughly reviewed with emphasis on their suitability for direct conversion and postprocessing of precursors and predeposited metal oxides, respectively, and their practical utilization in transistors and microelectronic applications.

Despite the early days, the strides made toward photonically processed oxide materials and devices are impressive and highlight the potential for further technological developments toward a transformative manufacturing paradigm that combines intriguing features such as scalability and low-cost, with the prospect for drastically reduced manufacturing time. With this in mind, we briefly highlight pros and cons of each technique in the form of strengths, weaknesses, opportunities, and threats (SWOT).

Among the various techniques discussed here, DUV illumination (Table 5) is by far the one that requires the lowest capital investment and is the simplest to implement. The energy intake to the sample is often controlled via the duration of illumination since the energy density is relatively low. As a result, sample treatment typically occurs over tens of minutes, making DUV the technique that requires the longest processing times among those discussed here, resulting in relatively low throughput fabrication. Unless actively controlled, the substrate tends to heat up unintentionally during DUV treatment, with highest reported temperature of up to $150{ }^{\circ} \mathrm{C}$. While this is still acceptable for the use with many common polymer substrates, it may be high enough to damage other device components. On the other hand, when DUV is combined with simultaneous TA, the required process times and/or process temperature are both found to reduce significantly as compared to TA alone. When DUV is combined with solution processed precursor formulations, their UV absorption capability has to be guaranteed in order to assure material photoconversion. Although the addition of photosensitizers may lead to an increased concentration of impurities in the metal oxide, it certainly opens up the DUV treatment to a wider range of candidate materials, while simultaneously facilitating the option to directly pattern the metal oxide decouple important physical phenomena. Laser processing is one of the most precise industrial processing tools on the market and has many applications, of which semiconductor lithography is perhaps the best known. ${ }^{[248]}$ By the same token LA represent a promising technology in the field of metal-oxide electronics (Table 6). LA combines fast processing with rapid, precise and selective energy delivery via critical photon energy absorption by the targeted layer while leaving the substrate intact. This feature makes it compatible with important temperature sensitive substrate materials such as plastics, while its ultrafast nature makes it attractive for R2R applications. To this end, the use of lasers can provide multiple functionalities such as scribing, cutting, marking and ablation which can facilitate the fabrication of vertical interconnect access as well as the removal of material(s). Moreover, the high spatial resolution of LA gives accurate control over the processing area for selective annealing and/or patterning thus providing freedom of design triggering the digital transformation of TFT manufacturing.

However, like many other emerging technologies, LA comes with its own limitations and challenges that need to be addressed before widespread adoption by industry. From a fundamental point of view, temperature gradients induced by LA may cause thermal stresses caused by differential thermal expansion between the substrate and overlayer(s) of other materials. Although these stresses are typically localized to avoid shattering, other stress-induced effects may still cause different
Table 6. SWOT analysis table for the processing of metal-oxide layers and devices via laser annealing (LA).

\begin{tabular}{ll}
\hline Strengths & Weaknesses \\
\hline - Scalable/adaptable by industry & - Cost (capital, operational, maintenance) \\
- Simultaneous patterning and Annealing & - Complex design (optical delivery path, scanning \\
- Material and area selective & optical elements) \\
- Precise energy delivery (ultrafast) & - Serial process (scanning beam) \\
- Low temperature process & - Developing knowledge on fundamentals \\
- Digital manufacturing technology & (photochemistry, chemical processes) \\
\hline Opportunities & \multicolumn{1}{c}{ Threats } \\
\hline - Large-area electronics & - Light source instability (energy delivery fluctuation) \\
- Multi-functionality (e.g., scribing, cutting, marking, & - Long lead times (for light sources) \\
ablating, formation of vertical interconnects) & - Compatibility with other device components \\
& - Limited technological maturity for applications on \\
& metal oxides \\
\hline
\end{tabular}


defects. Considering its ns scale interaction, LA is inherently a nonequilibrium processes. Dopants may be frozen in thermodynamically metastable states that may permit diffusion and deactivation during subsequent thermal exposure later in the process flow. In addition, optical pattern effects can be difficult to overcome (layers may absorb, refract, or diffract the laser light in complicated ways). Such effects can cause nonuniform thermal exposure at the individual device level.

From a practical point of view, the cost of ownership would be a prime consideration whenever a laser system is considered for installation and use. Systems based on excimer lasers can be expected to be at the higher end of this price range, whereas systems such as Nd:YAG lasers can be expected at the lower end. Operational and maintenance costs would include consumables like optics as well as online diagnostics (CAD software, sensors and controllers with loop feedback), specialist components and consumables (thyratron and gasses for excimer lasers, flash lamp and lasing rods for Nd:YAG lasers), but also further auxiliary systems may be required like cooling systems and safety equipment like high power UV light protection and local extraction and ventilation systems with suitable filtration for gasses and/or particulates.

Despite LA's potential and the evident wealth of experimental data, there is still a lack of knowledge and in-depth understanding on the effects of laser processing of metal oxides. In addition, the mechanism behind the precursor's chemical conversion and the condensation and densification of the metal oxides induced via LA is still not fully understood. To overcome this roadblock a significant effort needs to be invested in the theoretical exploration of the underlying physical and chemical mechanisms and a multi-scale approach should be adapted to gain the essential knowledge to determine and clarify the above mentioned research questions. Moreover, the relative merits of combining different annealing methods (e.g., furnace or hot plate annealing followed by laser irradiation) lacks clear physical explanation and progress has been largely based on experimental studies, that are inherently limited in covering the full parameter space of such a multi-parametric process like LA.

When compared to DUV, FLA offers an alternative photonic-based technique suitable for metal-oxide processing (Table 7). Unlike DUV however, FLA offers rapid processing times, and as such high throughput rates, but without compromising scalability. The usage of the full spectrum of high

Table 7. SWOT analysis table for the processing of metal-oxide layers and devices via flash lamp annealing FLA.

\begin{tabular}{ll}
\hline Strengths & Weaknesses \\
\hline $\begin{array}{ll}\text { - Highly scalable and adaptable for industrial } \\
\text { applications }\end{array}$ & $\begin{array}{l}\text { - Inefficient energy delivery to the material under } \\
\text { processing }\end{array}$ \\
- High energy supply (broad spectrum) & - Stress formation in films during treatment \\
- Low temperature processing & - Possible need for complex buffer layer(s) \\
- Suitable for R2R and S2S fabrication & - Possible need for light-absorbing elements \\
\hline Opportunities & Threats \\
\hline - Large area electronics & - Light source stability (energy delivery fluctuation) \\
- High throughput rates and fast processing times & - Possible damage to other device/circuit \\
suitable for industrial applications & components \\
- Tunable light spectrum via optical filters & - Limited technological maturity \\
\hline
\end{tabular}

intensity xenon flash lamps combined with appropriate driving electronics enables the delivery of high energy to the material under processing, which can result in high temperature on the surface of the treated layer while keeping the substrate at RT.

Since the majority of metal oxides relevant to electronics are wide bandgap materials, they tend to absorb only a small amount of the total optical energy delivered by the flash lamp. One approach often used to compensate for this loss is to incorporate additional absorptive elements within the treated sample (or structure). Unfortunately, this increases the possibility of mismatch in the thermal coefficient of expansion (TCE) between the different layers (oxides and absorptive elements) resulting in film deformation due to stresses. A possible mitigation strategy is the incorporation of suitable buffer layer(s) that assure structural integrity, but this increases complexity and potentially cost.

Other challenges associated with the use of FLA in R2R manufacturing processes include the long term stability of the flash lamp, potential nonuniformly distributed light output as well as the requirement to protect pre-existing device components from exposure to intense light. Although still at its infancy, FLA combines numerous attractive features making it an attractive option for the rapid processing of metal oxides as well as other key device components such as metal inks.

Overall, photonic processing appears to be unique in decoupling the high thermal budget, often required for the synthesis of high-quality metal oxides, from the thermal properties of the substrate material. Such capabilities are becoming increasingly important in the broader area of large-area electronics due to the continuously increasing use of temperature-sensitive substrate materials, such as polymers, and the associated processing restrictions imposed by the narrow thermal budget tolerances. It has also become evident that photonic processing routes could increase the overall speed of manufacturing since the latter depends on a trade-off between the physical processes involved during material conversion or treatment, and quality/ performance of the targeted materials and devices. Considering the advantages and disadvantages of the various photonic processes discussed and analyzed here, the best approach may not necessarily rely on the use of a single photonic technique, but rather on a combination of two or more. For instance, one could imagine cases where an initial FLA process step is followed up by a precise and spatially selective LA etc.

As the various photonic processes and materials continue to evolve, the prospects for all photonically processed oxide electronics are slowly becoming a reality. In order for this vision, however, to fully materialize a radical transition from laboratory scale experimental setups to precommercial production systems that employ the new photonic-based process technologies would be required. This review aims to consolidate all critical aspects of photonic processing of metaloxide electronics with a primary aim to highlight the potential but also technical challenges and practical solutions reported in the literature. The interested reader may thus use this material either as an introduction to photonic processing of oxides or as a source for further study for large-area electronics manufacturing. 


\section{Acknowledgements}

The authors acknowledge King Abdullah University of Science and Technology (KAUST) for the financial support.

\section{Conflict of Interest}

The authors declare no conflict of interest.

\section{Keywords}

flash lamps, laser processing, metal oxides, photonic processing, thinfilm transistors

Received: July 24, 2019

Revised: September 19, 2019

Published online:

[1] E. Fortunato, P. Barquinha, R. Martins, Adv. Mater. 2012, 24, 2945.

[2] S. J. Kim, S. Yoon, H. J. Kim, Jpn. J. Appl. Phys. 2014, 53, 02 BA02.

[3] S. Hong, J. W. Park, H. J. Kim, Y. G. Kim, H. J. Kim, J. Inf. Disp. 2016, 17, 93.

[4] B. Du Ahn, H.-J. Jeon, J. Sheng, J. Park, J.-S. Park, Semicond. Sci. Technol. 2015, 30, 064001.

[5] J. S. Park, W.-J. Maeng, H.-S. Kim, J.-S. Park, Thin Solid Films 2012 , 520, 1679.

[6] S. R. Thomas, P. Pattanasattayavong, T. D. Anthopoulos, Chem. Soc. Rev. 2013, 42, 6910

[7] E. M. C. Fortunato, P. M. C. Barquinha, A. C. M. B. G. Pimentel, A. M. F. Gonçalves, A. J. S. Marques, L. M. N. Pereira, R. F. P. Martins, Adv. Mater. 2005, 17, 590.

[8] Y. Sun, J. A. Rogers, Adv. Mater. 2007, 19, 1897.

[9] Z. Fan, D. Wang, P.-C. Chang, W.-Y. Tseng, J. G. Lu, Appl. Phys. Lett. 2004, 85, 5923.

[10] I. Isakov, H. Faber, M. Grell, G. Wyatt-Moon, N. Pliatsikas, T. Kehagias, G. P. Dimitrakopulos, P. P. Patsalas, R. Li, T. D. Anthopoulos, Adv. Funct. Mater. 2017, 27, 1606407.

[11] D. Khim, Y.-H. Lin, S. Nam, H. Faber, K. Tetzner, R. Li, Q. Zhang, J. Li, X. Zhang, T. D. Anthopoulos, Adv. Mater. 2017, 29, 1605837.

[12] B. Wang, W. Huang, L. Chi, M. Al-Hashimi, T. J. Marks, A. Facchetti, Chem. Rev. 2018, 118, 5690.

[13] K. Myny, Nat. Electron. 2018, 1, 30.

[14] X. Yu, T. J. Marks, A. Facchetti, Nat. Mater. 2016, 15, 383.

[15] G. V. Naik, V. M. Shalaev, A. Boltasseva, Adv. Mater. 2013, 25, 3264.

[16] M. W. J. Prins, K. O. Grosse-Holz, G. Müller, J. F. M. Cillessen, J. B. Giesbers, R. P. Weening, R. M. Wolf, Appl. Phys. Lett. 1996, $68,3650$.

[17] Z. Wang, P. K. Nayak, J. A. Caraveo-Frescas, H. N. Alshareef, Adv. Mater. 2016, 28, 3831.

[18] Y.-H. Kim, J.-S. Heo, T.-H. Kim, S. Park, M.-H. Yoon, J. Kim, M. S. Oh, G.-R. Yi, Y.-Y. Noh, S. K. Park, Nature 2012, 489, 128.

[19] A. Liu, H. Zhu, H. Sun, Y. Xu, Y.-Y. Noh, Adv. Mater. 2018, 30, 1706364.

[20] W. Xu, H. Li, J. Xu, L. Wang, ACS Appl. Mater. Interfaces 2018, 10, 25878.

[21] K. K. Banger, Y. Yamashita, K. Mori, R. L. Peterson, T. Leedham, J. Rickard, H. Sirringhaus, Nat. Mater. 2011, 10, 45.

[22] M. G. Kim, M. G. Kanatzidis, A. Facchetti, T. J. Marks, Nat. Mater. 2011, 10, 382.
[23] Y.-H. Lin, H. Faber, K. Zhao, Q. Wang, A. Amassian, M. McLachlan, T. D. Anthopoulos, Adv. Mater. 2013, 25, 4340.

[24] T. Jun, K. Song, Y. Jeong, K. Woo, D. Kim, C. Bae, J. Moon, J. Mater. Chem. 2011, 21, 1102.

[25] T. H. Yoo, S. J. Kwon, H. S. Kim, J. M. Hong, J. A. Lim, Y. W. Song, RSC Adv. 2014, 4, 19375

[26] T. Nagase, T. Ooie, J. Sakakibara, Thin Solid Films 1999, 357, 151.

[27] Y. S. Rim, H. Chen, Y. Liu, S. H. Bae, H. J. Kim, Y. Yang, ACS Nano 2014, 8, 9680

[28] D. V Bellas, D. Toliopoulos, N. Kalfagiannis, A. Siozios, P. Nikolaou, P. C. Kelires, D. C. Koutsogeorgis, P. Patsalas, E. Lidorikis, Thin Solid Films 2017, 630, 7.

[29] M. J. Guillot, K. A. Schroder, S. C. Mccool, in ASME 2012 International Mechanical Engineering Congress \& Exposition IMECE2012, ASME, New York, USA 2012, pp. 19-27.

[30] Y. M. Park, J. Daniel, M. Heeney, A. Salleo, Adv. Mater. 2011, 23, 971.

[31] D. Vak, K. Hwang, A. Faulks, Y.-S. Jung, N. Clark, D.-Y. Kim, G. J. Wilson, S. E. Watkins, Adv. Energy Mater. 2015, 5, 1401539.

[32] W. J. Scheideler, M. W. McPhail, R. Kumar, J. Smith, V. Subramanian, ACS Appl. Mater. Interfaces 2018, 10, 37277.

[33] Y. M. Park, A. Desai, A. Salleo, L. Jimison, Chem. Mater. 2013, 25, 2571.

[34] J. Leppäniemi, K. Ojanperä, T. Kololuoma, O.-H. Huttunen, J. Dahl, M. Tuominen, P. Laukkanen, H. Majumdar, A. Alastalo, Appl. Phys. Lett. 2014, 105, 113514.

[35] I. W. Boyd, J.-Y. Zhang, Solid-State Electron. 2001, 45, 1413.

[36] K. Umeda, T. Miyasako, A. Sugiyama, A. Tanaka, M. Suzuki, E. Tokumitsu, T. Shimoda, J. Appl. Phys. 2013, 113, 184509.

[37] X. Dong, G. Xia, Q. Zhang, L. Li, H. Gong, J. Bi, S. Wang, Ceram. Int. 2017, 43, 15205 .

[38] B.-Y. Su, S.-Y. Chu, Y.-D. Juang, H.-C. Chen, Appl. Phys. Lett. 2013, 102, 192101.

[39] Y. J. Tak, B. Du Ahn, S. P. Park, S. J. Kim, A. R. Song, K. B. Chung, H. J. Kim, Sci. Rep. 2016, 6, 21869.

[40] Y. S. Rim, H. S. Lim, H. J. Kim, ACS Appl. Mater. Interfaces 2013, $5,3565$.

[41] E. Carlos, R. Branquinho, A. Kiazadeh, P. Barquinha, R. Martins, E. Fortunato, ACS Appl. Mater. Interfaces 2016, 8, 31100.

[42] K.-H. Wang, H.-W. Zan, O. Soppera, Semicond. Sci. Technol. 2018, 33, 035003.

[43] H. S. Lim, Y. S. Rim, H. J. Kim, Sci. Rep. 2014, 4, 4544.

[44] K. K. Banger, R. L. Peterson, K. Mori, Y. Yamashita, T. Leedham, H. Sirringhaus, Chem. Mater. 2014, 26, 1195.

[45] M. L. Calzada, A. González, R. Poyato, L. Pardo, J. Mater. Chem. 2003, 13, 1451

[46] W. Lee, S. Choi, J. Kim, S. K. Park, Y. H. Kim, Adv. Electron. Mater. 2019, 5, 1900073

[47] S. Ogura, H. Cheong, S. Uemura, H. Ushijima, N. Fukuda, Flexible Printed Electron. 2016, 1, 045001.

[48] R. A. John, N. A. Chien, S. Shukla, N. Tiwari, C. Shi, N. G. Ing, N. Mathews, Chem. Mater. 2016, 28, 8305.

[49] J. Hwang, J. Park, H. Kim, Semicond. Sci. Technol. 2014, 29, 095019.

[50] J. Hwang, K. Lee, Y. Jeong, Y. U. Lee, C. Pearson, M. C. Petty, H. Kim, Adv. Mater. Interfaces 2014, 1, 1400206.

[51] S. Park, K.-H. Kim, J.-W. Jo, S. Sung, K.-T. Kim, W.-J. Lee, J. Kim, H. J. Kim, G.-R. Yi, Y.-H. Kim, M.-H. Yoon, S. K. Park, Adv. Funct. Mater. 2015, 25, 2807.

[52] Y.-H. Lin, H. Faber, S. Rossbauer, T. D. Anthopoulos, Appl. Phys. Lett. 2016, 102, 193516.

[53] Y. J. Jeong, T. K. An, D. J. Yun, L. H. Kim, S. Park, Y. Kim, S. Nam, K. H. Lee, S. H. Kim, J. Jang, C. E. Park, ACS Appl. Mater. Interfaces 2016, 8, 5499.

[54] J. H. Kim, Y. S. Rim, H. J. Kim, ACS Appl. Mater. Interfaces 2014, 6, 4819 . 
[55] T. II Lee, H. K. Baik, J. H. Park, J. H. Lee, Y. B. Yoo, S. S. Chae, Chem. Phys. Lett. 2014, 597, 121.

[56] N. Tohge, K. Shinmou, T. Minami, J. Sol-Gel Sci. Technol. 1994, 2, 581.

[57] K. Shinmou, N. Tohge, T. Minami, Jpn. J. Appl. Phys. 1994, 33, L1181.

[58] T. Kololuoma, A. H. O. Kärkkäinen, A. Tolonen, J. T. Rantala, Thin Solid Films 2003, 440, 8426.

[59] Y. Li, G. Zhao, W. Zhang, Y. Chen, J. Disp. Technol. 2006, 2, 175.

[60] T. Ohishi, S. Maekawa, A. Katoh, J. Non-Cryst. Solids 1992, 147-148, 493

[61] T. B. Daunis, D. Barrera, G. Gutierrez-Heredia, O. Rodriguez-Lopez, J. Wang, W. E. Voit, J. W. P. Hsu, J. Mater. Res. 2018, 33, 2454.

[62] E. Carlos, R. Branquinho, A. Kiazadeh, J. Martins, P. Barquinha, R. Martins, E. Fortunato, ACS Appl. Mater. Interfaces 2017, 9, 40428.

[63] J. S. Heo, J. H. Kim, J. Kim, M. G. Kim, Y. H. Kim, S. K. Park, IEEE Electron Device Lett. 2015, 36, 162.

[64] W. Lee, J. Kim, Y. H. Kim, J. Alloys Compd. 2017, 723, 627.

[65] H. J. Kim, Y. Kim, S. P. Park, D. Kim, N. Kim, J. S. Choi, H. J. Kim, SID Symp. Dig. Tech. Pap. 2017, 48, 180.

[66] J.-W. Jo, J. Kim, K.-T. Kim, J.-G. Kang, M.-G. Kim, K.-H. Kim, H. Ko, Y.-H. Kim, S. K. Park, Adv. Mater. 2015, 27, 1182.

[67] Y.-H. Lin, H. Faber, J. G. Labram, E. Stratakis, L. Sygellou, E. Kymakis, N. A. Hastas, R. Li, K. Zhao, A. Amassian, N. D. Treat, M. McLachlan, T. D. Anthopoulos, Adv. Sci. 2015, 2, 1500058

[68] T. B. Singh, J. J. Jasieniak, L. De Oliveira Tozi, C. D. Easton, M. Bown, IEEE Trans. Electron Devices 2014, 61, 1093.

[69] X. Xu, Q. Cui, Y. Jin, X. Guo, Appl. Phys. Lett. 2012, 101, 222113.

[70] M. Miyakawa, M. Nakata, H. Tsuji, Y. Fujisaki, Sci. Rep. 2018, 8, 12825.

[71] W. T. Park, Y. Y. Noh, J. Mater. Chem. C 2017, 5, 6467.

[72] S. Sanctis, R. C. Hoffmann, M. Bruns, J. J. Schneider, Adv. Mater. Interfaces 2018, 5, 1800324.

[73] H. J. Wu, N. Tomiyama, H. Nagai, M. Sato, J. Cryst. Growth 2019, 509, 112.

[74] S. Bolat, P. Fuchs, S. Knobelspies, O. Temel, G. T. Sevilla, E. Gilshtein, C. Andres, I. Shorubalko, Y. Liu, G. Tröster, A. N. Tiwari, Y. E. Romanyuk, Adv. Electron. Mater. 2019, 5, 1800843.

[75] H. J. Seul, H. G. Kim, M. Y. Park, J. K. Jeong, J. Mater. Chem. C 2016, 4, 10486.

[76] S. K. Park, Y.-H. Kim, J.-I. Han, J. Phys. D: Appl. Phys. 2009, 42, 125102.

[77] H.-W. Zan, W.-T. Chen, C.-W. Chou, C.-C. Tsai, C.-N. Huang, H.-W. Hsueh, Electrochem. Solid-State Lett. 2010, 13, H144.

[78] J. L. Wu, H. Y. Lin, P. H. Kuo, B. Y. Su, S. Y. Chu, Y. C. Chen, S. Y. Liu, C. C. Chang, C. J. Wu, IEEE Trans. Electron Devices 2014, 61, 1403.

[79] L. Lu, M. Echizen, T. Nishida, Y. Ishikawa, K. Uchiyama, Y. Uraoka, AIP Adv. 2012, 2, 032111.

[80] H. Seo, C. Young-Je, K. Jinwoo, S. M.bobade, K.-Y. Park, D. Choi, J. Lee, Appl. Phys. Lett. 2010, 96, 222101.

[81] D. Weber, S. Botnaras, D. V. Pham, J. Steiger, L. De Cola, J. Mater. Chem. C 2013, 1, 3098

[82] S. Sung, S. Park, S. Cha, W. J. Lee, C. H. Kim, M. H. Yoon, RSC Adv. 2015, 5, 38125

[83] J. M. Fairfield, G. H. Schwuttke, Solid-State Electron. 2002, 11, 1175.

[84] J. C. Bean, H. J. Leamy, J. M. Poate, G. A. Rozgonyi, J. P. Van Der Ziel, J. S. Williams, G. K. Celler, J. Appl. Phys. 1979, 50, 881.

[85] M. Hatano, S. Moon, M. Lee, K. Suzuki, C. P. Grigoropoulos, J. Non-Cryst. Solids 2003, 266-269, 654.

[86] Y. Tamminga, G. E. J. Eggermont, W. K. Hofker, D. Hoonhout, R. Garrett, F. W. Saris, Phys. Lett. A 1979, 69, 436.
[87] M. Birnbaum, T. L. Stocker, J. Appl. Phys. 1968, 39, 6032.

[88] R. F. Wood, G. E. Giles, Phys. Rev. B 1981, 23, 2923.

[89] T. Sameshima, S. Usui, M. Sekiya, IEEE Electron Device Lett. 2008, 7, 276

[90] M. Cervera, B. J. Garcia, J. Martinez, J. Garrido, J. Piqueras, J. Appl. Phys. 1988, 64, 3079.

[91] H. Tsukamoto, T. Suzuki, Solid-State Electron. 1998, 42, 547.

[92] N. F. Borrelli, C. M. Smith, D. C. Allan, Opt. Lett. 1999, 24, 1401.

[93] M. Hatano, S. Moon, M. Lee, K. Suzuki, C. P. Grigoropoulos, J. Appl. Phys. 2000, 87, 36.

[94] H. Hosono, M. Kurita, H. Kawazoe, Thin Solid Films 1999, 351, 137.

[95] H. Hosono, M. Kurita, H. Kawazoe, Jpn. J. Appl. Phys. 1998, 37, L1119.

[96] S. Takeda, Y. Ikuta, M. Hirano, H. Hosono, J. Mater. Res. 2001, 16, 1003.

[97] D. Basting, K. D. Pippert, U. Stamm, in Proc. SPIE 4426, Second International Symposium on Laser Precision Microfabrication, SPIE, Bellingham, USA 2002, pp. 25-34.

[98] S. Cha, S. Lee, J. Eun Jang, A. Jang, J. Pyo Hong, J. Lee, J. Inn Sohn, D. Joon Kang, J. Min Kim, Appl. Phys. Lett. 2013, 103, 053114.

[99] S. Dellis, I. Isakov, N. Kalfagiannis, K. Tetzner, T. D. Anthopoulos, D. C. Koutsogeorgis, J. Mater. Chem. C 2017, 5, 3673.

[100] H. I. Shin, K. H. Kim, T. W. Kim, H. K. Kim, Ceram. Int. 2016, 42, 13983.

[101] P. Fan, Z. Sun, G. C. Wilkes, M. C. Gupta, Appl. Surf. Sci. 2019, 480, 35.

[102] L. Parellada-Monreal, I. Castro-Hurtado, M. Martínez-Calderón, L. Presmanes, G. G. Mandayo, Appl. Surf. Sci. 2019, 476, 569.

[103] S. Lauzurica, M. Lluscà, D. Canteli, M. I. Sánchez-Aniorte, J. López-Vidrier, S. Hernández, J. Bertomeu, C. Molpeceres, Proc. SPIE 2014, 9180, 918006.

[104] S. Heo, M. Chang, Y. Ju, S. Jung, H. Hwang, Appl. Phys. Lett. 2008, 93, 172115.

[105] G. R. Lin, C. J. Lin, Appl. Phys. Lett. 2007, 91, 072103.

[106] M. Li, W. Anderson, N. Chokshi, R. L. DeLeon, G. Tompa, J. Appl. Phys. 2006, 100, 053106.

[107] G. Dubourg, M. Radović, ACS Appl. Mater. Interfaces 2019, 11, 6257.

[108] X. Liu, W. Kuang, H. Ni, Z. Tao, J. Chang, Q. Liu, J. Ge, C. Li, Q. Dai, Small 2018, 14, 1800265.

[109] K. M. Yu, H. M. Ji, M. C. Nguyen, A. H. T. Nguyen, S. J. Choi, J. G. Cheon, J. H. Kim, S. W. Kim, S. Y. Cho, R. Choi, IEEE Electron Device Lett. 2019, 40, 167

[110] K. Siuzdak, M. Szkoda, M. Sawczak, J. Karczewski, J. Ryl, A. Cenian, Thin Solid Films 2018, 659, 48.

[111] B.-J. Li, Y.-Y. Wang, L.-J. Huang, H.-D. Cao, Q. Wang, N.-F. Ren, H. Ding, Ceram. Int. 2018, 44, 22225.

[112] R. Molaei, R. Bayati, J. Narayan, Cryst. Growth Des. 2013, 13, 5459.

[113] C. N. Chen, J. J. Huang, J. Appl. Res. Technol. 2015, 13, 170.

[114] T. Tsuchiya, K. Daoudi, A. Watanabe, T. Kumagai, S. Mizuta, J. Phys:. Conf. Ser. 2007, 59, 224.

[115] F. Z. Escola, N. Mingolo, O. E. Martínez, J. J. Rocca, C. S. Menoni, Opt. Express 2019, 27, 5729.

[116] S. Kuchipudi, I. N. Lin, H. C. Lee, Y. C. Lin, Y. L. Luo, J. H. Huang, N. H. Tai, S. J. Lin, Integr. Ferroelectr. 2003, 52, 119

[117] B. Li, H. Li, L. Huang, H. Cao, W. Zu, N. Ren, H. Ding, X. Kong, J. Zhang, Ceram. Int. 2017, 43, 7329.

[118] H. Pan, N. Misra, S. H. Ko, C. P. Grigoropoulos, N. Miller E. E. Haller, O. Dubon, Appl. Phys. A: Mater. Sci. Process. 2009, 94, 111.

[119] N. Kalfagiannis, D. C. Koutsogeorgis, E. Lidorikis, P. Patsalas, in Nanoplasmonics-Fundamentals and Applications, (Ed: G. Barbillon), InTechOpen, London, UK 2017, pp. 433-459.

[120] R. A. Ganeev, Laser-Surface Interactions, SpringerNetherlands, Dordrecht 2014 
[121] E. Carpene, D. Höche, P. Schaaf, in Laser Processing of Materials, vol 139 (Ed: P. Schaaf), Springer, Berlin 2010, pp. 21-47.

[122] K. Kim, S. Kim, S. Y. Lee, Curr. Appl. Phys. 2012, 12, 585.

[123] G. Fiaschi, S. Mirabella, G. Franzò, L. Maiolo, A. Chitu, Y. Komem, Y. Shacham-Diamand, Appl. Surf. Sci. 2018, 458, 800.

[124] H. Lu, Y. Tu, X. Lin, B. Fang, D. Luo, A. Laaksonen, Mater. Lett. 2010, 64, 2072.

[125] Q. Nian, M. Callahan, M. Saei, D. Look, H. Efstathiadis, J. Bailey, G. J. Cheng, Sci. Rep. 2015, 5, 15517.

[126] H. J. Kim, M. J. Maeng, J. H. Park, M. G. Kang, C. Y. Kang, Y. Park, Y. J. Chang, Curr. Appl. Phys. 2019, 19, 168.

[127] T. Park, D. Kim, Thin Solid Films 2015, 578, 76

[128] A. A. Serkov, H. V. Snelling, S. Heusing, T. M. Amaral, Sci. Rep. 2019, 9, 1773

[129] C. W. Cheng, W. C. Shen, C. Y. Lin, Y. J. Lee, J. S. Chen, Appl. Phys. A: Mater. Sci. Process. 2010, 101, 243.

[130] C. Chen, G. Chen, H. Yang, G. Zhang, D. Hu, H. Chen, T. Guo, J. Mater. Chem. C 2017, 5, 9273.

[131] L. Museur, J.-P. Michel, P. Portes, A. Englezis, A. Stassinopoulos, D. Anglos, A. V. Kanaev, J. Opt. Soc. Am. B 2010, 27, 531.

[132] E. V. Ramana, N. M. Ferreira, A. Mahajan, M. C. Ferro, F. Figueiras, M. P. F. Graça, M. A. Valente, J. Phys. Chem. Solids 2018, 113, 94.

[133] J. Kim, J. H. Ji, S. W. Min, G. H. Jo, M. W. Jung, M. J. Park, S. K. Lee, J. H. Koh, Ceram. Int. 2017, 43, 3900.

[134] C.-Y. Chung, B. Zhu, D. G. Ast, R. G. Greene, M. O. Thompson, Appl. Phys. Lett. 2015, 106, 123506

[135] H. Huang, H. Hu, J. Zhu, T. Guo, J. Electron. Mater. 2017, 46, 4497

[136] H. Palneedi, D. Maurya, G. Y. Kim, S. Priya, S. J. L. Kang K. H. Kim, S. Y. Choi, J. Ryu, Appl. Phys. Lett. 2015, 107, 012904.

[137] H. Palneedi, D. Maurya, G.-Y. Kim, V. Annapureddy, M.-S. Noh, C.-Y. Kang, J.-W. Kim, J.-J. Choi, S.-Y. Choi, S.-Y. Chung, S.-J. L. Kang, S. Priya, J. Ryu, Adv. Mater. 2017, 29, 1605688.

[138] G. H. Jo, J. H. Koh, Ceram. Int. 2019, 45, 6190.

[139] M. Lluscà, J. López-Vidrier, S. Lauzurica, M. I. Sánchez-Aniorte, A. Antony, C. Molpeceres, S. Hernández, B. Garrido, J. Bertomeu, J. Lumin. 2015, 167, 101

[140] G. Jo, J.-H. Ji, K. Masao, J.-G. Ha, S.-K. Lee, J.-H. Koh, Ceram. Int. 2018, 44, S211.

[141] D. H. Triyoso, G. Spencer, R. I. Hegde, R. Gregory, X.-D. Wang, Appl. Phys. Lett. 2008, 92, 113501

[142] C. Wei, D. Deng, G. Tian, H. He, J. Shao, Z. Fan, Optik 2008, 119,624

[143] X. Tian, Y. Li, Z. Xu, Thin Solid Films 2009, 517, 5855.

[144] C. K. Chung, K. P. Chuang, S. Y. Cheng, S. L. Lin, K. Y. Hsie, J. Alloys Compd. 2013, 574, 83.

[145] D. H. Kim, J. W. Park, Y. M. Chang, D. Lim, H. Chung, Thin Solid Films 2010, 518, 2812

[146] N. Kalfagiannis, A. Siozios, D. V. Bellas, D. Toliopoulos, L. Bowen, N. Pliatsikas, W. M. Cranton, C. Kosmidis, D. C. Koutsogeorgis, E. Lidorikis, P. Patsalas, Nanoscale 2016, 8, 8236.

[147] A. Siozios, N. Kalfagiannis, D. V Bellas, C. Bazioti, G. P. Dimitrakopulos, G. Vourlias, W. M. Cranton, E. Lidorikis, D. C. Koutsogeorgis, P. Patsalas, Nanotechnology 2015, 26, 155301

[148] M. S. Brown, C. B. Arnold, in Laser Precision Microfabrication, vol. 135 (Eds: K. Sugioka, M. Meunier, A. Piqué), Springer, Berlin, Heidelberg 2010, pp. 91-120.

[149] H. Palneedi, J. H. Park, D. Maurya, M. Peddigari, G.-T. Hwang, V. Annapureddy, J.-W. Kim, J.-J. Choi, B.-D. Hahn, S. Priya, K. J. Lee, J. Ryu, Adv. Mater. 2018, 30, 1705148

[150] A. H. Hamad, in High Energy and Short Pulse Lasers, (Ed: R. Viskup), InTech, Rijeka 2016, pp. 305-325.

[151] T. Zhu, Y. P. Wang, L. Zhou, Z. G. Liu, Mater. Sci. Eng. B, 2002, 89, 390-393.

[152] Z. Zhang, J. Zhu, D. Su, J. Liu, H. Shen, Y. Wang, L. Kang, J. Zhou, S. Yang, P. Wu, Thin Solid Films 2000, 375, 172.
[153] V. S. Teodorescu, A. V. Maraloiu, M. G. Blanchin, T. Yamada, C. S. Sandu, P. Delaporte, M. Zaharescu, in CAS 2013, Int. Semiconductor Conf., IEEE, Piscataway, USA 2013, pp. 77-80.

[154] J. L. Wang, Y. S. Lai, B. S. Chiou, D. C. Shye, C. C. Hwang, H. C. Cheng, J. Cryst. Growth 2007, 306, 80.

[155] P. Darmawan, P. S. Lee, Y. Setiawan, J. Ma, T. Osipowicz, Appl. Phys. Lett. 2007, 91, 092903.

[156] S. S. N. Bharadwaja, J. Kulik, R. Akarapu, H. Beratan, S. Trolier-McKinstry, IEEE Trans. Ultrason. Ferroelectr. Freq. Control 2010, 57, 2182.

[157] M. G. Kang, K. H. Cho, S. M. Oh, Y. H. Do, C. Y. Kang, S. Kim, S. J. Yoon, Curr. Appl. Phys. 2011, 11, S66.

[158] R. Bayati, R. Molaei, A. Richmond, S. Nori, F. Wu, D. Kumar, J. Narayan, J. G. Reynolds, C. L. Reynolds, ACS Appl. Mater. Interfaces 2014, 6, 22316

[159] X. Pan, Y. Shuai, C. Wu, W. Luo, X. Sun, Y. Yuan, S. Zhou, X. Ou W. Zhang, Appl. Surf. Sci. 2016, 389, 1104.

[160] M.-G. Kang, K.-H. Cho, Y. Ho Do, Y.-J. Lee, S. Nahm, S.-J. Yoon, C.-Y. Kang, Appl. Phys. Lett. 2012, 101, 242910.

[161] O. O. Gavrylyuk, O. Y. Semchuk, O. V. Steblova, A. A. Evtukh, L. L. Fedorenko, O. L. Bratus, S. O. Zlobin, M. Karlsteen, Appl. Surf. Sci. 2015, 336, 217.

[162] B. J. Li, L. J. Huang, N. F. Ren, X. Kong, Y. L. Cai, J. L. Zhang, Appl. Surf. Sci. 2015, 351, 113.

[163] B. jia Li, Y. ying Wang, L. jing Huang, H. di Cao, Q. Wang, H. Ding, N. fei Ren, Appl. Surf. Sci. 2018, 458, 940.

[164] M.-F. Chen, K. Lin, Y.-S. Ho, Opt. Lasers Eng. 2012, 50, 491.

[165] M. Noh, I. Seo, J. Park, J.-S. Chung, Y. S. Lee, H. J. Kim, Y. J. Chang, J.-H. Park, M. G. Kang, C. Y. Kang, Curr. Appl. Phys. 2016, 16, 145.

[166] S. O. El Hamali, W. M. Cranton, N. Kalfagiannis, X. Hou, R. Ranson, D. C. Koutsogeorgis, Opt. Lasers Eng. 2016, 80, 45.

[167] W. M. Cranton, S. L. Wilson, R. Ranson, D. C. Koutsogeorgis, K. Chi, R. Hedgley, J. Scott, S. Lipiec, A. Spiller, S. Speakman, Thin Solid Films 2007, 515, 8534.

[168] T. Tsuchiya, A. Watanabe, T. Kumagai, S. Mizuta, Appl. Surf. Sci. 2005, 248, 118

[169] T. Tsuchiya, F. Yamaguchi, I. Morimoto, T. Nakajima, T. Kumagai, Appl. Phys. A: Mater. Sci. Process. 2010, 99, 745.

[170] H. Pan, D. Lee, S. H. Ko, C. P. Grigoropoulos, H. K. Park, T. Hoult, Appl. Phys. A 2011, 104, 29.

[171] G. H. Jo, S. H. Kim, J. H. Koh, Ceram. Int. 2018, 44, 735

[172] N. F. Ren, L. J. Huang, B. J. Li, M. Zhou, Appl. Surf. Sci. 2014, 314, 208.

[173] N. Nedyalkov, M. Koleva, R. Nikov, P. Atanasov, Y. Nakajima, A. Takami, A. Shibata, M. Terakawa, Appl. Surf. Sci. 2016, 374, 172.

[174] C. Tsakonas, W. Cranton, F. Li, K. Abusabee, A. Flewitt, D. Koutsogeorgis, R. Ranson, J. Phys. D: Appl. Phys. 2013, 46, 095305.

[175] R. Vinodkumar, V. Jeena, V. Jiji, S. K. Saji, N. V. Unnikrishnan, Optik 2018, 174, 274

[176] Y. Li, O. R. Musaev, J. M. Wrobel, M. B. Kruger, Appl. Phys. A 2018, 124, 499.

[177] M. E. Koleva, N. N. Nedyalkov, N. Fukata, W. Jevasuwan, S. Amoruso, T. Koutzarova, G. V. Avdeev, B. Georgieva, D. Karashanova, Superlattices Microstruct. 2017, 109, 886.

[178] J. K. Yao, F. Ye, P. Fan, Opt. Mater. Express 2019, 9, 2545.

[179] E. H. Khan, S. C. Langford, J. T. Dickinson, L. A. Boatner, W. P. Hess, Langmuir 2009, 25, 1930.

[180] Y. Zhao, Y. Jiang, J. Appl. Phys. 2008, 103, 114903.

[181] D. Shiojiri, R. Yamauchi, D. Fukuda, N. Tsuchimine, S. Kaneko, A. Matsuda, M. Yoshimoto, J. Cryst. Growth 2015, 424, 38.

[182] C.-Y. Tsay, T.-T. Huang, Mater. Chem. Phys. 2013, 140, 365.

[183] M. Nakata, K. Takechi, K. Azuma, E. Tokumitsu, H. Yamaguchi, S. Kaneko, Appl. Phys. Express 2009, 2, 021102.

[184] M. Nakata, K. Takechi, S. Yamaguchi, E. Tokumitsu, H. Yamaguchi, S. Kaneko, Jpn. J. Appl. Phys. 2009, 48, 115505. 
[185] M. Nakata, K. Takechi, T. Eguchi, E. Tokumitsu, H. Yamaguchi, S. Kaneko, Jpn. J. Appl. Phys. 2009, 48, 0816071.

[186] Y.-H. Yang, S. S. Yang, K.-S. Chou, IEEE Electron Device Lett. 2010, 31, 969.

[187] C. H. Wu, H. Y. Huang, S. J. Wang, K. M. Chang, IEEE Electron Device Lett. 2014, 35, 1031

[188] C.-H. Wu, K.-M. Chang, Y.-M. Chen, B.-W. Huang, Y.-X. Zhang, S.-J. Wang, J. Nanosci. Nanotechnol. 2018, 18, 1917.

[189] J.-J. Kim, J.-Y. Bak, J.-H. Lee, H. S. Kim, N.-W. Jang, Y. Yun, W.-J. Lee, Thin Solid Films 2010, 518, 3022.

[190] J. Maeng, S. Heo, G. Jo, M. Choe, S. Kim, H. Hwang, T. Lee, Nanotechnology 2009, 20, 095203.

[191] M.-S. Oh, R. Nirmala, R. Navamathavan, J. Electron. Mater. 2019, $48,3137$.

[192] M. Nakata, H. Tsuji, Y. Fujisaki, H. Sato, Y. Nakajima, T. Takei, T. Yamamoto, T. Kurita, Appl. Phys. Lett. 2013, 103, 142111.

[193] M. Nakata, H. Tsuji, Y. Fujisaki, Y. Nakajima, T. Takei, T. Yamamoto, IEEE Trans. Ind. Appl. 2017, 53, 5972.

[194] J. P. Bermundo, Y. Ishikawa, M. N. Fujii, T. Nonaka, R. Ishihara, H. Ikenoue, Y. Uraoka, J. Phys. D: Appl. Phys. 2016, 49, 035102.

[195] J. P. S. Bermundo, Y. Ishikawa, M. N. Fujii, H. Ikenoue, Y. Uraoka, Appl. Phys. Lett. 2017, 110, 133503.

[196] J. P. S. Bermundo, C. Kulchaisit, D. C. Corsino, A. Syairah, M. N. Fujii, H. Ikenoue, Y. Ishikawa, Y. Uraoka, SID Symp. Dig. Tech. Pap. 2019, 50, 422.

[197] J. Lee, S. Song, S. Cho, M. Song, Y. Kim, J. Kwon, M. Han, in 19th International Workshop on Active-Matrix Flatpanel Displays and Devices (AM-FPD), IEEE, Piscataway, USA 2012, pp. 135-138.

[198] M. Fujii, Y. Ishikawa, R. Ishihara, J. van der Cingel, M. R. T. Mofrad, M. Horita, Y. Uraoka, Appl. Phys. Lett. 2013, 102, 122107.

[199] H. E. Edgerton, K. J. Germeshausen, US patent no. 2,269,338A, 1939.

[200] G. A. Kachurin, E. V. Nidaev, Sov. Phys. Semicond. 1977, 11, 1178.

[201] R. L. Cohen, J. S. Williams, L. C. Feldman, K. W. West, Appl. Phys. Lett. 1978, 33, 751

[202] L. Correra, L. Pedulli, Appl. Phys. Lett. 1980, 37, 55.

[203] A. Usami, Y. Tokuda, M. Katayama, S. Kaneshima, T. Wada, J. Phys. D: Appl. Phys. 1986, 19, 1079.

[204] B. Loisel, B. Guenais, A. Poudoulec, P. Henoc, Thin Solid Films 1984, 117, 117.

[205] B. Pécz, L. Dobos, D. Panknin, W. Skorupa, C. Lioutas, N. Vouroutzis, Appl. Surf. Sci. 2005, 242, 185.

[206] K. Ohdaira, Y. Endo, T. Fujiwara, S. Nishizaki, H. Matsumura, Jpn. J. Appl. Phys. 2007, 46, 7603.

[207] J. Murota, M. Sakuraba, S. Ono, Appl. Phys. Lett. 1993, 62, 2353.

[208] D. Panknin, J. Stoemenos, M. Eickhoff, V. Heera, M. Voelskow, W. Skorupa, Appl. Surf. Sci. 2001, 184, 377.

[209] K. A. Schroder, S. C. McCool, W. F. Furlan, TechConnect Briefs, 2006, 3, 198.

[210] J. S. Kang, J. Ryu, H. S. Kim, H. T. Hahn, J. Electron. Mater. 2011, 40, 2268.

[211] Y. Galagan, E. W. C. Coenen, R. Abbel, T. J. Van Lammeren, S. Sabik, M. Barink, E. R. Meinders, R. Andriessen, P. W. M. Blom, Org. Electron. 2013, 14, 38.

[212] S. Norita, D. Kumaki, Y. Kobayashi, T. Sato, K. Fukuda, S. Tokito, Org. Electron. 2015, 25, 131.

[213] S. H. Park, H. S. Kim, Thin Solid Films 2014, 550, 575.

[214] J. Ouyang, D. Cormier, S. A. Williams, D. A. Borkholder, J. Am. Ceram. Soc. 2016, 99, 2569.

[215] J. Ouyang, D. Cormier, D. A. Borkholder, ACS Appl. Energy Mater. 2019, 2, 338.

[216] D. Angmo, T. T. Larsen-Olsen, M. Jørgensen, R. R. Søndergaard, F. C. Krebs, Adv. Energy Mater. 2013, 3, 172.

[217] H. Kang, E. Sowade, R. R. Baumann, ACS Appl. Mater. Interfaces 2014, 6, 1682
[218] L. Rebohle, S. Prucnal, W. Skorupa, Semicond. Sci. Technol. 2016, 31, 103001.

[219] H. Kiyama, T. Kusuda, S. Kato, T. Aoyama, in IWJT-2008-Ext. Abstr. 2008 Int. Work. Junction Technol.Shangai, China, 2008, 1, 206.

[220] M. Voelskow, R. A. Yankov, W. Skorupa, in Subsecond Annealing of Advanced Materials, Vol. 192 (Eds: W. Skorupa, H. Schmidt), Springer, Cham 2014, pp. 1-13.

[221] K. A. Schroder, TechConnect Briefs 2011, 2, 220.

[222] D. Reichel, W. Skorupa, W. Lerch, J. C. Gelpey, Crit. Rev. Solid State Mater. Sci. 2011, 36, 102.

[223] T. Kubo, T. Sukegawa, E. Takii, T. Yamamoto, S. Satoh, M. Kase, in 2007 15th Int. Conf. on Advanced Thermal Processing of Semiconductors, IEEE, Piscataway, USA 2007

[224] C. Kang, H. Kim, Y. Oh, K. Baek, L. Do, IEEE Electron Device Lett. 2016, 37, 595.

[225] T. Druffel, R. Dharmadasa, B. W. Lavery, K. Ankireddy, Sol. Energy Mater. Sol. Cells 2018, 174, 359.

[226] G. G. Bentini, L. Correra, J. Appl. Phys. 1983, 54, 2057.

[227] N. M. Twyman, K. Tetzner, T. D. Anthopoulos, D. J. Payne, A. Regoutz, Appl. Surf. Sci. 2019, 479, 974.

[228] D. W. Kim, J. Park, J. Hwang, H. D. Kim, J. H. Ryu, K. B. Lee, K. H. Baek, L.-M. Do, J. S. Choi, Electron. Mater. Lett. 2015, 11, 82.

[229] K. Tetzner, Y.-H. Lin, A. Regoutz, A. Seitkhan, D. J. Payne, T. D. Anthopoulos, J. Mater. Chem. C 2017, 5, 11724.

[230] A. Kanjilal, I. Tyagulskii, D. Grambole, S. Tyagulskii, M. Voelskow, J. Lehmann, L. Rebohle, W. Skorupa, A. Nazarov, M. Helm, S. Prucnal, J. Appl. Phys. 2009, 106, 123103.

[231] K. Tetzner, K. A. Schroder, K. Bock, Ceram. Int. 2014, 40, 15753.

[232] L. Zhang, R. Tu, D. B. Chrisey, K. A. Schroder, S. Adireddy, J. He, Y. Zhang, B. B. Bourgeois, S. Luo, S. Farnsworth, W. Liu, B. C. Riggs, V. Puli, K. Sun, M. M. Oguntoye, S. Zhang, J. T. Shipman, J. Mater. Res. 2017, 32, 1701.

[233] E. Hensel, K. Wollschläger, D. Schulze, U. Kreissig, W. Skorupa, J. Finster, Surf. Interface Anal. 1985, 7, 207.

[234] T. Gebel, L. Rebohle, R. Fendler, W. Hentsch, W. Skorupa, M. Voelskow, W. Anwand, R. A. Yankov, in 14th International Conference on Advanced Thermal Processing of Semiconductors, Kyoto, Japan, 2006, pp. 47-55.

[235] T. Gebel, M. Neubert, R. Endler, J. Weber, M. Vinnichenko, A. Kolitsch, W. Skorupa, H. Liepack, in Mater. Res. Soc. Symp. Proc., 2011, 1287.

[236] J. H. Noh, P. C. Joshi, T. Kuruganti, P. D. Rack, IEEE J. Electron Devices Soc. 2015, 3, 297.

[237] S. J. Moon, K. M. Yu, S. H. Jeong, J. Y. Kim, B. K. Kim, H. J. Kim, E. J. Yun, B. S. Bae, ECS Trans. 2014, 64, 109.

[238] W. H. Lee, S. J. Lee, J. A. Lim, J. H. Cho, RSC Adv. 2015, 5, 78655.

[239] S. C. Park, D. Kim, H. Shin, D. K. Lee, X. Zhang, J. Park, J. S. Choi, J. Inf. Disp. 2016, 17, 1.

[240] M. Benwadih, R. Coppard, K. Bonrad, A. Klyszcz, D. Vuillaume, ACS Appl. Mater. Interfaces 2016, 8, 34513.

[241] S. K. Garlapati, J. S. Gebauer, S. Dehm, M. Bruns, M. Winterer, H. Hahn, S. Dasgupta, Adv. Electron. Mater. 2017, 3, 1600476.

[242] T.-Y. Eom, C.-H. Ahn, J.-G. Kang, M. S. Salman, S.-Y. Lee, Y.-H. Kim, H.-J. Lee, C.-M. Kang, C. Kang, Appl. Phys. Express 2018, 11, 061104.

[243] H. J. Jeong, H. M. Lee, C. H. Ryu, E. J. Park, K. L. Han, H. J. Hwang, K. C. Ok, H. S. Kim, J. S. Park, ACS Appl. Mater. Interfaces 2019, 11, 4152.

[244] C. J. Moon, H. S. Kim, ACS Appl. Mater. Interfaces 2019, 11, 13380.

[245] S. Kamiyama, T. Miura, Y. Nara, Electrochem. Solid-State Lett. 2006, 8, G367.

[246] J. Lehmann, R. Hübner, J. V. Borany, W. Skorupa, T. Mikolajick, A. Schäfer, J. Schubert, S. Mantl, Microelectron. Eng. 2013, 109, 381.

[247] J. V. Borany, J. Schubert, S. Mantl, J. M. J. Lopez, N. Shevchenko, W. Skorupa, J. Lehmann, A. Mücklich, Microelectron. Eng. 2011, 88, 1346.

[248] R. Delmdahl, R. Pätzel, J. Phys. D: Appl. Phys. 2014, 47, 034004. 